University of Rhode Island

DigitalCommons@URI

Open Access Dissertations

2015

\title{
Understanding the Barrier and Facilitators of Lifestyle Intervention Programs for Preventing Diabetes in High Risk Hispanic Adults
}

Lisa Adorno DiMaria

University of Rhode Island, L.dimaria@verizon.net

Follow this and additional works at: https://digitalcommons.uri.edu/oa_diss

\section{Recommended Citation}

DiMaria, Lisa Adorno, "Understanding the Barrier and Facilitators of Lifestyle Intervention Programs for Preventing Diabetes in High Risk Hispanic Adults" (2015). Open Access Dissertations. Paper 404. https://digitalcommons.uri.edu/oa_diss/404

This Dissertation is brought to you for free and open access by DigitalCommons@URI. It has been accepted for inclusion in Open Access Dissertations by an authorized administrator of DigitalCommons@URI. For more information, please contact digitalcommons-group@uri.edu. 


\section{UNDERSTANDING THE BARRIERS AND FACILITATORS OF LIFESTYLE INTERVENTION PROGRAMS FOR PREVENTING DIABETES IN HIGH RISK HISPANIC ADULTS \\ BY LISA ADORNO DIMARIA}

A DISSERTATION SUBMITTED IN PARTIAL FULFILLMENT OF THE REQUIREMENTS FOR THE DEGREE OF DOCTOR OF PHILOSOPHY

IN

NURSING

UNIVERSITY OF RHODE ISLAND 


\section{DOCTOR OF PHILOSOPHY IN NURSING DISSERTATION \\ $\mathrm{OF}$ \\ LISA ADORNO DIMARIA}

\section{APPROVED:}

Dissertation Committee:

Major Professor Patricia Burbank

Diane C. Martins

Alison Tovar

Nasser H. Zawia

DEAN OF THE GRADUATE SCHOOL

UNIVERSITY OF RHODE ISLAND

2015 


\section{ABSTRACT}

Since the Diabetes Prevention Program Research Group (2002) concluded that type 2 diabetes [T2D] can be prevented or delayed in pre-diabetic individuals, translation of the study's intensive lifestyle intervention has been a research priority. Translational research conducted with the high-risk Hispanic population is in its infancy. In general, the needs of this population for the development and refinement of interventions to prevent T2D have been neglected. This qualitative study explored intervention components and the perceived facilitators and barriers to making and sustaining dietary and physical activity behavior changes within the context of a lifestyle intervention program designed for a high-risk Hispanic population. This study also explored the impact of the lifestyle intervention program and individual behavior change on the lives of participants. Three focus group interviews and one individual interview were conducted with 17 uninsured, predominantly Spanish-speaking individuals who completed a lifestyle program offered by a free urban community health clinic in the northeast region of the U.S. A moderator's guide with semistructured interview questions was used. Interviews were translated and transcribed verbatim. Data analysis was conducted using an inductive data-driven approach. Krueger and Casey's (2000) criteria for coding and Sandelowski's (1995) suggestions for qualitative data analysis provided guidance for analysis. Participants described the program they attended as (1) a novel learning experience that provided practical and detailed information, (2) a supportive environment that was motivating and encouraging and (3) a catalyst for changing dietary and physical activity behaviors. Participants voiced the desire to both maintain changes and have access to more program sessions with opportunities for physical activity. Participants described 
rapidly adopting multiple dietary and physical activity behaviors by linking health behavior to health outcomes and self-regulating through cognitive processes. Three facilitators of dietary and physical activity behaviors were identified: (1) physical and emotional benefits, (2) social support and (3) persistence. Barriers identified were physical sensations, social and emotional aspects of everyday life and unawareness of healthy food options. Program impact included a preference for life after making behavior changes, an increased consciousness that helped sustain behaviors and the desire to continue forward. Participants also recognized the effect their individual changes had on others. In summary, this study found that education, in-person support and program duration were important components of a lifestyle intervention program for a high-risk Hispanic population. Further research is needed to explore tailoring of evidence-based lifestyle interventions and issues related to the sustainability of programs aimed at reducing T2D in high-risk populations. 


\section{ACKNOWLEDGMENTS}

I would like to express my extreme gratitude to the individuals who agreed to share their time and stories with me for this research. My intent was to not only fill a much needed gap, but to honor their words as well as their worlds. Special thanks to Dr. Annie DeGroot and Valerie Joseph for welcoming me into their clinic and entrusting me to explore a program that is near to their hearts. I am indebted to the community health workers, Damaris Rosales, Ingrid Castillo, Rosa Roman and Petra Cintron and nursing students, Esthefany Cabrera and Dilennys Perez who assisted me; without them, this research would not have been possible. Tu generosida no será olvidado.

I was once told by a wise professor that writing a dissertation is lonely work. Indeed, she was correct. When I listened closely enough though, I could hear the voices of my biggest supporters urging me on. In my mind's eye, I could see encouraging words written on small handwritten notes from years gone by. I was very lucky to have these people with me and need to thank them for being there.

I have had no better role model than my mother. Mom, memories of you writing care plans and studying from Lippincott at the kitchen table had more of an impact than we both realized. I am so proud of everything you have accomplished. Thank you for your unwavering support.

Dad, thank you for your encouragement and always believing in me. To Bill, who sadly didn't get to see me cross the finish line, I thank for taking such wonderful care of me and for teaching me that life isn't a sprint, it's a marathon, to get a letter, you 
have to write a letter, two wrongs don't make a right, and that I sure know how to make a short story long.

My children are my true teachers in life. Em, I can only describe you as amazing. You are blessed with wisdom beyond your years; a wisdom I dare admit has been imparted to me rather than the other way around. I will never regret the (many) days I played hooky so that we could hang out. You are my best pal and I love you.

Aidan, you have taught me many lessons. I am so grateful for your unique way of experiencing the world and how it has pushed me to expand my own ways of thinking. Thank you for the time when you told me to "go do my own work." I needed that. I love you googolplex, buddy.

I often describe my husband as the nicest guy I've ever met but he's more than that. Rich, I know you never expected that I would be in graduate school for the majority of our married life and yet, it is true. There are not enough words to express my gratitude for your support. I look forward to many days ahead trying to find them.

To my earthly angels, the women in my life who have listened to and nurtured me, I thank you. I am so grateful for your friendship. Much love to you all.

Fate has placed in me in the midst of many talented people, particularly in my military career, who mentored and encouraged me and often gave a shove in matters of personal growth and education. Sadly, I have lost contact with too many. I acknowledge them here and intend to thank them by paying it forward in their honor.

I am indebted to the professors at the URI College of Nursing. Thank you to Dr. Pat Burbank who remained patient and supportive through several shifts in focus. Ever 
determined to do things in my own time, she accepted that without judgment. A million thank you's, Pat.

Dr. Diane Martins has been an incredible mentor and teacher. Thank you, Di, for being you: someone who sees the best in people, who is generous to a fault, and who is always willing to lend an ear or words of encouragement when most needed. I learned from a true pro.

I am very grateful to Dr. Donna Schwartz-Barcott who spent hours with me as I attempted to rein in my thoughts and organize them into a cohesive research project. Thank you, Donna, for your gentle guidance and support. A brainstorming session with you always left me energized with a renewed sense of where I was headed.

Special thanks to Dr. Judith Mercer and Dr. Deb Erickson-Owens for taking me, if only for a brief time, on their thrilling ride. Your dedication was and is inspiring. I will always hold a very special place in my heart for your work. Thank you for that amazing experience and allowing me to be part of a skillful research team.

I would like to thank Dr. Alison Tovar for her inspiration and guidance. Alison, I am fairly certain I would not have pursued this research had I not been fortunate enough to cross paths with you at just the right time. Thank you also to Dr. Rosa Maria Pegueros for agreeing to be part of my committee.

Lastly, I would be remiss to leave out my fellow peers who made the journey with me at the URI College of Nursing. I have had the extreme pleasure and privilege of going through this experience with the most knowledgeable and passionate nurses in the state. I thank them all for imparting their wisdom and sharing with me their most private stories related to nursing and life. 


\section{PREFACE}

"We shall not cease from exploration, and the end of all our exploring will be to arrive where we started and know the place for the first time." T.S. Eliot

I clearly recall the findings published by the Diabetes Prevention Program Research Group (2002) and its impact on my position as a health promotion director and public health nurse educator. Pre-diabetes and metabolic syndrome were relatively novel concepts to consider as colleagues and I developed healthy living and diabetes education programs for a military population. Since then, an unrelenting preoccupation with these concepts and their potential to progress to diabetes took hold without a clear vision for how it would best serve. Although a proponent of the evidence-based program, personal experiences with individual patients and groups of patients told me this was not a panacea. I was skeptical for two distinct reasons. Life for the military population I encountered was dynamic and often chaotic. I wondered how well the many behavioral concepts imbedded in the evidence based lifestyle intervention could be adopted by individuals and families faced with multiple challenges. Second, even before exposure to the philosophy of science, I questioned whether the model as presented could be applied universally. My nursing philosophy and practice, I thought, was built on meeting each patient without preconceptions; universals were dangerous and could potentially limit our interaction with one another. I've only now been able to articulate that the framework felt robotic and outside of the experiences of the patients I encountered. In addition, I recognize that I was operating under the assumption that a healthy lifestyle was an absolute possibility, though I was beginning to suspect that the means to get there were not universally similar. 
Reconciling these ideas with the prevailing research ideology became an academic journey. Akin to a curious traveler, I took detours and excursions to explore, seek understanding, and gain a true appreciation for each locale, some completely foreign. Metaphorically, I have never held a one-way non-stop ticket. The phenomenon of intervening to prevent diabetes was viewed from multiple lenses: philosophical, theoretical, and paradigmatic. Each held value yet seemingly, did not move me closer to a final destination. Discouraged, the topic was even completely abandoned for a time.

I had the fateful opportunity to observe a lifestyle intervention program designed for an uninsured Hispanic population in an urban community setting. At once, I arrived back at the place I had started. Again, I was questioning but with a distinctly different population confronted with seemingly discrete challenges. With clarity, the path that needed to be followed presented itself. Original pangs of skepticism have become what I now call research questions; questions that would have been meaningless without the journey. And, while I initially considered diabetes prevention my passion, I have also come to see it as the medium through which the real preoccupation was able to emerge: discovering what is true for the individuals, communities or populations who entrust my guidance to seek solutions in matters of health that are most important to them using methods best suited for them. 


\section{TABLE OF CONTENTS}

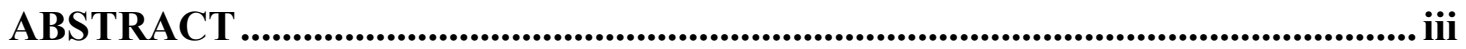

ACKNOWLEDGMENTS ............................................................................................. iv

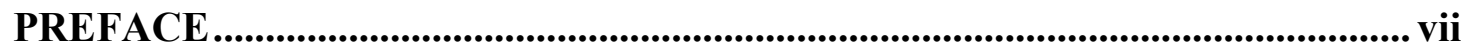

TABLE OF CONTENTS................................................................................................. ix

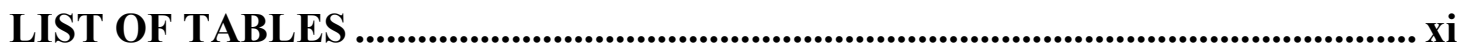

LIST OF ABBREVIATIONS .......................................................................

CHAPTER ONE INTRODUCTION _........................................................................... 1

Diabetes Risk and Relevance ...................................................................... 2

Diabetes Prevention with Intensive Lifestyle Intervention................................... 3

Significance of the Study to Nursing_............................................................ 5

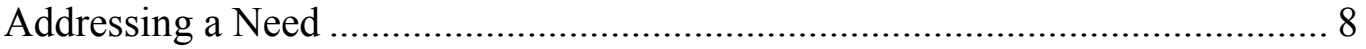

Purpose of Study and Design ......................................................................... 11

CHAPTER TWO REVIEW OF THE LITERATURE ............................................ 13

Definition of High Risk.................................................................................. 13

Historical Evolution \& Impact of Intensive Lifestyle Intervention ...................... 13

Translational Research in Community Settings .................................................. 22

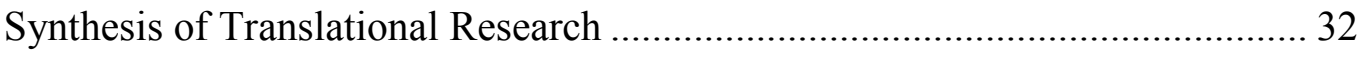

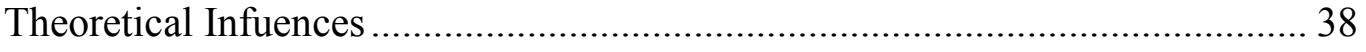

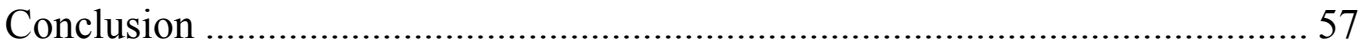

CHAPTER THREEE METHODOLOGY ............................................................. 62

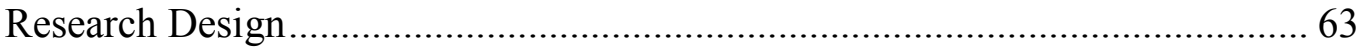




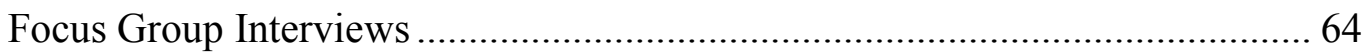

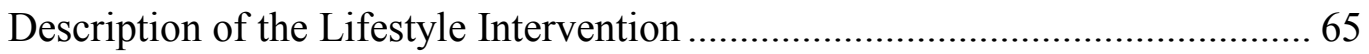

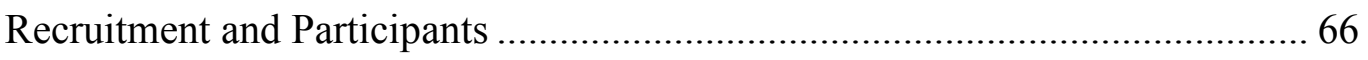

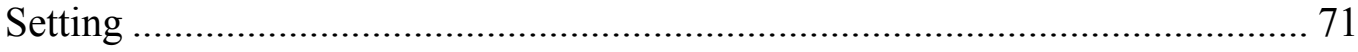

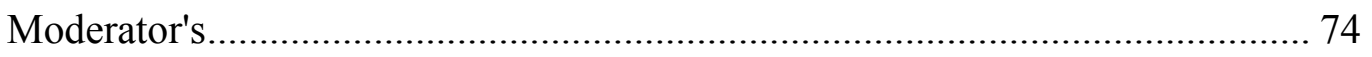

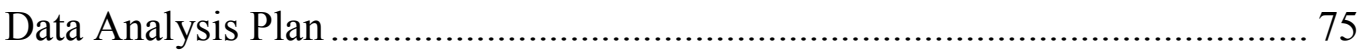

Ehtical Issues and Trustworthiness .......................................................... 76

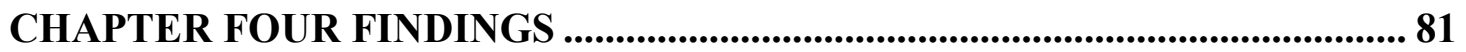

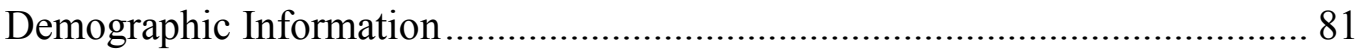

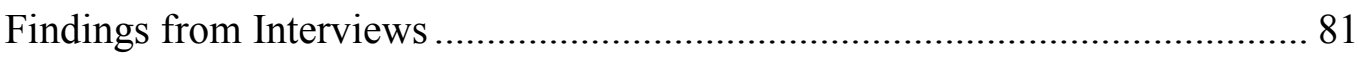

CHAPTER FIVE DISCUSSION AND CONCLUSION ................................... 124

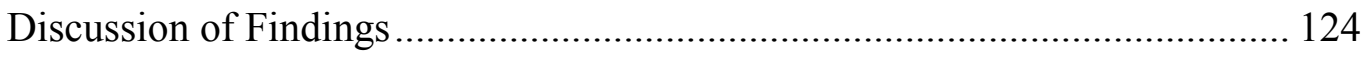

Theoretical Implications................................................................. 138

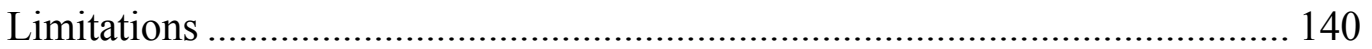

Implications for Nursing Research, Education and Practice......................... 144

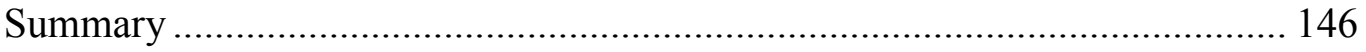

APPENDIX A Letter of Support..................................................................... 149

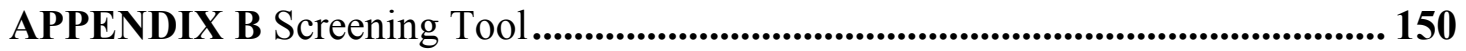

APPENDIX C Moderator's Guide .............................................................................. 151

APPENDIX D Informed Consent ....................................................................... 153

APPENDIX E Demographic Questionnaire ......................................................... 155

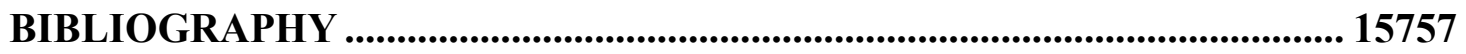




\section{LIST OF TABLES}

TABLE

PAGE

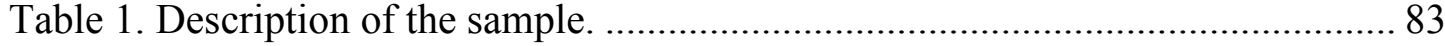

Table 2. Description of individual and focus group interviews................................... 84

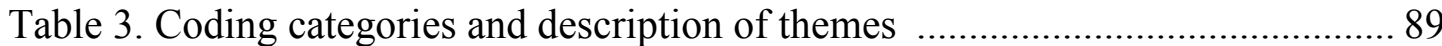




$\begin{array}{ll}\text { AADE } & \text { American Association of Diabetes Educators } \\ \text { ACA } & \text { Affordable Care Act } \\ \text { ADA } & \text { American Diabetes Association } \\ \text { AHRQ } & \text { Agency for Healthcare Research and Quality } \\ \text { AR } & \text { Action Research } \\ \text { CBPR } & \text { Community Based Participatory Research } \\ \text { CDC } & \text { Centers for Disease Control and Prevention } \\ \text { CHW } & \text { Community Health Worker } \\ \text { CVD } & \text { Cardiovascular } \\ \text { DEPLOY } & \text { Diabetes Education and Prevention with Lifestyle Intervention } \\ & \text { Offered at the YMCA } \\ \text { DPP } & \text { Diabetes Prevention Program } \\ \text { DPPRG } & \text { Diabetes Prevention Program Research Group } \\ \text { DPS } & \text { Diabetes Prevention Study } \\ \text { EBT } & \text { Evidence-based Treatment } \\ \text { GLB } & \text { Group Lifestyle Balance } \\ \text { HEED } & \text { Help Educate to Eliminate Diabetes } \\ \text { HELP PD } & \text { Healthy Living Partnerships to Prevent Diabetes } \\ \text { HLP } & \text { Healthy Living Program } \\ \text { IGT } & \text { Impaired Glucose Tolerance } \\ \text { ILI } & \text { Intensive Lifestyle Intervention } \\ \text { IOM } & \text { Institute of Medicine } \\ \text { LP } & \text { Lifestyle Intervention Program } \\ \text { LWBL } & \text { Live Well, Be Well } \\ \text { MI } & \text { Motivational Interviewing } \\ \text { NDPP } & \text { National Diabetes Prevention Program } \\ \text { PL-DPP } & \text { Promotora Led Diabetes Prevention Program } \\ \text { SCT } & \text { Social Cognitive Theory } \\ \text { SOC } & \text { Stage of Change } \\ \text { TTM } & \text { Transtheoretical Model } \\ \text { T2D } & \text { Type 2 Diabetes } \\ \text { WHO } & \text { World Health Organization } \\ \text { YMCA } & \text { Young Men's Christian Association } \\ & \end{array}$




\section{CHAPTER 1}

\section{INTRODUCTION}

It is well established that diabetes can be prevented or delayed in those at increased risk for the disease with intensive lifestyle intervention (Diabetes Prevention Program Research Group et al., 2009; Knowler et al., 2002). Since the landmark Diabetes Prevention Program (2002) clinical trial was published, an explosion of translational research has been conducted across practice settings. Significantly less research has been conducted with low income Hispanic populations in community settings. Interventions tailored to or adapted for this population provide conflicting evidence limiting what is known about how to best intervene to reduce the diabetes health disparity. Additionally, the long-term effectiveness of interventions has not been demonstrated, especially in high risk populations, leading to further speculation regarding the impact lifestyle interventions have on the daily lives of participants postintervention.

Diabetes in the U.S. has reached epidemic proportions (Shaw, Sicree \& Zimmet, 2010). There are currently 25.8 million people, or $8.3 \%$ of the total population, living with the disease (National Institute of Diabetes and Digestive and Kidney Disorders, 2011). Type 2 diabetes comprises the majority of these cases (American Diabetes Association, 2011). Type 2 diabetes is a chronic, progressive disease characterized by irreversible beta cell loss in the pancreas. Once diagnosed, the need for both medical management and self-management are complex and lifelong. The prevention of debilitating diabetes-related complications is dependent on optimal glycemic control; diabetes is the leading cause of kidney failure, non-traumatic lower limb amputations 
and new cases of blindness in the U.S. (Inzucchi et al., 2012).

The economic toll of diabetes is indisputable. The nation spends an estimated $\$ 176$ billion dollars on diabetes per year and is projected to double within the next 25 years (American Diabetes Association, 2011; American Diabetes Association, 2013; Huang, Basu, O'Grady, \& Capretta, 2009). Factoring in associated costs of undiagnosed cases, pre-diabetes and gestational diabetes, this jumps to a staggering \$218 billion (American Diabetes Association, 2011; American Diabetes Association, 2013; Dall et al., 2010).

\section{Diabetes Risk and Relevance}

Type 2 diabetes (T2D) develops insidiously over time rather than acutely and is preceded by a period of altered glucose metabolism referred to as pre-diabetes. This collective term refers to an elevation in post-prandial glucose, also called impaired glucose tolerance, an elevation in fasting glucose, referred to as impaired fasting glucose, or an elevated hemoglobin A1C. These can occur in isolation or in combination and, though elevations do not reach the diagnostic threshold for T2D, their presence significantly increases the risk of developing the disease. Thirty-nine percent of U.S. adults over the age of 20 are estimated to be pre-diabetic, with 5-10\% progressing to T2D per year (Nathan et al., 2007; Nichols, Hillier, \& Brown, 2007). The implications of this are dramatic when one considers the need for lifelong management, the associated costs and the potential for diabetes-related complications occurring at younger ages. Fifty percent of U.S. adults over the age of 60 have prediabetes (National Institute of Diabetes and Digestive and Kidney Disorders, 2011). This is especially salient with the projected growth in the aging population. By the 
year 2030, one in five U.S. adults will be over the age of 65 (US Census Bureau, 2010). T2D is now associated with cognitive decline, increased risk of falls and subsequent fractures and decreased mobility (Bruehl et al., 2009; Schneider et al., 2013). Insulin resistance, a hallmark feature of pre-diabetes and T2D, has also been associated with impaired cognitive function and increased risk of dementia and Alzheimer's disease (Bruehl, Sweat, Hassenstab, Polyakov, \& Convit, 2010; Craft, 2007; Crane et al., 2013).

The progression from pre-diabetes to T2D has economic and substantial quality of life implications. For these reasons, prevention is critical. In the pre-diabetic population, even a delay in onset of T2D must be viewed for its significance in preserving pancreatic beta cell function and maximizing quality of life.

\section{Diabetes Prevention through Lifestyle Intervention}

Research has demonstrated that progression from pre-diabetes to T2D is not inevitable (Norris et al., 2005; Perreault et al., 2009; Yoon, Kwok, \& Magkidis, 2013). Since the Diabetes Prevention Program Research Group [(DPPRG] (2002) concluded that $\mathrm{T} 2 \mathrm{D}$ can be prevented or delayed in pre-diabetic individuals, translation has been a research priority (Knowler et al., 2002). This multi-site national clinical trial found a $58 \%$ reduction in the incidence of $\mathrm{T} 2 \mathrm{D}$ in pre-diabetic subjects randomized to intensive lifestyle intervention (ILI). The study was the first diabetes prevention clinical trial to over-recruit members of U.S. racial and ethnic minority groups. The Diabetes Prevention Program (DPP) ILI was effective among all subgroups, though insufficiently powered to conduct subgroup analyses (Knowler et al., 2002).

The DPP has been an influential force in diabetes prevention. For example, the 
Centers for Disease Control and Prevention (CDC) implemented a National Diabetes Prevention Program (NDPP), providing funding to departments of health and professional organizations such as the American Association of Diabetes Educators (AADE) and the Young Men's Christian Association (YMCA) in select states. Through partnership with private insurance networks, the implementation of the NDPP is proposed to increase access to the program to those at higher risk for T2D (CDC, May 15, 2014). The NDPP is conceptually modeled after the DPP clinical trial and requires the use of the CDC NDPP curriculum, available in Spanish, and must be facilitated by a health care professional who has received the endorsed Lifestyle Coach training (CDC, May 15, 2014). Organizations who commit to this program incur the cost of training for staff members as well as the cost of applying for CDC recognition as an approved NDPP site. This program reflects considerable fidelity to the original ILI used in the DPP clinical trial.

It has been argued that, in order to realize clinical outcomes similar to the DPP, translational interventions should possess the multiple necessary components consistent with that conceptual model (Venditti \& Kramer, 2012; Whittemore, 2011). Evidence-based ILIs for diabetes prevention have been described as interventions that provide education and behavioral support over time, utilizing key intervention components to include specific weight loss and physical activity goals, ongoing behavioral support, a minimum dose and duration and the implementation of robust behavioral strategies (Venditti \& Kramer, 2012; Whittemore, 2011). Indeed, the literature reflects that the more modifications made to the evidence-based ILI in community settings, the less effective the ILI in achieving weight loss and physical 
activity goals (Ali, Echouffo-Tcheugui \& Williamson, 2012; Venditti \& Kramer, 2013). This illustrates a critical issue. While translational research often requires adapting or modifying interventions in order to make the leap from the clinical ideal to actual practice settings, determining the degree in which modifications can be made while yielding similar outcomes is problematic (Glasgow, 2003; Green \& Glasgow, 2006). This was particularly true in research conducted in high-risk Hispanic communities and in part explains the lack of research utilizing the evidence-based model in those communities.

The evidence-based model is a resource-intensive model. This affects translation but also sustainability. Low resource community settings such as community health centers and community organizations such as churches, senior centers, schools and shelters rely on public, private and academic funding to implement programs and must alter the evidence-based program out of necessity and practicality (Glasgow, 2003). The few experimental or quasi-experimental studies conducted in high-risk Hispanic communities were significantly modified, and were often unable to achieve the clinically meaningful weight loss needed to prevent diabetes; no studies have achieved physical activity goals (Kanaya et al., 2012; Ockene et al., 2012; Parikh et al., 2010; Ruggiero, Oros, \& Choi, 2011). This was despite adapting interventions for culture.

\section{Significance for Nursing}

In 2011, the Institute of Medicine [IOM] in partnership with the Robert Wood Johnson Foundation published the landmark report, The Future of Nursing: Leading Change, Advancing Health. Nursing is the largest segment of the U.S. health care system and, as such, will increasingly be called upon to take on new roles in both 
patient-care and leadership positions. The report advocated a transformation of the nursing profession in order to adequately meet the challenges of a rapidly changing health care system (IOM, 2011). Important recommendations were made in regards to nurses being allowed to fully practice within the scope of their education, attain higher levels of education, and become partners and leaders in the redesign of health care. Emphasis was placed on nurses utilizing evidence-based, patient-centered practices and improving the nation's health in less conventional practice settings. Nurses from diverse specialty areas such as public and community health and advanced practice nursing would fill roles in nurse-managed community clinics and community care collaboratives, especially in communities affected by health disparities. These would include primary care services, but as also mandated in the Affordable Care Act [ACA], provision of health promotion and disease prevention programs. This is already evident (IOM, 2011; Whittemore, Rosenberg, \& Jeon, 2013) and will continue as demand grows.

The prevention of chronic illnesses such as T2D is an enduring public health problem that continues to expand globally (Shaw, Sicree, \& Zimmet, 2010). Nurse researchers will be increasingly in the position to secure funding and conduct research with a focus on decreasing health and diabetes disparities. The IOM (2011) has stated, Nurse researchers must become active not only in studying important care delivery questions, but also in translating research findings into practice and developing and setting the policy agendas. Their leadership is vital in ensuring that new state and federal-level policies are based on evidence and will help increase quality and access while decreasing costs and health care 
disparities...Nurse researchers should seek funding from the National Institute for Nursing Research and other institutes of the National Institutes of Health, as do scientists from other disciplines, to help increase the evidence base for improved models of care.

The literature reveals a growing trend in academic and community research partnerships in high-risk communities. Nurse scientists, advanced practice nurses and nursing faculty are well positioned to create and sustain such partnerships in an attempt to bridge the research-to-practice gap. This is especially salient for diabetes prevention efforts which remain elusive and underrepresented by nursing in the literature. It is unclear to what extent ILIs can be modified, and which components are beneficial while remaining effective in high-risk Hispanic communities. This study contributes to the understanding of what is necessary or essential in ILIs for these communities. It will also increase the understanding of the perceived barriers or facilitators to lifestyle changes within the context of ILIs and will add clarity in relation to the impact ILIs have on the lives of participants. This serves to enhance future intervention development, which is significant for nursing as well as for all disciplines working to prevent T2D in low income Hispanic populations.

Kim (2010) proposed a typology of theoretical domains for nursing which include client, client-nurse, practice and environment which "force us to view reality from a nursing angle of vision" (p.59). The phenomenon of diabetes prevention in the highrisk Hispanic population was conceptualized from the client domain. Client is defined as an individual, a community or a population and was considered from Kim's (2000) Model of Human Living. According to Kim (2000), humans cannot be considered in 
isolation or "out of context” (p. 38). Kim's (2000) Model of Human Living

conceptualizes humans from three dimensions: living of oneself, living with others and living in situations. These three dimensions reflect the "integrative, dynamic intersection of biology, personhood and sociality" (Kim, 2000, p. 40), expanding the angle from which to view client phenomena, guide nursing responses and refine the role of nurse. Living of oneself refers to the uniqueness and subjectivity of the human experience which is biologic and social. Living with others refers to the social context such as the interactions with friends, family, community and society. Living in situations relates to social and environmental contexts which can be stable or dynamic and largely relates to how well humans adapt and respond to the environment (Kim, 2010).

\section{Addressing a Need}

While fidelity to evidence-based interventions is regarded as safe and effective, overreliance may obscure the ability to envision novel approaches to solve complex health problems. Evidence-based practice, particularly in nursing, integrates evidence provided by research as well as clinical expertise, patient expertise and local contextual factors (Rycroft-Malone et al., 2004). Over the course of one year, the researcher was a volunteer and member of an academic-community partnership at an urban free health clinic which provided health services for an uninsured, predominantly Hispanic population. The term Hispanic will be used throughout this dissertation. This is based on the personal preferences communicated to the researcher by the urban Hispanic population who participated in this research. The free clinic does not receive federal assistance and relies on grant funding and private and public 
donations to operate. The clinic is staffed by a medical director, nurse manager, office manager and bilingual community health workers. Additional staffing consists of volunteers of bilingual physicians, medical students, nursing students and volunteer medical assistants. In addition to having the opportunity to be part of the day-to-day routine at this clinic, the researcher was able to observe an evolving lifestyle intervention program over the course of six weeks with two separate groups of participants. The lifestyle intervention program, developed for an uninsured Hispanic urban community at high risk for T2D, was not conceptually influenced by the evidence-based model, yet was reportedly experiencing favorable preliminary clinical outcomes. Although it shared commonalities, factors or components considered essential in ILI to prevent T2D were absent. The curriculum, developed by a bilingual physician in Spanish, was grounded in a health education model with emphasis on improving health literacy, and was initially intended for use with individuals diagnosed with T2D. Program design was further informed by the social cognitive construct of observational learning. This lifestyle intervention program was facilitated primarily by community health workers of similar ethnic and socioeconomic backgrounds.

The lifestyle intervention program was noted to have several strengths and was also met with challenges. Recruitment was difficult. Sessions were well attended, but retention to the three-month follow up was poor. Group physical activity instruction was met positively; however, participants reported not being active on their own. These factors were consistent with the literature. The major inconsistency was that, despite its lack of congruence with the evidence-based program, participants were 
reporting weight loss, some clinically significant. Participants were observed to interact primarily with the program facilitator rather than one another. Successful weight loss was attributed by at least one participant to a belief in and assistance from a higher power. Another participant described the program as "life changing."

Based on the very limited data from the participant observation experience and the lack of exploration of lifestyle intervention programs from the perspective of the highrisk Hispanic population, it was unclear whether additional evidence-based components would be beneficial or, conversely, if other programmatic factors were at work that had yet to be identified. Unclear to the researcher was (1) which components of the evidence-based program were essential and (2) within those components, which concepts or factors potentially mediated clinical and behavioral outcomes for the highrisk Hispanic population in which little is known. The evidence-based model provides the best evidence to date for halting the progression to T2D. Few studies, however, have fully operationalized this framework with low income, uninsured urban Hispanic communities. In general, the needs of this population have been neglected in the development and refinement of interventions to prevent T2D.

The predominant theoretical approach to ILI for the prevention of T2D was the behaviorist perspective. Prominent theories consistent with this perspective include Social Cognitive Theory, the Transtheoretical Model and Motivational Interviewing. An ecological perspective was utilized under the assumption that environmental and social contexts influence health behavior. Lastly, a community-based participatory research (CBPR) approach was used, particularly with high-risk populations, to translate the evidence-based ILI. In contrast to the top down approach of the existing 
model, the CBPR approach engages members of communities or specific populations in multiple phases of the research process when translating the evidence-based ILI. This research was theoretically influenced by the social ecological and participatory perspectives with the rationale that: 1) individuals are more than their behavior; environmental factors have an influence on lifestyle behaviors and, 2) individuals and communities are experts in matters that relate to their own health.

\section{Purpose of the Study and Design}

The purpose of this research was to explore intervention components and to identify the perceived facilitators or barriers to making and sustaining lifestyle behavior changes within the context of a lifestyle intervention program to prevent T2D. The study also sought to explore the impact of the lifestyle intervention program and lifestyle behavior change in the lives of participants with the aim of gaining an understanding from the perspectives of a high-risk Hispanic population. The research questions were as follows:

1. How do participants describe in their own words, the lifestyle intervention program they attended?

2. What, how and to what extent were participants able to change physical activity and dietary behaviors during the eight week program and sustain those behaviors after the program?

3. What were the facilitators and barriers described by participants in changing physical activity and dietary behaviors and the ability to maintain those behaviors after the program?

4. What was the impact of the lifestyle intervention program and individual 
behavior changes on the lives of participants?

This qualitative research methodology used a descriptive exploratory design. Focus groups were viewed as a non-threatening means of data collection, with the less dominant role of the researcher potentially decreasing the perception of a power differential. The emphasis on participants in focus group interviews may also have empowered disenfranchised populations, allowing them to freely engage in discussion and reveal factors not previously considered (Halcomb, Gholizadeh, DiGiacomo, Phillips, \& Davidson, 2007; Krueger, 1994). Three focus group interviews and one individual interview were conducted with a homogenous sample comprised of participants who completed the lifestyle intervention program at the free health clinic previously described. The findings of this study will be presented and discussed, followed by the implications for future research and nursing practice. This work helps close an existing gap between the evidence based ILI and what is needed for diabetes prevention programs in high-risk Hispanic populations disproportionately affected by T2D. 


\section{CHAPTER 2}

\section{REVIEW OF LITERATURE}

More than a decade has passed since the landmark DPP (2002) clinical trial. Translation of this resource-intensive trial into real-world, community settings with high-risk populations is in its infancy. High-risk populations are disproportionately affected by T2D, yet they often do not benefit from what has become the gold standard. Intervening stands to increase quality of life and decrease the economic toll T2D takes on individuals, families and the health care system.

Critical to this research is whether different approaches are needed when intervening to prevent T2D in the high-risk Hispanic population disproportionately affected by T2D. This heterogeneous group now represents $16.7 \%$ of the U.S. population (Agardh, Allebeck, Hallqvist, Moradi, \& Sidorchuk, 2011), with an expected increase to $30 \%$ by the year 2050 (CDC, 2011). T2D is twice as prevalent in the Hispanic population and develops at a younger age (CDC, 2011). The prevalence of pre-diabetes is similar and remains constant regardless of country of origin (Hispanic Community Health Study/Study of Latinos Data Book: A Report to the Communities, 2013). Pre-diabetes often co-exists with the metabolic syndrome which is highly predictive of T2D (Grundy, 2012; Lorenzo et al., 2003; Lorenzo, Williams, Hunt, \& Haffner, 2007), and is more prevalent in the Hispanic population (Ford, Giles, \& Dietz, 2002). Metabolic syndrome is a cluster of risk factors which includes the presence of central adiposity, elevated fasting plasma glucose, dyslipidemia and hypertension. 


\section{Definition of Risk}

Pre-diabetes significantly increases the risk of developing T2D through insulin resistance and beta cell destruction, yet this physiologic definition provides a limited view of risk. T2D is one of several chronic diseases in which a disparity exists in incidence and prevalence (Agardh et al., 2011; Link \& McKinlay, 2009). The U.S. Hispanic population, diverse in itself, is affected by this disparity. A range of empirical and theoretical explanations for this have been proposed. Obesity is a known mediator and independent risk factor for T2D, and is more prevalent in the adult Hispanic population (Ogden \& Carroll, 2010; Oza-Frank \& Cunningham, 2010; Zhang, Wang, \& Huang, 2009). A sedentary lifestyle is also a risk factor for T2D. Studies consistently report low levels of leisure physical activity in the Hispanic population (Neighbors, Marques \& Marcus, 2008). This has been attributed not only to cultural beliefs and attitudes, but also to physical and social environments (D'Alonzo, 2012; Larsen, Noble, Murray, \& Marcus, 2014; Marquez \& McCauley, 2006). Obesity and physical activity levels have been linked to lower socioeconomic position as well as greater degree of acculturation (Perez-Escamilla, 2011). Even in the absence of obesity, the Hispanic population is at increased risk for T2D. Insulin resistance, one of the underlying pathologies in the development of T2D, has been proposed to be influenced by single nucleotide polymorphisms predisposing some Hispanics to decreased insulin sensitivity at normal or overweight (non-obese) status (Caballero, 2005).

Chaufan and Weitz (2009) argue that a focus on culture and ethnicity obscures the larger issue that influences health: poverty. Social determinants and health inequities 
are associated with the development of T2D. Twenty-six percent of the U.S. Hispanic populations live in poverty, almost three times that of non-Hispanic whites. Compared to $15.4 \%$ of adult non-Hispanic whites, $29.8 \%$ of the Hispanic population in the U.S. lack health insurance (R. A. Cohen \& Martinez, 2012), and 27\% report having no consistent place to receive care as compared to $16 \%$ of non-Hispanic blacks and $14 \%$ of non-Hispanic whites (Blackwell, Lucas, \& Clarke, 2014). Together with the African-American population, the Hispanic population is projected to experience the greatest gains in health insurance due to the ACA (Clemans-Cope, Kenney, Buettgens, Carroll, \& Blavin, 2012), yet more than half of the uninsured Hispanic population will remain uncovered. Those who reside in states that have opted out of the Medicaid expansion, earn incomes so low that coverage is not mandated, cannot afford the deductible for the lowest tier of coverage or are undocumented will not benefit (Clemans-Cope et al., 2012). Access to consistent quality health care is necessary for the treatment of chronic illness and primary and secondary prevention. Hispanic men and women are more likely to seek health care in acute circumstances (Ai, Appel, Huang, \& Lee, 2012; MacNaughton, 2008). This may be attributed to factors such as low health literacy which is correlated with poor health outcomes (Berkman, Sheridan, Donahue, Halpern, \& Crotty, 2011), lack of trust in providers and fear in seeking care. Even when insured, minority populations do not receive the same quality of health care (IOM, 2003).

Chronic stress related to social and health inequities is associated with increased T2D risk through biopsychosocial pathways (Mattei, Demissie, Falcon, Ordovas, \& Tucker, 2010; Seeman, Epel, Gruenewald, Karlamangla, \& McEwen, 2010). Repeated 
activation of the autonomic nervous system and hypothalamic pituitary axis causes dysregulation of the primary stress mediator's epinephrine, norepinephrine and cortisol. This is associated with insulin resistance and the development of abdominal adiposity (Mattei et al., 2010). Chronic stress also has effects on health behavior by way of evolutionary neural pathways (Dallman, 2010). The desire for certain foodsfor example, foods high in simple carbohydrates, fat and sodium — may have more to do with neurobiology than what is commonly attributed to a lack of self-control or motivation.

There are currently multiple empirical and theoretical explanations for the disparity in the development of T2D among Hispanic populations. In sum, they illustrate that risk has been conceptualized not only in terms of degree of risk exposure—-for example physiological processes, heritability, and health behaviorsbut also in terms of vulnerability (Frohlich \& Potvin, 2008). Social determinants and health inequities further exacerbate T2D risk. As a result, intervening with this highrisk population has become a national research priority (U.S. Department of Health and Human Services, Agency for Healthcare Research and Quality [AHRQ], 2013; IOM, 2009)

This chapter will explore the historical evolution of diabetes prevention through ILI and discuss its impact on how diabetes prevention is conceptualized today. The translational research will be described and synthesized in relation to intervention components and outcomes with particular emphasis on high-risk Hispanic populations in community settings. Community settings are defined, for the purpose of this research, as those that provide health related services to uninsured or vulnerable 
populations outside of traditional hospital or primary care settings (Geller, Taylor, \& Scott, 2004; IOM, 2003; U.S. Department of Health and Human Services, Agency for Healthcare Research and Quality , 2013). Examples are churches, public health departments, federally funded community health centers, non-profit community organizations and safety net clinics.

Translational ILI studies were included if they met the minimum defining criteria for ILI for the prevention of T2D: (1) the primary aim of ILI was to decrease weight or improve glycemia (2) the intervention targeted both physical activity and dietary behaviors and, (3) participants interacted with a facilitator. Studies that included participants with existing T2D in the ILI were excluded. The predominant theoretical influences will be discussed for their relevance and impact on the current state of ILI for T2D prevention with high-risk Hispanic populations. Lastly, a synthesis of the literature will be presented providing the rationale for this research. Databases searched for this review included PubMed, CINAHL, The Cochrane Library, PsychArticles, Sociological Abstracts, Project MUSE and the search engine Google Scholar.

\section{Historical Evolution and Impact of ILI}

Prior to 1970, studies that attempted to prevent or delay the onset of T2D were scarce. At that time, impaired glucose tolerance [IGT], then referred to as borderline diabetes, was recognized as a risk factor for both $\mathrm{T} 2 \mathrm{D}$ and cardiovascular disease. The first studies examined the effects of glucose-lowering pharmacological agents and diet restriction in men with IGT on T2D incidence (Jarrett, Keen, Fuller, \& McCartney, 1979; Sartor et al., 1980). Although methodological limitations were noted, both found 
associations between reduced dietary intake and T2D incidence. Higher baseline glucose values were also predictive of progression to diabetes (Jarrett et al., 1979). In contrast to pharmacological and dietary interventions, Long et al. (1994) conducted a quasi-experimental study comparing the effects of bariatric surgery in morbidly obese individuals with IGT to that of usual care on T2D progression. T2D was significantly reduced in the surgical group. This study contributed to a finding that remains well received today: weight loss reduces the onset of T2D.

The pharmacological and surgical interventions described were not without risks and were conducted with small, homogeneous samples. Internationally, several randomized control trials testing behavioral interventions made significant contributions to the diabetes prevention literature (Eriksson et al., 1999; Knowler et al., 2002; Pan et al., 1997; Tuomilehto et al., 2001). Pan et al. (1997) examined the effectiveness of dietary and exercise interventions in reducing the incidence of T2D in subjects with IGT in Da Qing, China. Adult men and women were randomized into one of four arms: exercise, diet, diet plus exercise or a control group (Pan et al., 1997). Thirty-three health clinics participated in the study, with each clinic randomized to intervention type. Subjects in intervention arms received individual nutritional and/or exercise counseling at the onset of the intervention; small group counseling was then conducted in decreasing intensity from weekly, to monthly and, finally, every three months for the remainder of the study. Counseling was provided by trained physicians, nurses and technicians. The control group received written brochures on diet and exercise and a one-time individual educational encounter. At six years, a 33\% reduction in T2D incidence was found in the diet only group, $47 \%$ in the exercise only 
group and 38\% in the diet plus exercise intervention group (Pan et al., 1997).

Similar to the Da Qing trial, the Finnish Diabetes Prevention Study [DPS], tested the effectiveness of lifestyle counseling in reducing the incidence of T2D (Tuomilehto et al., 2001). Subjects were randomly assigned to receive individual lifestyle counseling or to the control group that received verbal and written dietary and exercise advice. Unlike the Da Qing diet and exercise interventions, the DPS lifestyle counseling intervention had specific goals for reducing weight by $5 \%$, decreasing fat intake to less than $30 \%$ of total calories and performing 30 minutes of moderate intensity exercise per day. These goals were established based on a feasibility study which found significant improvement in glucose tolerance in subjects who lost weight and increased functional oxygen capacity through fitness training (Eriksson et al., 1999). The lifestyle counseling intervention was individually tailored and was administered by nutritionists individually weekly for seven weeks and then at three month intervals for the first year at which time the effectiveness of the intervention was assessed (Lindström et al., 2003). At completion, the cumulative incidence of T2D was $58 \%$ lower in the group randomized to lifestyle counseling intervention.

The landmark Diabetes Prevention Program [DPP] (2002) clinical trial has had the most impact on diabetes prevention in the U.S. This study addressed several previous limitations, particularly issues related to external validity. Previously, diabetes risk was defined clinically by the presence of IGT. This is consistent with World Health Organization [WHO] (1985) criteria of a plasma glucose level between 140-200 mg/dl following a $75 \mathrm{~g}$ oral glucose tolerance test. Evidence has since revealed that reliance on IGT alone to clinically predict future T2D misses a significant subset of the 
population at risk. The presence of an elevated or impaired fasting glucose [IFG] was found to have a higher 5-year T2D incidence rate than that of IGT (Gabir et al., 2000). In 2000, the American Diabetes Association [ADA] established additional criteria for elevated risk of T2D as the existence of a fasting plasma glucose in the range of 95$125 \mathrm{mg} / \mathrm{dl}$ (Gavin et al., 1997). Inclusion criteria for the DPP reflected these new guidelines. Previous study populations were homogenous. The DPP recruited a representative sample to include diverse racial and ethnic groups, both younger and older individuals and more diversity in socioeconomic position. Lastly, the DPP included a pharmacologic arm using a class of glucose lowering agents not previously compared to ILI.

The DPPRG randomized 3,234 subjects in 27 participating research sites nationally. DPP ILI goals were twofold: 5-7\% loss of total body weight and performance of 150 minutes of physical activity per week. Subjects were randomized to one of three study arms. One arm received the biguanide metformin $(n=1073)$ with standard lifestyle advice. The remaining two study arms were ILI and a control group which received standard lifestyle advice with placebo. Diabetes incidence differed significantly among the three groups with a $58 \%$ reduction in T2D incidence, with a mean weight loss of 5.6kg in the ILI arm and a 31\% reduction in the metformin arm (Knowler et al., 2002). Seventy-four percent of subjects in the ILI arm met the physical activity goal.

The DPPRG utilized a curriculum entitled Lifestyle Balance developed by the DPP Lifestyle Balance Core at the University of Pittsburgh Medical Center (DPP Research Group, 2002). It consisted of 16 core sessions taught by Master's-prepared dieticians, 
exercise physiologists, physicians, nurses and behavioral psychologists. Sessions were completed over the course of 20-24 weeks. In addition to the Lifestyle Balance curriculum, patients in the intervention group were assigned to a lifestyle coach who gave them individual counseling and feedback. Case managers of the same ethnic background were assigned to ethnically diverse subjects. Participants worked toward achieving predetermined goals of a $7 \%$ weight loss and performance of 150 minutes of physical activity per week with the aid of personal trainers (DPP Research Group, 2002). Supervised physical activity sessions were offered twice weekly. Following the core sessions, facilitators met with individuals bi-monthly and maintained weekly telephone contact. Monthly group meetings were held up to one year. A toolbox was available for each member in the lifestyle group that contained exercise videos, grocery coupons or cookbooks. Toolboxes cost $\$ 100.00$ per subject. Over the threeyear research period, total cost per subject in the lifestyle group was $\$ 2,780.00$ (DPP Research Group, 2003).

It is important to note that pharmacological intervention in pre-diabetic individuals continues to be less effective than ILI. Decreased insulin sensitivity and inadequate insulin secretion are hallmarks of pre-diabetes and T2D. The former refers to the uptake of glucose by the cell which is mediated by insulin; the latter is characterized by pancreatic $\beta$-cell dysfunction, such as a sluggish first phase insulin response to glucose. Compared to metformin alone, ILI was significantly more effective in improving both of these pathologies in the DPP and subsequent studies (Kitabchi et al., 2005; Knowler et al., 2002a; Knowler et al., 2002b). Studies equally successful in reducing the incidence of T2D with medications did so only when used in combination 
with lifestyle intervention (Ramachandran et al., 2006; Torgerson, Hauptman, Boldrin, $\&$ Sjostrom, 2004). Metformin, the most commonly used drug to treat pre-diabetes, has documented side effects that are not well tolerated. While new and improved pharmaceutical options are likely to become available, access and cost will be limiting factors. Glucose lowering medications do not cure insulin resistance or restore $\beta$-cell loss. Discontinuation in the absence of lifestyle changes results in resurgence of elevated blood glucose levels.

The above studies had a significant impact on diabetes prevention research and how it is approached today. Diabetes incidence was significantly reduced with a 5$10 \%$ loss of total body weight establishing a physiological threshold necessary to halt the progression of T2D. Physical activity of at least 150 minutes per week was a significant predictor of weight loss. In isolation, health education and nutritional counseling were not effective. Knowledge alone does not necessarily lead to health behavior change; a combined approach of education and behavioral support was necessary. Pharmaceutical intervention alone was not as effective as ILI. Lastly, intensity mattered. Frequent contact with subjects, individualization and a maintenance period were beneficial. Together, these studies support the primary argument for ILI for diabetes prevention; they were safe and effective (Ali, EchouffoTcheugui, \& Williamson, 2012; Knowler et al., 2002a; Norris et al., 2005; Yamaoka \& Tango, 2005; Yoon et al., 2013).

\section{Translational ILI in Community Settings}

The primary aim of the randomized control trials discussed above was to test the hypothesis that T2D could be prevented or delayed in pre-diabetic subjects with ILI. 
The DPP, in particular, was an efficacy trial that was not intended to be translated into community settings or any other type of program (DPP Research Group, 2002; Pronk, Boucher, Jeffery, Sherwood, \& Boyle, 2004). The significance of the findings however, could not ethically be ignored. Efficacy trials by definition, occur in controlled conditions, whereas effectiveness trials are a measure of whether an intervention remains safe and effective "under real world conditions" (Glasgow, Lichtenstein \& Marcus, 2003, p.1261). Translation efforts became a research priority to determine effectiveness across practice settings. The literature reveals a range of modifications or adaptations to the evidence-based ILI in community settings where the primary outcome for most has shifted to weight loss with the rationale that each kilogram of weigh lost reduces T2D incidence by 16\% (Hamman et al., 2006).

Ackermann et al. (2008) conducted the Diabetes Education and Prevention with Lifestyle Intervention Offered at the YMCA [DEPLOY] study (Ackermann, Finch, Brizendine, Zhou, \& Marrero, 2008. This was an academic and community partnership between the Indian University School of Medicine and the YMCA. Ninety-two pre-diabetic adults were cluster-randomized into ILI or a control group. This study closely emulated the evidence-based ILI with the exception of using a group format. Findings included a $6 \%$ weight loss in the intervention group compared to a $2 \%$ weight loss in the control at 6 months. At the 12 -month follow up, there were declines in percent weight loss, but weight loss remained significant.

The Healthy Living Partnerships to Prevent Diabetes [HELP PD] was a randomized trial that aimed to expand an existing diabetes education program into community settings (Katula et al., 2010). This study was more intensive in terms of 
dose and duration of program; 24 core sessions were followed by an 18-month maintenance component with two monthly individual contacts in the intervention group. Community health workers [CHWs] delivered the intervention with the assistance of a registered dietician. These CHWs were community members with wellcontrolled T2D and had adopted healthy eating and physical activity behaviors. CHWs were guided by a DVD developed by the research team and adapted from the DPP curriculum. Despite the lengthy intervention, attrition rates were low. Attendance at core sessions was also low. The intervention group had a mean weight loss of $7.3 \%$ at 12 months compared to $2 \%$ in the control. In contrast to other translational interventions, HELP PD reported significant reductions in fasting blood glucose at 12 and 24 months. Weight loss was positively correlated to these reductions. The sample was adequately powered and was comprised of predominately educated white females (Katula et al., 2011; Katula et al., 2013). HELP PD provides evidence that ILI can be effective in community settings with its major limitation being that it remains resource intensive. The use of CHW as facilitators was reported as a cost reduction measure.

In Montana, the Diabetes Control Program of the Montana Department of Health funded a feasibility study conducted at four health care agencies to include one community partnership with the YMCA (Amundson et al., 2009). A single group prospective design was used to evaluate the feasibility of a translational ILI with adults at high risk for T2D. The evidence-based model was adapted for use in a group format. The mean weight loss was $6.7 \%$ of total body weight; $70 \%$ of participants met physical activity goals. The Montana Department of Health also partnered with a rural medically underserved community using a similar approach. Weight loss at 4 months 
was greater than $7 \%$ in $52 \%$ of participants and remained the same at the 12 -month follow up. This study, utilizing a one group time series design, included two supervised physical activity sessions a week with multiple choices available to include dancing, water aerobics and kick boxing through local community partnerships (Vadheim et al., 2010). This was associated with a large percentage of the sample meeting physical activity goals.

Members of the original DPPRG developed a comprehensive translational model proposed as a means to standardize translational ILI in community settings (Kramer et al., 2009). This model, Group Lifestyle Balance [GLB], was referred to as an adaptation of the DPP ILI. Changes were made to make the curriculum more consistent with current dietary recommendations and also to reduce the number of core sessions from 16 to 12 (Kramer et al., 2009). Despite adaptations, GLB maintains what has been identified by Venditti \& Kramer (2012) as necessary components of evidence-based ILI. A necessary component was defined as "one, that by and large, must be present for the desired outcome to follow" (Venditti \& Kramer, 2012, p.139). These include goals for weight loss and physical activity consistent with DPP findings, a curriculum that integrates nutrition, physical activity and behavioral strategies, facilitators who are trained health care professionals, strong emphasis on self monitoring and problem solving skills and a minimum dose and duration (Kramer et al., 2009).

Seidel, Powell, Zgibor, Siminerio and Piatt (2008) applied the GLB model with an urban medically underserved community. Using a non-randomized single group design, 88 adult men and women received an adapted intervention with 12 core 
sessions offered over 12-14 weeks. This study did not utilize a maintenance phase following core sessions. The intervention was facilitated by a dietician and exercise physiologist with the assistance of lay health coaches. The role of coaches was to provide clarification and enhance communication (Seidel et al., 2008). One third of participants achieved a 7\% weight loss at 6 months; one half lost 5\%. At the two-year follow up, over half of those that had a $5 \%$ weight loss were able to maintain that weight loss (Piatt, Seidel, Chen, Powell, \& Zgibor, 2012). Participants reported having significant difficulty completing dietary and physical activity logs, which resulted in the inability to analyze these data. The GLB model has been used to test the effectiveness of program delivery by certified diabetes educators with similar outcomes (Kramer, McWilliams, Chen, \& Siminerio, 2011), and in comparative effectiveness research to test the effectiveness of alternative ILI formats (Piatt, Seidel, Powell, \& Zgibor, 2013). In the latter, ILI was compared to receiving ILI through a DVD or via DVD plus internet materials. Subjects who were able to self-select into a preferred ILI format sustained greater weight loss. Subjects who received the inperson ILI, however, had greater amounts of sustained weight loss at six months.

The GLB model was also used to test the feasibility and effectiveness of an ILI for Latina women in an urban community setting (O’Brien et al. 2015). A single arm design was used with a sample $(\mathrm{n}=20)$ described as socioeconomically challenged middle-aged Latina women. The GLB model was modified to be facilitated in Spanish by community health workers or promotoras. Promotoras received 18 hours of training and held no more than a high school diploma (O'Brien et al., 2015). This study, entitled the Promotora Led-DPP (PL-DPP), consisted of 24 sessions and had a primary 
outcome of a $7 \%$ weight loss. Mean weight loss at one year was $5.6 \%$ which was clinically and statistically significant. Authors attributed the success of this pilot study to its fidelity to the GLB model. One aspect that sets this study apart from others was that it assessed health literacy pre and post intervention and reported marginally significant differences $(p=0.05)$. Major limitations of this study include its single arm design and extremely small sample size. In addition, physical activity goals and outcomes were not reported in this study.

Translational studies were conducted in community faith-based settings (Boltri et al., 2008; Boltri, Davis-Smith, Okosun, Seale, \& Foster, 2011; Davis-Smith et al., 2007). Using a community-based participatory approach, researchers at Mercer University School of Medicine and a rural African-American church in Georgia conducted several studies. Two feasibility studies were conducted using one group pre-post designs. One tested a 6-session intervention, the second a 16-session intervention. Sample sizes were extremely small, with 10 participants in the former and 8 in the latter. Both adapted materials from the DPP Lifestyle Balance curriculum while tailoring the intervention based on formative research (Boltri et al., 2006). The modified six-session curriculum was not described. Core sessions included opening and closing prayers, weekly goal setting for physical activity and diet, and peer support. Interventions were facilitated by a volunteer health professional not otherwise identified. Both interventions experienced weight loss, though not clinically meaningful, at 6 and 12 months. Physical activity was not reported. Using a nonequivalent comparison group design $(n=37)$, the same investigators compared the two interventions in relation to clinical outcomes and cost effectiveness (Boltri et al., 
2011). Both groups experienced weight loss and improved fasting glucose with insignificant between group differences. The cost of materials was cited as $\$ 1,075$ for the more intensive intervention compared to $\$ 934$ for the brief intervention.

Ruggiero, Oros and Choi (2011) conducted a non-randomized prospective study in an underserved Hispanic community in Chicago, IL. The sample included 69 adults, of which $92.8 \%$ were women. Inclusion criteria for the study was an elevated body mass index and Hispanic ethnicity with the rationale that the two factors are known risk factors for T2D. The intervention entitled the Healthy Living Program [HLP] was developed through an academic-community partnership using a community-based participatory approach. HLP was reported as an adaptation of the DPP ILI that was tailored to "provide culturally specific information on diabetes risk" (Ruggiero et al., 2011, p.567). The DPP Lifestyle Balance curriculum was used in Spanish and supplemental educational materials were adapted to make them culturally relevant. Sixteen weekly core sessions were provided with six monthly sessions thereafter. Sixmonth outcomes included a $7 \%$ weight loss in $20 \%$ of participants. At 12 months, $16 \%$ of participants maintained this weight loss. There were no significant changes in physical activity behaviors. The most significant modification was the use of a bilingual Healthy Lifestyle Coach with extensive training in diabetes education to facilitate the program (Ruggiero et al., 2011).

Mau et al. (2010) conducted a study with a Pacific Islander population using a single group pre-, post-design. Using a CBPR approach, the ILI was designed, implemented and evaluated by community members and faculty at the University of Hawaii. Modifications were made to the DPP ILI and were summarized as (1) use of 
the group format, (2) CHWs as facilitators, (3) reduction in dose and duration, (4) addition of topics provided by formative research findings and, (5) use of simple language (Mau et al., 2010). Results at 12 weeks included a modest mean weight loss of 1.5 kilograms. Behavioral outcomes for dietary fat and physical activity were reported as improved from baseline. Notable in this study was a positive association between number of core sessions attended and amount of weight loss. This was limited, however, by the duration of the study.

The translational studies reviewed thus far are instrumental in that they establish the feasibility of evidence-based ILI in community settings. They also provide support for implementing evidence-based ILI in the group format. Yet, most studies lacked a comparison group with single group pre- post-designs predominating. Evidence of sustainability was also limited. Pilot or feasibility studies made up the majority; sample sizes were small and underpowered to conduct subgroup analyses. Study samples were comprised of predominantly non-Hispanic white women with some evidence of access to health care. Although not turned away based on ability to pay, programs incurred a cost to participants (Ackermann et al., 2008; Amundson et al., 2009; Vadheim et al., 2010). These studies proposed reimbursement from public and private health insurers as a solution for sustainability and were undoubtedly driving forces in the creation of the National Diabetes Prevention Program [NDPP].

More rigorous research methods that result in sustainable options come at a significant cost and are not always feasible or ethical in research (Kramer et al., 2011). The limitations of the studies reviewed thus far speak to the potential difficulties in translation, particularly in low resource community settings. They were costly to 
implement despite large cost reductions from the DPP ILI which was estimated at $\$ 1,400.00$ per subject annually (Hernan et al., 2003). Costs per participant were rarely reported; when reported, they ranged from $\$ 300-430$ per participant annually (Kramer et al., 2009; Kramer et al., 2011; Lawlor et al., 2013). The evidence-based model was resource intensive and costly. ILI, however, remained cost effective as compared to the lifetime costs incurred in treating T2D and its complications ( $\mathrm{Li}$, Zhang, Barker, Chowdhury, \& Zhang, 2010). In question is whether strict adherence to the evidencebased model is necessary to achieve similar outcomes with populations at highest risk for T2D, such as the low income Hispanic population.

At the time of this review, three studies were identified using more rigorous research designs and highly adaptive approaches to ILI with high-risk Hispanic populations at high risk for T2D in community settings. Kanaya et al. (2012) tested the effect of a less intensive telephone-based approach augmented by group workshops on the reduction of T2D risk factors in a low income Hispanic community. The intervention, Live Well Be Well [LWBL], was academic-public health department collaboration in California. Adults with pre-diabetes were randomized to LWBL or a delayed intervention. Structural components included twelve phone calls, two face-toface contacts with a trained counselor and five voluntary group workshops. Total contact time was 12 months. This program was unique in that it was "choice-based and individually tailored" (Delgadillo et al., 2010, p.642). Materials and counseling subjects received were based on individual preferences and stage of change (Delgadillo et al., 2010). Materials were adapted from existing resources and altered to accommodate lower literacy levels. Emphasis was on self-monitoring, goal setting and 
problem solving. The authors identified education, skills building and motivational interviewing as key program components (Kanaya et al., 2012). Weight loss was statistically significant between treatment groups but modest in relation to clinical recommendations. There were no differences in physical activity between groups.

The Lawrence Latino DPP (2012) was an academic-community partnership in Massachusetts. High-risk Latino adults were randomized to ILI or a delayed intervention. The intervention included 13 core sessions using literacy and culturally appropriate materials presented in a group format and 3 home visits (Merriam et al., 2009). The intervention was described as culturally tailored and culturally adapted. This included integrating traditional food preferences and exploring cultural beliefs and attitudes towards diabetes prevention. Content was visually oriented and simple. Foods were color-coded to illustrate fat and carbohydrate content. A novella, or soap opera, was made in Spanish depicting cultural attitudes towards preventing T2D. Key components were education and skill development, specifically goal setting, selfmonitoring and problem solving (Merriam et al., 2009). Program facilitators were Spanish-speaking individuals who had undergraduate training in nutrition. Findings after 12 months included modest weight loss with significant improvement in hemoglobin A1C. Physical activity goals were not met in this study.

Project Help Educate to Eliminate Diabetes [HEED] was conducted in a low income predominantly Black and Latino community in Harlem, NY (Horowitz, Eckhardt, Talavera, Goytia, \& Lorig, 2011). This was an academic-community partnership that used a CBPR approach in this pilot study. A community board participated from the selection of the health disparity to be addressed through 
planning, intervention development, implementation and evaluation. A major design goal was to make the program economical and therefore sustainable (Horowitz et al., 2011). An existing chronic disease management curriculum was adapted to make it culturally sensitive and suitable for low literacy levels. High-risk adults were randomized to the intervention or a delayed intervention. Focus was on the creation of simple action plans with short- and long-term goals. The intervention was facilitated by peer leaders in the group format for eight core sessions. Dancing was offered as a "culturally appropriate" form of physical activity (Horowitz et al., 2011, p.448). The mean weight loss was $4.3 \%$ in the intervention group as compared to $1.5 \%$ in the control group. No between-group differences were reported in physical activity.

\section{Synthesis of Translational Research}

Translation of the evidence-based ILI in community settings was evident in the literature. The literature review revealed that studies consistently adhered to several core components: (1) weight loss was targeted through physical activity and diet, (2) a designated curriculum was presented over time, (3) in-person support was provided from a facilitator and, (4) behavioral strategies were emphasized to facilitate change. There was significant variation, however, within these elements. Components such as an individualized approach and dose and duration varied the most.

Interventions always included an educational component provided over a minimum of six weeks. The means in which material was presented was not always clear though studies consistently reported using a curriculum with instructional materials. One study described sessions as "participatory and interactive" (Amundson et al., 2009, p.213), while the term workshop was used in another, implying an 
informal approach to presenting material (Kanaya et al., 2012). Studies with low income Hispanic populations reported using materials that were appropriate for low levels of literacy with development and testing of materials prior to the intervention (Ockene et al., 2012). Interestingly, knowledge was not a variable reported in the studies reviewed. Studies uniformly reported nutritional education on recommended fruit and vegetable consumption, identifying fats in the diet and basic principles of physical activity. Those providing a variety of structured physical activity experiences reported better outcomes for that variable (Amundson et al., 2009; Vadheim et al., 2010). This was in contrast to studies that provided verbal physical activity instruction only which did not fare as well (Ockene et al., 2012; Seidel et al., 2008). Physical activity outcomes were consistently not met in studies conducted with low income Hispanic populations. This is extremely relevant in terms of diabetes prevention due to its powerful effects on insulin sensitivity and glucose tolerance and also the positive association between physical activity and the reduction in cardiovascular (CVD) risk factors (e.g. hyperlipidemina, hypertension) that co-exist with pre-diabetes and metabolic syndrome.

Translational studies conducted in community settings were predominantly conducted with groups of participants. The rationale for the group format was noted as a cost saving measure and was also noted as a means for members of the group to “provide motivational support" to one another (Davis-Smith et al., 2007, p.443). In describing their methodology with a low-income Caribbean Latino population, Merriam et al. (2009) reported that group members offered solutions to one another. The DPPRG cited that individual facilitator to participant contact was critical to the 
success of the DPP. This is unsustainable and unrealistic, particularly in community settings. Several studies integrated the group format with individual encounters, home visits or telephone counseling sessions (Kanaya et al., 2012; Katula et al., 2011b; Ockene et al., 2012). This combined approach was more effective in behavioral obesity interventions for multiethnic, minority adults (Seo \& Sa, 2008), and may have been an attempt to provide this individual approach and improve outcomes. Of those studies, the highly intensive ILI (in terms of number of group and individual contacts) conducted by Katula et al. (2011) reported significant weight loss at 12 months. Home visits were used in a low-income Hispanic population to enhance attendance (Ockene et al., 2012); this was not examined for its impact on processes or outcomes. The actual impact of the group format or group support on participant outcomes was not examined in any of the studies.

The literature reflects disagreement in regards to dose and duration for ILI to prevent T2D. The dose referred to the number of core sessions provided intensively at first and then tapered off. In a systematic review conducted by Ali, EchouffoTcheugui and Williamson (2012), ILI with more core sessions had higher attendance rates with an increase in weight loss with each additional session attended. The same review found that ILI with a minimum of 16 sessions was more effective and is consistent with previous reviews (Norris et al., 2005; Satterfield et al., 2003). Venditti and Kramer (2012) posited that ILI should consist of a minimum of 16 core sessions and also support Katula et al.'s (2010) model of 24 core sessions. In this review, attendance was positively associated with weight loss in at least two studies (Mau et al., 2010; Ockene et al., 2012). Duration was defined as the total time of contact with 
participants. In the evidence-based ILI, a maintenance period followed core sessions for up to one year. In this review, a maintenance period was the most common aspect or component to be eliminated and reflecting the need to reduce overall cost. ILI with a total duration of at least nine months was the most effective providing rationale for extending program duration when feasible (Ali et al., 2013). One randomized control trial conducted with a low-income minority population provided a maintenance phase, yet lacked significant differences in weight at 12 months (Kanaya et al., 2012). Other studies conducted with low income Hispanic populations had fewer core sessions and did not include a maintenance phase (Ockene et al., 2012; Parikh et al., 2010). While there were significant differences between groups, mean weight loss was less than evidence-based recommendations.

ILIs for the prevention of T2D have been described as behavioral interventions (Norris et al., 2005; Venditti \& Kramer, 2012; Whittemore, 2011), the defining features of which included the combined use of education, social support and cognitive behavioral strategies (Norris et al., 2005). Social support was operationalized as frequent contact with a program facilitator or counselor. The concept of social support was also referred to as a behavioral strategy, e.g., individuals should seek out sources of support (Delgadillo et al., 2010; Venditti \& Kramer, 2012). The most effective studies (clinically meaningful and significant weight loss at 12 months) utilized an ILI design with 12 or more in-person core contacts and a minimum of monthly contact for an additional 3-6 months (Ackermann et al., 2008; Katula et al., 2011). In contrast, programs that utilized electronic core sessions such as email or a DVD format or relied on telephone counseling were not as effective 
sessions conducted in person (Kanaya et al., 2012; Kramer et al., 2010). Postintervention follow up contact from a facilitator or $\mathrm{CHW}$ via telephone or email had results similar to in-person follow up. ILIs conducted with low-income Hispanic populations were more likely to be facilitated by $\mathrm{CHWs}$. In their systematic review Ali et al. (2012) found that ILIs facilitated by CHWs were as effective as those conducted by healthcare professionals. This holds promise for future ILI in low-resource community settings. The concept of social support was not otherwise reported or analyzed in relation to clinical or behavioral outcomes in the studies reviewed.

Behavioral strategies most frequently reported in community translations were goal setting and self-monitoring. Subjects were encouraged to set weight loss and physical activity goals and to self-monitor fat and fruit and vegetable consumption. Interestingly, it was not always clear if the goals were those of participants or merely reflected the goals empirically established by randomized trials such as the DPP. The LWBW study conducted with a low income Hispanic population reported allowing participants to choose one achievable goal with the rationale that self-selected goals would align with readiness leading to increased confidence (Delgadillo et al., 2010). Here, researchers reported a willingness to sacrifice study efficacy in order to help participants achieve realistic and attainable goals. Self-monitoring by keeping a log or journal has been found to be the most effective behavioral strategy for modifying dietary intake and maintaining weight loss outside of the diabetes prevention literature (Burke, Wang, \& Sevick, 2011; Butryn, Phelan, Hill, \& Wing, 2007). Participants in the reviewed studies were encouraged to self-monitor dietary fat intake and physical activity in some but not all studies. Log books, handouts and pedometers were 
frequently provided for this, yet few studies actually reported the extent to which subjects self-monitored. Seidel et al. (2008) noted that participants in a low income community had significant difficulty completing self-monitoring logs, whereas the DEPLOY (2008) study conducted in partnership with the YMCA found that men and participants over 60 were more likely to keep dietary logs. Participants who completed dietary logs for fat were eight times as likely to achieve weight loss goals (Amundson et al., 2009). Self-monitoring in this study was also associated with achieving physical activity goals.

Additional cognitive behavioral strategies such as problem solving, skills building and creating action plans were cited. In an ILI (Project HEED) adapted for an underserved Latino population in Harlem, NY, Parikh et al. (2010) reported using action plans with small achievable goals as a strategy to increase self-efficacy. Participants reported no changes in physical activity or fat intake, however, at study completion. LWBL also used short term action plans developed by participants with facilitators. The impact of this strategy was not reported.

In this review, there was significant heterogeneity in translation of the evidencebased model in general. There were notable trends, however, in the few studies conducted with high-risk Hispanic populations. ILIs were more likely to be of shorter dose and duration with a range of core sessions from 8 through 16. All ILIs were facilitated at least in part by CHWs. Social support in the form of individual counseling was often provided in addition to group sessions; however, it was not clear if this was beneficial. The most frequently cited behavioral strategies were selfmonitoring and goal setting. Self-monitoring in particular was difficult for 
participants. Behavioral variables were not consistently measured; studies that measured behavioral variables did not report positive findings in relation to outcomes. In terms of clinical outcomes, weight loss was significant between groups in randomized studies. Weight loss, however, was not consistent with the amount needed to prevent T2D when measured at 12 months. The evidence was not conclusive for weight loss maintenance due to shorter study durations. Physical activity as a behavioral target was unrealized, and there was significant variability in the adoption of dietary changes. Despite poor session attendance, study retention was favorable. For example, Ockene et al. (2012) reported a 94\% retention rate and Kanaya et al. (2012) 93\% retention at the 12-month follow up.

As evidenced above, it was difficult to infer whether adapting the evidence-based ILI has proven beneficial and which components had the most impact with high-risk Hispanic populations. An argument has been made regarding fidelity to the evidencebased model (Venditti \& Kramer, 2012). Much of the diabetes prevention literature supports a standardized approach to ILI consistent with the DPP, which was representative of the U.S. population and equally efficacious among subgroups. A counterargument is that issues related to a particular intervention —in the case of ILI weight, physical activity and diet—are culturally and contextually influenced and therefore cannot be addressed with a "one size fits all" approach (Kumanyika, 2008, p.583). These views reflect the range of theoretical perspectives that have shaped ILI for the prevention of T2D historically and in translation.

\section{Theoretical Influences on ILI}

Behavioral perspective. Diabetes prevention through lifestyle intervention has its 
theoretical roots within the discipline of psychology and has predominantly been viewed from this behavioral perspective. Although the intent has been to decrease the incidence and prevalence of T2D, the focus has been on changing behavior in order to achieve this. Broader theoretical perspectives have been integrated particularly in translational ILI in communities most affected by health disparities, yet the behaviorist paradigm exerts considerable influence on ILI regardless of population.

To include the DPP, the early diabetes prevention trials utilized strategies consistent with several psychological theories or models, often without explicitly citing any one theory. Principles of behavior modification were frequently cited, which can be traced to both the stimulus-response theories of behaviorism and cognitive behaviorism (Skinner, 1963; Watson, 1913). Behavior modification refers to specific techniques or strategies employed by an individual to alter a faulty behavior (Schwartz, 1982). Classic behaviorism assumes that (1) behaviors are observable and quantifiable entities devoid of conscious thought on the part of the individual; (2) behavioral antecedents or stimuli and their consequences regulate behavior; and (3) behavior is capable of manipulation and control by altering environmental stimuli, such as removing negative or adding positive triggers that lead to certain behaviors (Skinner, 1963; Watson, 1913). Faulty behaviors, or operants, are extinguished by using negative reinforcers, such as taking away environmental cues, and positive reinforcers, such as adding cues to perform a desired behavior.

The behaviorist perspective is deterministic; behavior as an event is preceded by antecedents that will consistently produce stable outcomes. The concept of free will as it relates to learned behavior is absent. Humans are capable of cognition, emotion and 
beliefs, yet these are unscientific and have no effect on overt behavior (Skinner, 1977). Cognitive psychologists reject the behaviorist notion that individuals operate purely from a conditioned state over which they have no control; they are not "weathervanes" automatically shaped and controlled by external stimuli (Bandura, 1986; Bandura, 1991). Humans adapt or change their behavior after mentally processing information they receive from the social world together with conceptions about the self. Unseen cognitive processes such as beliefs, attitudes and emotions can potentially directly affect or mediate behavior (Bandura, 1986; Beck, 1964; D'Zurilla \& Goldfried, 1971; Ellis, 1980). From this humanistic perspective, humans are conceptualized as thinkers who are in control of their own behavior; they possess human agency. A cognitive behavioral perspective provided novel theoretical explanations for objective behavior which in turn expanded the means in which to intervene. With this came the proliferation of theories or models now referred to as behavior change theories that attempt to predict and explain behavior and also propose to guide the process of change.

Social Cognitive Theory $[\mathrm{SCT}]$ is the most cited theoretical influence in the translational ILI literature and, though not explicitly cited in the evidence-based model, is conceptually consistent. According to Bandura (2006), "to be an agent is to influence intentionally one's functioning and life circumstances" (p.270). Environments do not passively exert their influence on human behavior; rather, a social cognitive or interactionist perspective proposes a triadic relationship between individual, environment and the behavior (Bandura, 1986). Gone is the duality between environment and behavior. Behavior is socially mediated and culturally 
contextual, yet remains thoughtful, purposeful. There are four properties of human agency: intentionality, forethought, self-reactiveness and self-reflectiveness (Bandura, 1991; Bandura, 2004). The properties of human agency are cognitive processes that reflect individuals' ability to envision themselves in the future, make judgments about ability, predict outcomes based on a behavior, make goals and actualize those goals through planning and strategizing (Bandura, 2006). Individuals have control over behavior, but are also in control of how they think and feel about a behavior which ultimately affects performance. Two central constructs of SCT integral to behavior change are self-efficacy and self-regulation.

Bandura (2004) referred to self-efficacy as "the foundation of human motivation and action"(p.144). Self-efficacy is one's confidence or belief in the ability to perform a behavior. Belief in personal ability can serve as motivator or deterrent. Self-efficacy also predicts effort and the ability to persevere. Self-efficacy is context dependent meaning one may have high self-efficacy in achieving weight loss, but low selfefficacy in being physically active. Self-efficacy mediates behavior, and also exerts its influence by indirect pathways. When proximal goals are achieved, self-efficacy is enhanced favoring distal goal attainment. Breaking down larger goals into smaller, more achievable goals is associated with a sense of mastery which leads to greater self-efficacy (Michie, Abraham, Whittington, \& McAteer, 2009). This strategy is particularly important in diabetes prevention, where modest weight loss has a significant impact on delaying or preventing diabetes. The ability to overcome perceived barriers and to mobilize facilitators to change further serves to increase selfefficacy. 
SCT proposes that belief and intention alone are inadequate for behavior change. Purposeful action is necessary to influence change (Bandura, 1991). Self-regulation is an action-oriented construct that directs one's attention to the desired behavior. Selfregulatory mechanisms are a system of sub-functions that include self-monitoring, goal setting and problem solving. Self-monitoring refers to increasing awareness of environmental cues and to emotional and social cues related to a behavior. When individuals self-monitor, they recognize patterns of behavior which they can begin to alter. To self-monitor is to also identify beliefs or perceptions and recognize how they impact behavior. Self-monitoring also affects goal setting and problem solving. In their review of data collected from the National Weight Control Registry, Wing and Phelan (2005) identified self-monitoring as a key strategy used by individuals for both weight loss and weight loss maintenance for greater than one year.

Bandura (2004) described a core set of determinants for health behavior change to include (1) knowledge of health risks and the benefits of health related practice, (2) one's belief in the ability to exert control over health, (3) positive outcome expectations regarding benefits and costs, (4) goals with plans and strategies for reaching them and (5) one's perception of the facilitators and real or potential barriers to making health related changes. These determinants provide a framework for formative intervention development. In a low income predominantly Caribbean Latino community, Rosal, Borg, Bodenlos, Tellez and Ockene (2011) conducted focus groups to explore risk awareness and severity of T2D, perceived risk factors, knowledge regarding the prevention of $\mathrm{T} 2 \mathrm{D}$ and barriers and facilitators to prevention practices. This predominantly female purposive sample was knowledgeable about T2D and its 
complications. Participants, however, did not believe themselves to be at risk for T2D. Family history was attributed to risk, whereas ethnicity and race were not. Risk was also perceived to be related to stress and the hurried lifestyle associated with living in the U.S. Of particular importance, poor nutrition was perceived to increase the risk of T2D. Participants noted that physical activity aided in the prevention of T2D but not weight loss. Participants rarely used nutritional practices and physical activity in combination in efforts to lose weight. Barriers to weight loss were related economics, time, stress and work schedules (Rosal et al., 2011)

The concepts of stimulus control and reinforcement are not abandoned in SCT, yet they are defined in terms of the cognitive self. Increased awareness of emotional stimuli aids in directing attention to the behavior. Rewards are effective when they are self-determined and intrinsic rather than influenced by an external source. When external support is provided in an endeavor, it is ultimately the individual who determines human action in a self-directed manner (Bandura, 1986).

SCT is not without limitations. There are multiple constructs of which only a select few have been empirically validated (Baranowski, Perry, \& Parcel, 2002). Selfregulation (through self-monitoring and goal setting) and self-efficacy were frequently cited behavioral strategies in translational ILI. Making small achievable goals as means to increase self-efficacy was identified as a useful strategy with low income populations (Michie, Jochelson, Markham, \& Bridle, 2009), and in increasing physical activity in Hispanic women (Larsen et al., 2014), but was not found in this literature review when assessed as a secondary outcome measure (Parikh et al., 2010).

It has been proposed that SCT is a universal theory. It reflects attributes that are 
innately human; therefore, it is cross-cultural (Resnicow, Braithwaite, Dilorio, \& Glanz, 2002). Bandura (2004) rejected the polarity of individualism versus collectivism and instead argued that although culture is influential to one's sense of efficacy, it is the concept of agency, or personal control, which ultimately mediates efficacy expectations. An argument can also be made that the perception of having control over oneself or situation is not universal. Social position and social context have the potential to influence one's perception of control over outcomes. An expanded definition of agency to include personal, proxy and collective agency attempts to resolve this. Personal agency is the ability to make connections between individual action and its consequences and to differentiate one's actions from those of others (Bandura, 2006). Personal agents determine their own actions and have control over "thoughts, feelings, motivations and actions" (Bandura, 1991, p.249). In contrast to the unconscious nature of learning proposed by classic behaviorism, an agentic perspective views learning as a conscious process derived from direct experience and observing others. When individuals perceive themselves as powerless to social conditions, they seek outside assistance or proxy agency to act on their behalf (Bandura, 2002). Collective agency is when people work together to shape the future which can enhance efficacy beliefs. Regardless of the type of agency that is activated, change remains at the level of individual. SCT does not address altering environments on the behalf of individuals or populations. While culture is viewed as dynamic and diverse, human cognition and behavior, though socially influenced, can be explained as universal truths.

The Transtheoretical Model [TTM] and Motivational Interviewing were applied 
in the evidence-based model and were evident in translational studies in the community. The TTM blends several behavioral and psychoanalytic theories (Prochaska, Redding, \& Evers, 2002). Major assumptions are: (1) no single theory is capable of explaining behavior change; the best approach is to integrate multiple theories; (2) behavior change is a process that occurs over time in a series of stages; (3) interventions are often unsuccessful because individuals are not ready for change; and (4) specific strategies or processes should be utilized depending on the individual stage of change [SOC] (Prochaska et al., 2002). The SOC proposed by the TTM include precontemplation, contemplation, preparation, action and maintenance. Precontemplators have no intention of changing and contemplators plan to change within the next six months. Those in the preparation stage will take action in the next thirty days and those in the action stage have been performing the behavior for less than six months. Maintainers have maintained a new behavior for more than six months. The construct of the Processes of Change are the actions taken by individuals to move through the SOC (Prochaska et al., 2002). Processes include: consciousness raising, dramatic relief and environmental reevaluation (precontemplation/contemplation), self-reevaluation (contemplation/preparation), selfliberation and helping relationships, counter conditioning, reinforcement management and stimulus control or maintenance.

The DPP ILI (2002) measured stage of change for weight loss and found a positive correlation between baseline SOC and post-intervention weight loss. The TTM was applied to a limited extent in translational ILI. In this review, Ruggiero, Oros and Choi (2011) measured SOC at three time points for fruit and vegetable 
consumption, fat consumption and physical activity. Findings were significant for movement along the continuum at each time point with the exception of the 6- and 12months assessment of fruit and vegetable consumption and physical activity (Ruggiero et al., 2011). This non-randomized single group prospective study did not assess other psychological or behavioral variables. There are several limitations to application of the TTM, particularly the SOC construct, in ILI for diabetes prevention. There is little evidence that individuals in higher stages of change actually make changes any faster than those in the lower stages (Bridle et al., 2005; Resnicow, McCarty, \& Baranowski, 2003). Study duration must be sufficient enough to fully capture SOC. Participation in ILI in itself is a potential indicator of readiness. SOC is contextual which, for diabetes prevention, should include assessment of multiple behavioral variables such as weight loss, dietary behaviors and physical activity.

Motivational interviewing [MI] is a communication method to facilitate behavior change influenced by both Carl Roger's (1957) client-centered counseling and behavior change theory. Rollnick, Miller and Butler (2008) described MI as clientcentered but with clear goals and direction. A major assumption is that individuals are ambivalent regarding problematic health behaviors. When ambivalence is resolved, change is possible. MI encourages individuals to explore their own reasons for change within a supportive, empathetic environment moving them toward those changes. Behavior change is mediated by intrapersonal conditions which include client change talk and the degree of individual resistance or sustain talk. Change talk is defined as the individual's capacity for arguing for change and therefore acting as a catalyst to motivation. Persuasion or coercion are inconsistent with MI. Client resistance is not 
challenged; rather, it is a cue that the counselor is pushing too hard or using flawed tactics. The focus is on understanding the perspective of the client through reflective listening (Miller \& Rose, 2009). Empathy is used selectively to reinforce client statements. It conveys acceptance, but not necessarily agreement (Rollnick et al., 2008).

The DPP ILI (2002) reported using MI in the original trial. Important to note is the fact that subjects received individual counseling in this trial. Kanaya et al. (2012) reported using MI techniques in a primarily telephone-based ILI with a low income Hispanic population. This was not described or linked to behavioral outcomes. In a meta-analysis of multiple health behaviors, Hettema, Steele and Miller (2005) found significant increases in effect sizes when MI was conducted with minority populations. Limitations include fidelity among facilitators with extreme variability in effectiveness among facilitators (Hettema, Steele, \& Miller, 2005). MI requires significant training with numerous opportunities for practice making it cost prohibitive. MI is meant to guide clients in solving a specific behavior. Again, this contextual aspect is problematic in interventions that target multiple health behaviors. Lastly, MI has been studied primarily through the individual encounter often impractical or non-feasible in low resource community settings.

The behavioral paradigm has been influential in the development of ILI and remains foundational in translational ILI models. The most cited theories or models that provided rationale for ILI are SCT, the TTM and MI. It has been argued that continued emphasis on individual behavior shifts the focus away from social, environmental and structural factors that impact behavior (Chaufan \& Weitz, 2009; 
Krieger, 2001). Individual behavior change in isolation provides a narrow view of solving the extremely complex disparity of diabetes and its prevention in the U.S.

Ecological or Systems Perspective. An ecological perspective was applied in translational ILI in community settings shifting the focus from individual behavior to environmental influences on behavior (McLeroy, Bibeau, Steckler, \& Glanz, 1988). In this broader perspective of health behavior, factors that influence behavior range from the micro- or individual-level to the macro- or structural/ societal-level. Reciprocity is central to an ecological framework; individual behavior influences members of the community; the structural, physical and social aspects of the community have an impact on individual behavior (McLeroy et al., 1988). In contrast to Bandura's conceptualization of reciprocity which views change at the level of the individual, McLeroy, Bibueau, Steckler and Glanz (1988) asserted that public health interventions must focus on changing environments.

Core assumptions of an ecological perspective are that the health and well-being of individuals is influenced by the physical environment such as climate, safe neighborhoods and the social environment such as culture, politics, economics and education. Situational and personal factors also influence health. Situation is defined as place in the social hierarchy, financial resources and living conditions. Personal factors include genetic predisposition and psychological or personality traits/characteristics. Humans react differently to environmental conditions based on these factors. Environments are multi-dimensional; they can be described in terms of physical or social, real or perceived. These dimensions have discrete attributes and cannot be viewed in isolation (Sallis, Owen, \& Fisher, 2008), rather they must be 
considered collectively in relation to their impact on physical, emotional and social well-being (Stokols, 1996). Transactions between humans and their environments occur at different levels: individual, family, work, cultural affiliation, community and population (Sallis et al., 2008). Community-based interventions are proposed to be successful only if they target each dimension across these separate and distinct levels. Lastly, interactions between individuals and their environment are characterized by "cycles of mutual influence" (Stokols, 1992, p 8.). Physical and social environments influence health; health is also influenced when individuals within settings modify the healthfulness of their surroundings through individual or collective actions (Sallis et al., 2008; Stokols, 1996). Social roles and behavior patterns are also influential on the behaviors of others.

Stokols (1996) has referred to his framework as consistent with a social-ecological perspective which pertains to the social, organizational and cultural contexts within the person-environment interaction. Socioeconomic position, employment and education are recognized for their impact on health from the viewpoint of physical and emotional stress as well as for their impact on behavior. The environment is defined by three distinct dimensions: the physical, social and cultural. Each of these dimensions influence physical, emotional and social well-being (Stokols, 1996).

In this review, the social and cultural context was influential in translational ILI for high-risk Hispanic populations. Culture, as a concept, was not defined in the studies reviewed and was not attributed to a theoretical perspective. Translational studies conducted with low-income Hispanic populations described their ILIs as culturally tailored, culturally sensitive, or culturally adapted, often interchangeably. 
These concepts hold different definitions in the literature. According to Kreuter et al. (2003), cultural tailoring is an individual level concept that "(1) is directed towards individuals, not groups; and (2) it is based on known (i.e. measured) differences that exist between individuals" (p.137). Factors that influence individual health behavior should be identified and incorporated into interventions. Resnicow, Soler, Braithwaite, Ahluwalia and Butler's (2000) model for cultural sensitivity in behavioral interventions proposed that both surface structure and deep structure factors be addressed. Surface structure factors include observable social and behavioral factors such as language and food preferences (Resnicow, Soler, Braithwaite, Ahluwalia, \& Butler, 2000). Deep structure factors include the "cultural, social, historical, environmental and psychological factors that influence health behaviors differently across racial/ethnic populations" (Resnicow et al., 2000, p. 274). These are consistent with an ecological perspective. Adapting materials to literacy levels, family involvement, expanding social support networks and integrating cultural values into interventions are examples of deep structure factors (Mier, Ory, \& Medina, 2010).

Cultural adaptation has been defined as "the systematic modification of an evidence-based treatment (EBT) or intervention protocol to consider language, culture, and context in such a way that it is compatible with the client's cultural patterns, meanings, and values" (Bernal, Jiménez-Chafey, \& Domenech Rodríguez, 2009, p. 362). Cultural adaptation is subgroup specific. Barrera (2013) described cultural adaptation as the integration of two divergent perspectives, one being a "top down" approach reflecting a universal view that the intervention applies to all populations and the other a "bottom-up approach" which takes into consideration the "unique 
values, traditions, and practices of a particular subcultural group" (Barrera, Castro, Strycker, \& Toobert, 2013, p.3).

There is conflicting evidence regarding whether adapting for culture has been effective in behavioral interventions in general (Barrera et al., 2013; Castro, Barrera Jr, \& Holleran Steiker, 2010; Mier et al., 2010). Based on this literature review, accommodating for culture was an integral component in ILI in community settings, with some studies exploring social ecological factors in the formative stages of ILI development. Project HEED surveyed community members to explore environmental, social and individual factors that could impact the progression of pre-diabetes to T2D in the planning phase of their study. Findings included a general lack of knowledge in regards to being able to prevent $\mathrm{T} 2 \mathrm{D}$ and poor availability of healthy foods in their neighborhoods. Of those surveyed ( $n=183), 66 \%$ reported that being taught to finish the food on their plates was a barrier to eating in moderation (Horowitz et al., 2011). Barriers to physical activity included time, perception of effort, fatigue, cost and issues related to safety. These findings were consistent with a systematic review of barriers and facilitators of physical activity in Latino men and women (Larsen et al., 2014). In that review, barriers to physical activity in Latina women were related to cultural norms such as putting the needs of the family before their own, cultural attitudes related to the acceptability of exercise and spousal support. They also reported an increased likelihood of exercising if they knew other women who did so and if they had someone to exercise with. Based on the findings of formative research, authors reported "focusing on motivation and education rather than access" in adapting the HEED ILI (Horowitz et al., 2011, p.446). 
Tailoring, modifying or adapting ILI in relation to culture was not empirically linked to clinical or behavioral outcomes in the evidence-based model or in translational ILI in community settings. This was interesting given accumulating literature regarding acculturation theory. Acculturation, defined as "the process by which immigrants adopt the attitude, values, customs, beliefs and behaviors of a new culture" has been associated with some but not all health behaviors related to T2D (Abraído-Lanza, Armbrister, Flórez, \& Aguirre, 2006, p.1342). As an example, obesity, sugar consumption and decreased fruit and vegetable intake were negatively associated with higher levels of acculturation, yet physical activity among Latina women was positively associated (D’Alonzo, 2012; Perez-Escamilla \& Putnik, 2007; Perez-Escamilla, 2011). Acculturation was explored qualitatively for its relation to risk of T2D and to inform ILI development. O'Brien, Shuman, Barrios, Alos and Whitaker (2014) conducted focus groups with a pre-diabetic population of low income Latina women $(n=26)$ with limited access to healthcare. Authors sought to examine shifting roles since settling in the U.S. and the impact of acculturation on lifestyle behaviors. Participants reported a new found ability to afford less healthy lifestyles. For example, they had increased access to and ability to afford sugar-sweetened beverages and fast foods. They could also afford public transportation which prevented them from walking. Participants held the belief that it was their job to ensure the health of their families although it was increasingly difficult to do so due to working outside of the home.

The major theoretical strength of an ecological perspective is that it provides an expanded view of health promotion and disease prevention (Kim, 2010). Similar to a 
systems perspective, people and their environments are dynamic and mutually influential. However, its major strength is also its major limitation (Grzywacz \& Fuqua, 2000). Ecological models that promote health were proposed to simultaneously target each dimension of the environment. This has been criticized as impractical, resource intensive and methodologically challenging (Grzywacz \& Fuqua, 2000). This has lead to a general lack of empirical evidence for using this approach as described by Stokols (1996), McLeroy (1998) and Sallis (2008).

An ecological perspective was used to a limited extent to inform intervention development in translational ILI and to provide rationale for cultural adaptation. ILIs that were informed by this perspective retained the behavioral components of ILI while making what Bandura (2002) referred to as functional adaptations, interventions that are modified for use in diverse cultural settings. Environmental change, a central tenet of this approach, was not an outcome variable or identified as an essential component to ILI in the studies reviewed.

Participatory Perspective. Translational ILI to prevent T2D was theoretically rooted in the behavioral perspective with a focus on individual behavior change. An ecological perspective expanded this view from behavior change initiated solely by individuals. Environmental factors at multiple levels influence health behavior; behavioral strategies consistent with behavior change theories remained foundational in these ILIs. There are several reasons why in isolation these are of limited use. Whether behavior is assumed to be under the control of individuals or the result of environmental influences, the primary aim of these theoretical perspectives is to change behavior. Health behavior is viewed as a universal construct. Tailoring merely 
makes the information more available and acceptable to individuals, yet the actual cognitive and behavioral processes of change are assumed to be inherently the same across populations. As stated by Kim (2002) "psychological models of humans have limiting explanatory use for human phenomena in the nursing perspective" (p.96). Behavior is but one aspect of human living. Social and cultural contexts must be considered not only from the perspective of the patient, but also from the perspective of those who create interventions (Wallerstein \& Duran, 2006). In this respect, the evidence-based ILI is at risk of being a cultural representation of predominantly educated and affluent individuals.

Minkler (2002) has argued that historical and cultural identities are lost when individuals are conceptualized as if they are one in the same. The health of people is dependent on the social context and disparities in health will only be eliminated in participation with those most impacted (Wallerstein \& Duran, 2003). A participatory perspective rejects the positivist research paradigm with a neutral researcher in search of objective knowledge out there. Rather, knowledge is mutually generated or constructed by participants and the researcher.

Participatory research can be traced to two traditions. The Northern tradition has been attributed in part to Lewin's (1946) research on organizational change and action research $[A R]$. Rejecting the prevailing positivist research paradigm, AR was a means to intersubjectively study problems within their natural settings without manipulation or control (Dickens \& Watkins, 1999). The main goal was to not only identify the subjective meanings, values and beliefs that mediate behavior, but to solve practical problems. AR is characterized by cycles of planning, action and reflection (Peters \& 
Robinson, 1984). Problems were viewed as best solved by those who encountered them. Individuals or groups are conceptualized as agents who "choose how they live" after reflecting on problematic situations (Wallerstein \& Duran, 2003, p.30).

The Southern tradition of participatory research is attributed to critical social theorists such as educator Paulo Freire who viewed "community members as subjects of their own experience and inquiry" rather than "communities as objects" (Wallerstein \& Duran, 2003, p.30). Whereas the Northern tradition aims to problem solve, the primary aim of the Southern tradition is to emancipate. Although practical knowledge is generated, individuals or communities create knowledge and come to recognize their own ability to do so. As described by Bradbury and Reason (2003), "knowledge is a living, evolving process of coming-to-know rooted in everyday experience; knowledge is a verb rather than a noun" (p.203). Participatory researchers under this tradition seek to raise critical consciousness and empower community members to transform their own communities.

Throughout its historical evolution, participatory research has been referred to by several terms to include AR, participatory research and participatory action research. The term community based participatory research (CBPR) is consistent with public health research and is particularly concerned with decreasing health disparities (Wallerstein \& Duran, 2006). CBPR has three goals: research, action and education (Wallerstein \& Duran, 2003). Much of the translational ILI conducted in community settings utilized traditional research methods consistent with hypothetico-deductive methods. These were consistent with a power over approach where the researcher is seen as the expert. A major assumption of CBPR is that communities are the experts 
that share knowledge with researchers; together, researchers and communities mutually engage in knowledge production (Wallerstein \& Duran, 2003). The research process is mutually agreed upon between the community and the researchers at all phases to include generation of research questions, research design, intervention planning, implementation and evaluation. These phases often involve the use of multiple methods of data collection.

Participatory approaches were used in translational ILI with high-risk Hispanic populations. Few however utilized research methods consistent with the definition of CBPR (Mau et al., 2010; Ockene et al., 2012; Parikh et al., 2010). Studies conducted using a CBPR approach have used multiple methods to include focus group interviews with high-risk populations to assess perception of risk, inform intervention development and create intervention materials (Horowitz et al., 2011; Kieffer et al., 2004; Makosky Daley et al., 2010).

Perspective of the researcher. This research was theoretically influenced by the social ecological and participatory perspectives with the rationale that: (1) individuals are more than their behavior; environmental factors have an influence on lifestyle behaviors and, (2) individuals and communities are experts in matters that relate to their own health. Advocates of the ecological perspective support the combined theoretical influences of public health, the social sciences, medicine and epidemiology in population health research (McLeroy et al., 1988; Stokols, 1996). In public health, CBPR is increasingly being recognized for its potential in addressing health disparities. In 2007, the CDC's Racial and Ethnic Approaches to Community Health (REACH) program provided funding to support CBPR projects in 22 communities 
across the U.S. In addition, the National Institutes of Health and AHRQ have explicitly called for CBPR in funding announcements for clinical and translational research.

As evidenced in this literature review, the translational research conducted with high risk populations has taken an "all hands on deck approach" (Venditti \& Kramer, 2013, p. 142) by using multiple theoretical approaches to reach high risk populations, specifically ecological and participatory approaches. This is congruent with this researcher's personal conceptualization of nursing: the utilization of multiple theoretical perspectives and strategies to solve difficult problems and improve quality of life. No unifying theory is adequate. Diabetes prevention requires this approach. Multiple disciplines and theoretical influences are necessary.

\section{Conclusion}

In this literature review, the evolution of the evidence-based model to prevent T2D was described. The first diabetes prevention trials sought to determine if T2D could be prevented or delayed with ILI. These were labor intensive and costly. Translational research in community settings required modification or adaptation to make them economical, practical and, in some cases, culturally relevant. Necessary or essential components of ILI for diabetes prevention were cited as behavioral support, behavioral strategies, minimum time and duration, and facilitation by a trained professional. Whether or not these components are essential in translational ILI with high-risk Hispanic populations was not clear.

There was significant heterogeneity in the literature on translation of the evidence-based ILI, with notable trends in the few studies conducted with high-risk 
Hispanic populations. ILIs were more likely to be of shorter dose and duration and be facilitated to a certain extent by CHWs. Social support was provided through individual counseling and group sessions; however, the impact of this was not clear. The most frequently cited behavioral strategies were self-monitoring and goal setting. Self-monitoring in particular was difficult for high-risk Hispanic participants.

Behavioral variables were not consistently measured; studies that measured behavioral variables did not report positive findings in relation to outcomes. In terms of clinical outcomes, weight loss was significant between groups in randomized studies. Weight loss, however, was not consistent with the amount needed to prevent T2D when measured at 12 months. The evidence was not conclusive for weight loss maintenance due to shorter study durations. Physical activity as a behavioral target was unrealized and there was significant variability in the adoption of dietary changes. Culture was an integral component to ILIs conducted with low-income Hispanic populations whether it was defined as culturally tailored, culturally adapted or culturally sensitive. It was not clear, however, whether adapting for culture had an impact on clinical or behavioral outcomes.

Several theoretical perspectives were presented. The evidence-based ILI was conceptually influenced by several psychological theories consistent with a behavioral perspective. Translational ILIs were heavily influenced by the evidence-based model, revealing that a dominant theoretical approach exists within the diabetes prevention literature. The behaviorist perspective reflects the central idea that individuals are in control of their own health and health behavior; the onus of health is placed on the individual (Krieger, 2001). Behavior is a universal construct which can be isolated 
irrespective of other factors. Translational ILIs conducted in high-risk community settings were more likely to integrate ecological and participatory perspectives. These perspectives shift the focus from individual behavior to social and environmental factors that influence behavior and view individuals as experts in matters of health, respectively. The focus remains on behavior; however, these additional theoretical approaches were a means to increase the relevancy of ILI with low income populations. A participatory perspective was applied predominantly with high-risk populations affected by health disparities.

Authors have argued that in order to realize DPP-like outcomes, translation efforts should maintain fidelity to the evidence based model (Venditti \& Kramer, 2012; Whittemore, 2011). Others who reflect a more pragmatic perspective have argued that best practice models such as the DPP do not translate efficiently in community settings (Cohen et al., 2008; Green \& Glasgow, 2006). What works in one community or ideal research condition may not work in those with diverse social, cultural or political characteristics. This argument is central to this research. In the past decade, significant progress has been made in expanding diabetes prevention within the U.S. This expansion, however, has not been universally effective or accessible. Despite translational efforts, a lack of clarity remains as to which ILI components have the most impact on clinical outcomes in high-risk populations historically absent from the research.

Within the diabetes prevention literature, qualitative research methods were used to inform intervention development or explore participant perceptions following an ILI with high risk Hispanic populations. There was an awareness of T2D and its 
complications with less awareness regarding how to prevent the disease. Barriers to nutrition and physical activity in high-risk populations were reported as time, fatigue, money, work, stress and previous unsuccessful weight loss attempts. Facilitators were explored primarily in relation to physical activity with social support identified as an important facilitator. Despite these findings, physical activity was not linked to social support in the reviewed translational studies and physical activity goals were not achieved. Significantly less research was found that explored translational ILI processes qualitatively post-intervention. Parikh et al. (2010) conducted focus groups to explore the perceived benefits and reasons for participating following a translational ILI for high risk African Americans and Latinos. Perceived benefits included eating healthier which impacted the eating habits of the family. Reasons for participating included perception of risk related to $\mathrm{T} 2 \mathrm{D}$ and being persuaded by a trusted individual (Parikh et al., 2010). A limitation of these focus groups was that they were reported within a larger study and it was not clear what was asked or how the data were analyzed. At the time of this review, no studies conducted with high-risk Hispanic populations were found that provided participant descriptions of translational ILI, the influence of ILI components on health behavior change, or the impact of ILI on the everyday lives of participants.

A paradox exists: less is known about populations at greatest risk. This research grew out of a participant observation experience in which a lifestyle intervention program for a high-risk Hispanic population was observed. Although not conceptually influenced by the evidence-based model, preliminary outcomes were favorable. Consistencies with the literature such as physical inactivity were noted, but there were 
also inconsistencies; participants experienced clinically significant weight loss. Although the program clearly reflected a behavioral approach, it did not tightly align with any one theoretical perspective. This researcher, therefore, sought to explore intervention components of a lifestyle intervention from the perspective of the participants who attended the program, identify the perceived facilitators or barriers to making lifestyle changes and explore the impact of a lifestyle intervention program on the lives of a high-risk Hispanic population. 


\section{CHAPTER 3}

\section{METHODOLOGY}

As noted previously, the purpose of this study was to explore intervention components, identify the perceived facilitators or barriers to lifestyle changes within the context of ILI and gain an understanding regarding what is needed to sustain healthy lifestyle behaviors in a high-risk Hispanic population exposed to an existing lifestyle intervention program [LP]. This study was influenced by the lack of exploration of translational ILI from the perspective of the high-risk Hispanic population together with a participant observation experience. During that experience, participants of the LP described the program as life changing. Despite the absence of programmatic factors or components considered essential to prevent $\mathrm{T} 2 \mathrm{D}$, such as professional facilitators or specific weight loss and physical activity goals, preliminary outcomes were favorable for decreases in weight, blood pressure and cholesterol. Further exploration of this LP would clarify whether local programmatic or contextual factors were at work that had yet to be identified in the literature. The research questions posed in this study were as follows:

1. How do participants describe, in their own words, the lifestyle intervention program they attended?

2. What, how and to what extent were participants able to change physical activity and dietary behaviors during the eight week program and sustain those behaviors after the program?

3. What were the facilitators and barriers described by participants in changing physical activity and dietary behaviors and the ability to maintain those 
behaviors after the program?

4. What was the impact of the lifestyle intervention program and individual behavior changes on the lives of participants?

\section{Research Design}

One point of view from which to consider diabetes prevention is from those who stand to benefit. Extant qualitative literature has primarily focused on individual preferences, perception of risk and knowledge regarding T2D prevention to tailor or adapt the evidence-based ILI. Few studies explored individual program components within the context of the evidence-based ILI and its translation for high-risk Hispanic populations. Absent from the literature at the time of this writing were studies in which high-risk Hispanic participants described an ILI for the prevention of T2D in relation to the impact on the lives of individual participants.

This research sought to explore a lifestyle intervention program from the perspective of a high-risk Hispanic population while making few assumptions (Krueger, 1994). Consistent with this aim, this study used a qualitative research methodology taking the position that (1) while an objective physical reality exists, human perception and subjective beliefs about reality vary widely (Phillips, 1990), (2)

there is no uniform means of addressing scientific phenomena; rather, problems can be approached from multiple perspectives using methods consistent with the research questions posed (Shadish, 1993), and (3) the populations for whom health interventions are intended must be part of the solution as well as partners in problem identification (Wallerstein \& Duran, 2003).

A descriptive exploratory design was used for this study. Three focus group 
interviews and one individual interview were conducted with individuals who completed an LP offered by a free urban community health clinic in the northeast region of the U.S. This study initially proposed the use of focus groups alone; however, a modification to the Institutional Review Board [IRB] was granted to include individual interviews when recruitment to groups proved difficult.

\section{Focus Group Interviews}

As a means of qualitative data collection, focus groups provide insight and clarity into phenomena about which little is known, as is the case presented (Frey \& Fontana, 1991; Krueger, 1994; Morgan, 1988). The use of alternative quantitative methods for this problem would lack the perspective and context that may be of significance for future interventions. These reasons, together with the research questions posed, provide the rationale for the method selected.

A hallmark characteristic central to focus groups is the interaction between participants who provide an account of a phenomenon within a social context (Kitzinger, 2008; Krueger \& Casey, 2000). The less dominant role of the researcher in focus groups may decrease the perception of a power differential. Furthermore, the emphasis on participants may be empowering, thus allowing typically disenfranchised

populations to freely engage in discussion and reveal factors not previously considered (Halcomb et al., 2007; Krueger, 1994). The use of focus groups has become increasingly evident with underserved or high-risk populations (Halcomb et al., 2007; Makosky Daley et al., 2010; Rosal et al., 2011; Vincent, Clark, Zimmer, \& Sanchez, 2006).

The traditional positivist research paradigm in which the researcher is viewed as 
expert implicitly creates an imbalance of power (Wallerstein \& Duran, 2006). This research regarded participants as experts of their individual experiences $(\mathrm{N}$. Wallerstein \& Duran, 2003). As such, preparation for this study included building a relationship with the bilingual physician and bilingual CHWs who facilitated the lifestyle program under study. During a prior participant observation experience, a rapport was built over several months between the student researcher and staff members. The researcher shared knowledge regarding diabetes prevention and adult learning principles. In turn, the staff shared successes and challenges with the program. This established relationship between the researcher and bilingual staff members was observed by program participants and may have enhanced participant trust in the researcher. This period of prolonged engagement was instrumental to accurate interpretation of the data enhancing study credibility (Lincoln \& Guba, 1985). For example, during the observation experience, visible frustration was noted from participants when the program did not start on time or when the program agenda was not followed. This was voiced by participants in the focus groups as well. When recruitment efforts proved challenging, the researcher began attending the lifestyle intervention program at weeks six and seven. This was not only to increase recruitment, but also to allow for more opportunities to build trust and increase the comfort of potential study participants.

\section{Description of the Lifestyle Intervention Program}

All participants of this study attended a LP that was offered by the free clinic and facilitated by CHWs either at the clinic or at several community churches. The CHWs attended a 15-week certification program for community health workers. This training 
included topics such as, cultural humility, popular education, leadership and facilitation and community health promotion and capacity building (Community Health Innovations of Rhode Island, 2015). The lifestyle intervention program consisted of 8 weekly sessions. The first session was for pre-intervention data collection which included assessment of blood glucose, blood pressure, cholesterol, waist circumference, weight and knowledge. In sessions 2 through 5, participants received education on topics which included nutrition, physical activity, blood pressure, cholesterol, and diabetes (Buckley et al., 2015). Sessions six and seven were meant to reinforce material and provide social support (Buckley et al., 2015). These last two sessions also included group physical activity. The last session was used to collect data on weight, blood pressure, blood glucose, waist circumference, and knowledge.

The curriculum used was a diabetes education program entitled Thumbs Up® for Health (Oliverio, 2012). A goal of this program was to enhance patient-provider communication and improve health literacy (Oliverio, 2012). The curriculum, designed to be administered over a 4 week period, used pictures to convey messages regarding nutrition, physical activity, a review of medications used for blood pressure and T2D, and education on biopshysical measurements such as blood pressure and blood glucose.

\section{Recruitment and Participants}

This study and all materials used were approved by the IRB of the University of Rhode Island. Permission to conduct this study was obtained by the medical director of the free clinic (Appendix A). Eligibility for the study included having pre-diabetes 
or three or more components of the metabolic syndrome which was identified by clinic staff prior to attending the program. Additional inclusion criteria were: at least 18 years of age, Spanish or English speaking, and self-identified as Hispanic. Participants were excluded if they had pre-existing T2D or did not attend at least four of the eight lifestyle program sessions. During the screening process, one participant who attended fewer than four sessions was excluded from participating in the study.

Recruitment began upon institutional approval of the study and all study materials. Recruitment flyers were placed in several areas of the free health clinic and at community churches where the program took place. The staff of the clinic was provided with a screening binder and instructions on completing the screening tool (Appendix B) in case a past participant inquired about the study when the researcher or research assistant was not present. Recruitment phone calls using the screening tool were made by bilingual research assistants to past program completers. These two recruitment methods proved to be ineffective. As a new LP begins every 8 weeks at the clinic, the researcher began attending LPs prior to the last session to invite those in the program to participate in the study upon program completion. A total of 17 participants were recruited. This resulted in the first focus group interview. Based on the recommendation of CHWs and lack of participation, the researcher continued to attend LPs and conducted the last two focus group interviews immediately after the last session of the LP.

Three group interviews comprised of six, four, and six participants, respectively were conducted. The number of participants in each group allowed ample opportunity for each member to contribute and obtain an adequate range of experiences (Krueger 
\& Casey, 2000; Merton et al., 1990). Three focus groups were consistent with recommendations for capturing patterns and trends between and across groups (Krueger \& Casey, 2000; Morgan, 1988). Interviews were conducted until saturation was achieved, meaning no new information was being offered (Krueger \& Casey, 2000).

The participant of the individual interview and participants of focus group interviews are described in order of occurrence. Participant names were removed from all transcripts and replaced with coded identifiers that were coded in the following manner: P for participant, followed by study number, followed by F or M for gender.

Individual Interview (P1F). This interview was conducted with a 65 -year old woman who was from the Dominican Republic. This participant attended an LP that began in June 2013 and ended in August 2013. Eight weeks had elapsed since the LP she attended ended. She reported attending all 8 sessions of the LP. The LP she attended was facilitated by clinic CHWs but was held at a local church. She was the only member of that particular LP interviewed. The interview was conducted on a Saturday morning when a focus group interview was planned. This participant was the only participant in attendance. After waiting for 30 minutes past the scheduled focus group, the researcher decided to proceed with the interview based on the fact that the participant took the bus to attend and appeared apprehensive about returning for another interview. The participant was reserved at the start, but once the interview began, became increasingly eager to share her story. Initially, the participant avoided making eye contact with the researcher and spoke directly to the moderator who was a research assistant also from the Dominican Republic. As the interview progressed, the 
participant hesitantly responded making eye contact with both the researcher and moderator. This participant was an exemplar case in terms of making dietary and physical activity changes and making an impact on the life of a participant.

Focus Group One (P2F, P3M, P4F, P5M, P6F, P7M). Participants of this focus group all attended the same LP that began in mid-August 2013 and ended in early October 2013. Three participants attended 6 sessions, two participants attended 7 sessions and one participant attended all 8 sessions. The LP ended one week prior to the interview. Recruitment efforts revealed that participants of past LPs did not want to return to the clinic for interviews. This group agreed to attend a focus group one week after their LP ended on the same day of the week and at the same time. This was easiest for them. The group was comprised of three women and three men. Two of the men in the group were spouses of other group members. One couple was native to the U.S., with their parents originating from Puerto Rico. This couple preferred to speak in English, though they did speak in Spanish to other members of the group from time to time. The second married couple was from Mexico. This group interacted positively with one another. There was one male participant who had a tendency to dominate the group; however, it did not appear that other participants were particularly in agreement with him. This was taken into account upon analyzing the data. An interesting finding from this group was the insistence that they did not have the knowledge they needed to be able to make lifestyle changes particularly in regards to healthy eating. The majority of participants in this group was unemployed and attained less than a high school diploma.

Focus Group Two (P8M, P9F, P10F, P11F). Participants of this focus group 
attended the same LP that began in September 2013 and ended in late October 2013.One participant attended 6 sessions, two participants attended 7 sessions and one participant attended all 8 sessions. The interview took place following the last session of the LP. Again, this was due to failed recruitment attempts in having past participants return to the clinic once the LP ended. Members of this group agreed to stay one hour longer following completion of the last session of the LP. This group was comprised of three women and one man. Participants of this group originated from Guatemala. This was perhaps the most interactive of the groups. Statements made by participants often spawned further conversation and lively debate. This enhanced the quality of the data and provided what is referred to by Krueger and Casey (2000) as checks and balances. Two female participants of this group worked together and referred to their relationship in the interview. This group provided the most vivid descriptions of their own motivation or self-will in making dietary and physical activity behavioral changes. They also provided detailed examples of how they overcame obstacles during the program.

Focus Group Three (P12F, P13F, P14F, P15F, P16M, P17F). Participants of this focus group attended the same LP that began in late September 2013 and ended in November 2013. One participant attended 7 sessions. The remainder of participants attended all 8 sessions of the LP. The interview took place following the last session of the LP. The most effective recruitment method became attending the LP at session six and seven to invite participation in a group interview which would take place immediately following the completion of session eight. The group was comprised of five women and one man originally from the Dominican Republic. The interview took 
place in the basement of a community church where the LP had taken place. Of all the interviews, this was the most challenging. The focus group was scheduled on the same day church members, some of which agreed to participate in the focus group, were providing a Thanksgiving dinner for a homeless population. The interview was scheduled to take place on the last session of the LP, which was also the day in which clinic staff obtained all post-program biologic assessments. The assessments were a lengthy process that went past the allotted time. By the time the focus group was initiated, participants were observed to be nervous about the time and seemed anxious to complete the interview. This was felt by both the moderator and the researcher. Of the three groups, participants were more likely to be educated and employed. Another characteristic was the fact that the pastor of the church was a participant of the interview. Although he joined the interview approximately 5-10 minutes after it began, his presence appeared to have an impact on the interaction of other participants. Participants in this group were less likely to provide input for future programs and referred to the LP only positively using terms such as complete. This group was the only to refer to the program in terms of addressing emotional aspects of life in general and in relation to being able to make dietary and physical activity changes.

\section{Setting}

All interviews were conducted at the free health clinic with the exception of one which was completed at a community church that partnered with the clinic. The health clinic provides primary care and preventive health services to a predominantly uninsured Hispanic population. The clinic is located within walking distance for many of its patients and is located near public transit lines. It is staffed primarily by bilingual 
staff members or volunteers with origins from Central American or Caribbean countries. All focus group interviews took place in the late afternoon, as this was the usual time of the LP. The individual interview took place on a Saturday morning. Focus groups one and two were conducted in the conference room where the LP took place for most study participants. The conference room walls were partitioned, which allowed noise from other parts of the clinic to filter into the room. Participants sat at a large oval table that was conducive to conversation. For the first two focus groups, this was the table where they sat while attending the LP. The third focus group was held in the basement of a church where that LP was held. Chairs were placed in a circle for this group. There was ample wall space where research assistants kept lists relating to interview questions. These served as visual prompts in which participants could compare and contrast the responses of fellow participants to their own (Krueger \& Casey, 2000), which added credibility (Lincoln \& Guba, 1985). A healthy snack of fruit, low-fat cheese and water was provided at each focus group interview and at the individual interview. Snacks were well received and eaten prior to and during the interview. Participants took any leftover snacks home with them.

A moderator's guide with semi-structured interview questions informed by the research questions was piloted prior to study commencement with three bilingual CHWs and a bilingual dissertation committee member with expertise in conducting focus group interviews. Modifications were made and the resulting moderator's guide was used to facilitate the discussion (Appendix C). Further modifications were made following the individual interview and the first focus group interview after a debriefing between the researcher and bilingual research assistants (Krueger, 1994; 
Krueger \& Casey, 2000). These modifications were based on the feedback from research assistants and participant responses. Questions flowed from general to more specific and were logically derived from trying to understand the participant experiences and perceptions of the LP (Krueger \& Casey, 2000). At times, participants responded more fully to questions that were direct and specific. As an example, when asked if there was anything that prevented them or got in the way of maintaining new health behaviors, participants responded more fully when they were asked to think about a particularly stressful or bad day since the program ended. Probes for this question included asking them to describe exactly what happened and if this had an impact on diet or physical activity behaviors. This led to the use of this question in subsequent interviews. Focus group interview questions were as follows:

1. We'd like to start by talking about your experiences with the program. Can you please describe the program that you attended?

2. What changes were you able to make while you were in the program?

3. Can you describe any strategies or tips that you learned that helped you make changes in your diet or activity level?

4. Was there someone or something in particular that helped you with making changes?

5. Can you tell us about what your life is like now compared to before the program?

6. Is there anything that prevents you or gets in the way of maintaining the changes you made? Thinking back, can you remember a particularly stressful or bad day that you had? 


\section{Moderators}

All interviews were moderated in Spanish by a bilingual research assistant who was either a bilingual CHW who worked at the clinic or a bilingual senior baccalaureate nursing student with origins from the Dominican Republic. All assistants were compliant with the Health Insurance Portability and Accountability Act [HIPAA] of 1996 and university IRB requirements. Focus groups one and three were conducted by the CHW who was originally from Guatemala. Focus group two and the individual interview were conducted by the nursing student with the student researcher. While it was preferable for the researcher to moderate interviews, use of bilingual moderators was considered for its strengths. The CHWs are trusted members of the community, whereas the researcher represents the larger and sometimes mistrusted dominant culture (Huer \& Saenz, 2003). These bilingual research assistants further enhanced data collection because of their increased understanding of the linguistic and cultural context of participant descriptions (Halcomb et al., 2007). A second research assistant from the Dominican Republic took detailed field notes and provided translation to the researcher when needed. The researcher often initiated further probing of participant responses based on translation from and interaction with the research assistant. The purpose of the field notes was to capture non-verbal communication and any nuances of the discussion potentially missed through audio taping. Field notes also provide an audit trail and lend credibility (Lincoln \& Guba, 1985). Notes included a diagram of the room with a representation of where each participant was located in relation to one another. Less vocal participants tended to sit farthest away from the moderator, while those who spoke freely tended to be closer. In 
some groups, those who sat farther away were quiet initially, but became more vocal as the interview progressed.

\section{Data Analysis Plan}

Each interview was audio recorded in entirety and translated and transcribed verbatim by a native Spanish speaker from Colombia not involved in this research. Transcription was completed as interviews were completed. The transcriptionist had past experience as a research assistant in a federally funded community organization and in transcribing qualitative interviews. Transcripts were precise, meaning that there was no editing of data, such as pauses or interruptions, in order to fully capture what transpired (Krueger \& Casey, 2000; Rubin \& Rubin, 2005). All participant identifiers were removed from the transcripts. Each participant was coded by participant number and gender. Each line of every transcript was then numbered to enable the researcher to easily retrieve text and locate individual quotes.

It is of relevance to note that there is little agreement in the literature in regards to qualitative data analysis. Qualitative research methods represent a range of epistemological, methodological and theoretical perspectives across multiple disciplines which are not amenable to a single approach to qualitative data analysis (Tesch, 1990). This data analysis plan was driven by the purpose of the study and the research questions which sought to explore the experiences of participants. Analysis was further informed by the predominant focus group literature, notably Krueger and Casey (2000). In addition, Sandelowski’s (1995) suggestions for analyzing qualitative data provided guidance. Analysis took into account that focus group interviews are predicated on the interaction of participants, an aspect that is rarely noted in the 
literature (Kitzinger, 2008).

Word counts and pure text analysis were considered inappropriate for the analysis plan for several reasons. Relying on frequency of statements has the potential for the researcher to overlook something of relevance to the research (Krueger \& Casey, 2000). The emotion and intensity in which participants answer questions communicates importance which would be missed with counts or reliance on text (Krueger \& Casey, 2000). Participants represented several Latin American countries and territories. To rely on words alone without context and a degree of interpretation would significantly limit the aims of this study.

\section{Ethical Issues and Trustworthiness}

Considerable effort was taken to ensure that this study was conducted ethically, recognizing that interviews in themselves are interventions (Patton, 2002). Issues of privacy and the potential risk for stressful or emotional responses from participants were considered in the planning of this study and in obtaining IRB approval. The risk to participants was determined to be minimal, as the interview questions were not of a sensitive nature; however, the interactive nature of the study did not guarantee complete confidentiality and privacy. This was clearly stated in the informed consent (Appendix D). Participants were asked to respect one another's privacy. The purpose of the study was explained prior to obtaining informed consent with an explanation of this potential risk. The student researcher took steps to protect the privacy of participants by using first names only during the audio taped interviews. Audiotapes were uploaded to a password and fingerprint protected computer. A back up of audiotapes as well as field notes were stored in a locked filing cabinet in the student 
researcher's office. Once transcripts were received from the transcriptionist, the researcher removed the first names and all identifying information from the transcripts and replaced them with study numbers.

Ethical considerations were also made in relation to conducting research with a potentially disenfranchised population. The concept of cultural humility as described by Tervalon and Murray-Garcia (1998) provided an additional guiding ethical framework. Cultural humility is a continual process of self-evaluation and selfreflection in relation to one's attitudes and beliefs about issues of race, class and social position (Israel, Eng, Schulz, \& Parker, 2005; Tervalon \& Murray-Garcia, 1998). In contrast to cultural competence, which implies an end point, it is meant to be a lifelong endeavor (Tervalon \& Murray-Garcia, 1998).

Cultural humility advocates being alert to imbalances in power and individual biases as well as relinquishing the role of expert (Israel et al., 2005; Minkler, 2005). In this study, the researcher made every attempt to work within the definition of cultural humility. A qualitative research methodology was selected based on the nature of the research questions. The decision to conduct focus group interviews was influenced by their potential for reducing power imbalances and creating a supportive environment for sharing experiences. While the CHWs who assisted with this study did not participate explicitly in its design, conversations with them and their input prior to the study provided further support for the use of focus groups. Requesting assistance from the CHWs who worked at the clinic was not only for translation purposes, but to include a trusted member of the community with whom participants could identify. The established relationship between the researcher and the CHWs may have been 
beneficial in reducing the perception of researcher as outside expert. The CHWs challenged the researcher to look at her own preconceptions and thought processes. For example, CHWs pointed out that the choice in gift cards was impractical for the participants, as the store selected required transportation. Following the first focus group interview, the $\mathrm{CHW}$ research assistant provided the researcher feedback in relation to participant reactions to questions and made recommendations for improving understanding and participation. As an example, questions were originally ordered to inquire about diet-related changes and barriers separately from physical activity changes and barriers. The CHW research assistant related that participants became impatient with these questions when posed separately, as they tended to speak about these behaviors together not in isolation. In time, the researcher came to view these CHWs as the experts.

It has been argued that the criteria for evaluating rigor in qualitative research are not compatible with those associated with the traditional scientific paradigm (Lincoln \& Guba, 1986; Whittemore, Chase, \& Mandle, 2001). Several authors have proposed specific steps to enhance rigor in qualitative research. In this study, steps proposed by Krueger and Casey (2000) and Lincoln and Guba (1986) were taken to enhance quality. Krueger and Casey (2000) state that data analysis is trustworthy when it is systematic and verifiable. The student researcher formulated a data analysis plan prior to beginning analysis and has attempted to be as transparent as possible in describing a detailed account of the analysis process. A detailed audit trail has been maintained and shared with two members of the dissertation committee. Field notes were taken and provided a holistic picture of the focus group process. Consistency across interviews 
was maintained to the greatest extent possible. Modifications were made after the first focus group and remained the same from that point forward.

Trustworthiness has been described as "parallel criteria" to reliability and validity (Lincoln \& Guba, 1986 p. 76), and has been used as a means to enhance rigor in qualitative nursing research (Brown, Wickline, Ecoff, \& Glaser, 2009; Landreneau \& Ward-Smith, 2007; Parsons \& Cornett, 2011; Roy, 2014). Trustworthiness has four criteria: credibility, transferability, and confirmability, and dependability.

Credibility refers to the truth value of a study (Lincoln \& Guba, 1985). The study should be conducted and reported in a manner which renders it believable and steps are taken to actually demonstrate its truth value to the reader (Polit \& Beck, 2008, p. 539). Attempts have been made to thoroughly describe and document all study steps and processes. Cross-checking was completed by using several sources of data. Transcript data were considered in relation to the field notes taken and the lists created with participants during focus group interviews. Member checks were conducted at the end of each interview. The researcher and the focus group moderator ended each interview by summarizing what was said, asking for clarity when needed and ascertaining whether there was agreement from study participants. Each interview was then followed by a debriefing among the student researcher and all research assistants. This was instrumental in further clarification of statements made by participants and ensuring the researcher had as accurate as possible understanding of what was said. Impressions were discussed with the $\mathrm{CHW}$ research assistant who, as a member of the community, confirmed researcher impressions and provided further insight.

Transferability relates to how well the findings may be applied to other settings or 
populations. Lincoln and Guba (1986) proposed that the researcher provide as much thick description as possible. This enables readers to decide for themselves whether or not the findings are relevant to their setting or needs. Every attempt has been made to fully describe the setting, population, study design and data analysis approach.

Confirmability and dependability refer to the idea that, given the same data, findings would be similar or comparable between independent reviewers (Fain, 2013). Two faculty members with expertise in qualitative research independently reviewed the transcript data. Preliminary findings were presented by the student researcher to faculty members. Faculty members and the student researchers came to agreement on coding categories that would be used. The group met again and the student presented a final analysis of themes. Together, the student researcher and the faculty members came to agreement and data analysis was considered complete when consensus was reached. 


\section{CHAPTER 4}

\section{FINDINGS}

This chapter summarizes the findings of the individual interview and the three focus group interviews conducted in relation to the research questions and the categories and themes that were identified.

Data collection occurred between October 2013 and November 2013. Participation in this study was explained as voluntary. Participants were assured that care at the clinic was not related in any way to participation in the study. The purpose of the study was explained fully. Each participant was provided an IRB approved consent form in Spanish, which was read out loud in Spanish prior to obtaining signature. Although the consent was written at a $6^{\text {th }}$ grade level, reading aloud accounted for literacy levels below this level. Consent was obtained for audio recording, which was also explained verbally. Participation in the study was described as being of potential benefit to community members in the future. Aspects of confidentiality were explained. Although full confidentiality could not be guaranteed due to the interactive nature of the study, participants were asked to refrain from discussing what was said outside of the group. Participants were assured that the researcher would maintain confidentiality in regards to their personal information.

\section{Demographic information}

A demographic questionnaire was used to collect name, age, number of adults and children living in the home, preferred language, country of origin, education, employment and date and number of programs attended (Appendix E). The questionnaire was provided in Spanish and English depending on participant 
preference. All materials in Spanish were reviewed for linguistic and cultural relevancy by bi-lingual research assistants. The study was comprised of a purposeful sample of 17 Hispanic adults at high risk for T2D. A homogenous sample characteristic of a focus group methodology was sought; those who completed the LP shared a common experience that was not clearly understood (Krueger, 1994; Merton, Fiske, \& Kendall, 1990). A representative sample of both men and women was sought, though $70 \%$ of the sample consisted of women. This was consistent, however, with the ratio of women to men who attended the LP. Each focus group interview had one married couple, which was unintentional. Participants were of similar socioeconomic position. At the time of this study, lack of health insurance was the eligibility requirement to receive primary and preventive care services at the clinic. Participants represented several countries of origin, including the Dominican Republic, Guatemala, Mexico and the U.S. The two participants from the U.S. had parents who originated from Puerto Rico. Consistent with the literature, each focus group had no less than six and no more than twelve participants which is optimal for interaction and discussion (Krueger, 1994).

The preferred spoken language of participants was Spanish, with the exception of one married couple who were bilingual and chose to communicate with the moderator and researcher in English. Of the 8-week LP, 47\% reported attending all 8 sessions, $29 \%$ attended 7 and 24\% attended 6 sessions. Demographic information of the sample is detailed in Table 1. Table 2 provides further breakdown of focus group and individual interview characteristics. 
Table 1

Description of the Sample

\begin{tabular}{|c|c|c|}
\hline Variable & $n$ & $\%$ \\
\hline \multicolumn{3}{|l|}{ Gender } \\
\hline Female & 12 & $70 \%$ \\
\hline Male & 5 & $30 \%$ \\
\hline \multicolumn{3}{|l|}{ Age } \\
\hline $18-29$ & 1 & $6 \%$ \\
\hline $30-39$ & 3 & $18 \%$ \\
\hline $40-49$ & 7 & $41 \%$ \\
\hline $50-59$ & 5 & $29 \%$ \\
\hline$>60$ & 1 & $6 \%$ \\
\hline \multicolumn{3}{|l|}{ Country of Origin } \\
\hline Republica Dominicana & 7 & $41 \%$ \\
\hline Guatemala & 6 & $35 \%$ \\
\hline Mexico & 2 & $12 \%$ \\
\hline U.S. & 2 & $12 \%$ \\
\hline \multicolumn{3}{|l|}{$\underline{\text { Preferred Language }}$} \\
\hline Spanish & 15 & $88 \%$ \\
\hline English & 2 & $12 \%$ \\
\hline \multicolumn{3}{|l|}{ Employment Status } \\
\hline Unemployed & 7 & $41 \%$ \\
\hline Part time employment & 6 & $35 \%$ \\
\hline Full time employment & 3 & $18 \%$ \\
\hline Item left blank & 1 & $6 \%$ \\
\hline \multicolumn{3}{|l|}{ Highest level of education } \\
\hline$<$ HS diploma & 8 & $47 \%$ \\
\hline HS diploma/equivalent & 3 & $18 \%$ \\
\hline Some college & 1 & $6 \%$ \\
\hline College degree & 2 & $12 \%$ \\
\hline Item left blank & 2 & $12 \%$ \\
\hline \multicolumn{3}{|l|}{ Interview Size } \\
\hline Individual interview & 1 & $6 \%$ \\
\hline Focus group one & 6 & $35 \%$ \\
\hline Focus group two & 4 & $24 \%$ \\
\hline Focus group three & 6 & $35 \%$ \\
\hline
\end{tabular}


Table 2

Description of individual and focus group interviews

\begin{tabular}{|c|c|c|c|c|c|}
\hline & & $\begin{array}{l}\text { Individual } \\
\text { Interview }\end{array}$ & $\begin{array}{c}\text { Focus group } \\
1 \\
N=6\end{array}$ & $\begin{array}{c}\text { Focus group } \\
2 \\
N=4\end{array}$ & $\begin{array}{c}\text { Focus group } \\
3 \\
\mathrm{~N}=6\end{array}$ \\
\hline \multicolumn{6}{|l|}{ Gender } \\
\hline & Female & 1 & 3 & 3 & 5 \\
\hline & Male & & 3 & 1 & 1 \\
\hline \multicolumn{6}{|l|}{$\underline{\text { Age }}$} \\
\hline & $18-29$ & & & 1 & \\
\hline & $30-39$ & & 1 & 1 & 1 \\
\hline & $40-49$ & & 3 & 2 & 2 \\
\hline & $50-59$ & & 2 & & 3 \\
\hline & $>60$ & 1 & & & \\
\hline \multicolumn{6}{|c|}{ Country of Origin } \\
\hline & Guatemala & & 2 & 4 & \\
\hline & Mexico & & 2 & & \\
\hline & Republica & 1 & & & 6 \\
\hline & Domenica & & & & \\
\hline & U.S. & & 2 & & \\
\hline \multicolumn{6}{|c|}{ Preferred language } \\
\hline & Spanish & 1 & 4 & 4 & 6 \\
\hline & English & & 2 & & \\
\hline \multicolumn{6}{|c|}{ Employment status } \\
\hline & Unemployed & 1 & 4 & & 2 \\
\hline & $\begin{array}{l}\text { Part-time } \\
\text { employment }\end{array}$ & & 2 & 3 & 1 \\
\hline & Full-time & & & 1 & 2 \\
\hline & Item left & & & & 1 \\
\hline & blank & & & & \\
\hline \multicolumn{6}{|c|}{ Education } \\
\hline & $<\mathrm{HS}$ diploma & & 3 & 3 & 3 \\
\hline & HS diploma & & 2 & & 1 \\
\hline & Some college & & 1 & & 2 \\
\hline & $\begin{array}{l}\text { College } \\
\text { degree }\end{array}$ & & & & \\
\hline & $\begin{array}{l}\text { Item left } \\
\text { blank }\end{array}$ & 1 & & 1 & \\
\hline \multicolumn{6}{|c|}{ Number of LP sessions attended } \\
\hline & 6 sessions & & 3 & 1 & \\
\hline & 7 sessions & & 2 & 2 & 1 \\
\hline & 8 sessions & 1 & 1 & 1 & 5 \\
\hline
\end{tabular}




\section{Findings from Interviews}

Each group interview began with informal introductions and a pre-session activity unassociated with the interview topic to make participants comfortable (Krueger, 1994). A pre-session activity also allowed the researcher to get a feeling for participant characteristics that could impact the interview. For example, participants who were reserved or quiet as well as those who were more outgoing were noted. The pre-session activity involved going around the table and sharing with the group a custom or tradition that was special to them and included the bilingual moderator, assistant and researcher. At the end of each interview, a summary of what was discussed was provided by the student researcher based on the field notes and communication with the moderator. This gave participants the opportunity to provide further clarification and add any content not covered (Krueger, 1994; Morgan, 1988). Each interview lasted approximately one hour. Participants received a gift card in appreciation for their time in the amount of $\$ 15$. This was increased to $\$ 20$ after consulting with the CHWs and in response to poor interview attendance.

The data was analyzed by the student researcher and consisted of four stages. Transcript data was approached in an inductive data-driven manner (Sandelowski, 1995; Tesch, 1990). In the first stage, the student researcher immersed herself in the transcripts by reading each in entirety to get a general sense of the data as a whole (Sandelowski, 1995; Tesch, 1990). Each transcript was then re-read separately several times through making notations in the margins and highlighting or underlining pertinent phrases. This allowed the researcher to gain an impression or sense of each interview individually. 
In the second stage of analysis, the student researcher began to organize the data based on the research questions. This initial method of creating coding categories or an initial framework was intended as a means to organize the data into a workable form while remaining open to emergent categories (Sandelowski, 1995; Tesch, 1990). The decision to create a code was based on four factors: frequency, specificity, emotion and extensiveness (Krueger \& Casey, 2000). When the same comment is said frequently, it lends credibility; however, an important finding may only be said once and should be considered within the context of what is being explored (Krueger \& Casey, 2000). Specificity relates to the degree of detail used by participants in providing description. Comments that are made with intensity or emotion may reflect importance or relevance. Extensiveness refers to how often a comment is made by a single participant. For example, one participant in this study was adamant about not having the information. This began to have increasing weight when he repeated this throughout the interview.

Four notebooks were used, one for each interview transcript. Preliminary coding categories were placed within each notebook. Specific text or participant comments which related to the coding category were added to individual transcript notebooks as reading and coding proceeded. Decisions were made on whether comments fit within a coding category or were more representative of a new category or sub-category. Each time a new coding category was identified, it was placed in all four notebooks. In doing so, an audit trail was maintained which revealed the extent in which a particular code was identified in an interview. The student researcher could then easily review each notebook to compare and contrast interviews. Notebooks also allowed for 
reflective journaling throughout the analysis process and note taking.

A project was created using the qualitative analysis software, NVivo 10. Transcripts removed of identifiers were uploaded and nodes were created for each code. Codes were then placed within a parent node for each research question. NVivo was used primarily as a tool to identify trends in participant descriptions based on demographic information, such as gender. As an example, several participants voiced the desire to share their stories with others. This was voiced only by female participants.

In the third stage of data analysis, each interview transcript was color coded and re-printed on colored paper maintaining line numbers. The color coding enabled the researcher to visually discern which group the coded quotation or text came from while also seeing the extent in which a particular coding category or sub-category represented the sample as a whole. Referring back to each transcript notebook, quotations from the text which represented a coding category or sub-category were cut from each transcript and placed in a pile. In cutting quotations, care was taken not to alter the context of the text or disrupt a segment of conversation between participants which supported or refuted a comment.

Large poster paper was used to display each coding category and sub-categories with its corresponding color coded text. Moving systematically, the student researcher went through a process of carefully considering each code in isolation, its relation to other codes and adequacy in addressing the research question. This was the lengthiest stage, as the researcher constantly reconsidered each code and referred back to the research and interview questions, participant comments and the field notes. As this 
stage of data reduction progressed, some codes were eliminated and others were subsumed within other coding categories.

In the fourth phase of analysis, the student researcher and two dissertation committee members with experience in qualitative data analysis met to review coding categories and the themes identified for each research question. This final phase included stepping back from the data. Going into data analysis, it was important to this researcher to avoid focusing on words alone. An important aspect of data analysis is the ability to reflect on the individual groups and their interactions as well as certain facial expressions, conviction in tone, and the boisterous and the reserved. Data analysis was considered complete when there was consensus among the student researcher and dissertation committee members and all research questions were answered. The resulting coding categories and themes are represented in Table 3. 
Table 3

Coding Categories and Description of Themes

\begin{tabular}{|c|c|c|c|}
\hline$\frac{\text { Research }}{\text { Question } 1}$ & 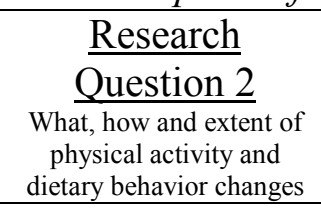 & $\frac{\text { Research }}{\text { Question } 3}$ & $\frac{\text { Research }}{\text { Question } 4} \underset{\begin{array}{c}\text { Impact of LP on lives of } \\
\text { participants and on individual } \\
\text { behavior }\end{array}}{ }$ \\
\hline $\begin{array}{l}\text { We learned } \\
\text { We learned what } \\
\text { we didn't know }\end{array}$ & $\begin{array}{l}\text { Rapid adoption } \\
\text { of new dietary } \\
\text { and physical } \\
\text { activity behaviors }\end{array}$ & $\begin{array}{l}\text { Facilitators of } \\
\text { healthy diet and } \\
\text { physical activity } \\
\text { Physical and } \\
\text { emotional benefits } \\
\text { Support from } \\
\text { family and friends } \\
\text { Persistence }\end{array}$ & $\begin{array}{l}\text { I like how I am now/ } \\
\text { I don't want to go back }\end{array}$ \\
\hline $\begin{array}{l}\text { We learned new } \\
\text { information } \\
\text { They would tell us } \\
\text { and show us } \\
\text { Specific strategies }\end{array}$ & $\begin{array}{l}\text { Linking health } \\
\text { behaviors to } \\
\text { health outcomes }\end{array}$ & $\begin{array}{l}\text { Barriers to dietary } \\
\text { and physical } \\
\text { activity } \\
\text { Physical sensations } \\
\text { Social and } \\
\text { emotional aspects } \\
\text { of daily life } \\
\text { Unawareness of } \\
\text { healthy food } \\
\text { options }\end{array}$ & $\begin{array}{l}\text { I am conscious of what } \\
\text { I need to do }\end{array}$ \\
\hline They helped us & $\begin{array}{l}\text { Self-regulating } \\
\text { through cognitive } \\
\text { processes }\end{array}$ & & $\begin{array}{l}\text { I want to keep } \\
\text { going/continue forward } \\
\text { Individual changes in } \\
\text { lifestyle behavior has } \\
\text { affect on others }\end{array}$ \\
\hline
\end{tabular}




\section{Research Question One: How do participants describe, in their own words, the}

\section{lifestyle intervention program they attended?}

The aim of this question was to explore participant descriptions based on a gap noted in the literature in regards to program components and preliminary favorable outcomes of a lifestyle intervention program for a high-risk Hispanic population. Descriptions in the words of participants were sought to gain insight and clarity into the lifestyle intervention program and its components. Participants were asked to describe the program they attended. The majority of participants responded enthusiastically to this question. If prompting was necessary, participants were asked what activities they did or what they learned while in the program. Before moving on, participants were asked if anyone experienced something different or if wanted to add anything not mentioned. Participants described the program in the following manner: (1) We learned what we didn't know, (2) We learned new information and (3) They helped us.

We learned what we didn't know. When asked to describe the program, a prominent finding was the way participants described receiving information of which they were generally unaware. Out of the four interviews, just one participant spoke of receiving prior education regarding diet or physical activity in the past, noting that "they never gave it to us in such details." Not having the information was adamantly described, particularly by participants in focus group one, yet remained a common thread throughout the interviews. One example was:

Before, like I told you, I used to have an uncontrolled life in the fact that I did not have an education on how to eat, I did not walk, I did not exercise, a life 
like that. Everyday more fat, more fat. (P1F)

When probed whether they would have made dietary or physical activity behavior changes on their own, several participants responded by saying: "No, we didn't have the information," "We didn't have the information to do the things right or wrong for us" and "We didn't know nothing about this."

Participants also described having the perception that they were already eating healthy or being physically active only to find out that there was a significant amount of information that they did not know. For example, one participant stated, "According to me, I used to eat a lot of vegetables, but I eat a lot of rice. According to me, I was eating healthy, vegetables, chicken, tortillas" (P6F). Another participant stated, "I wanted to lose weight but I never was able to until I came here," and still another, "At your own house, you don't know what you have to do."

We learned new information. The majority of participants began their sentences in the beginning of interviews with "I learned" or "we learned" and progressed into detailed accounts of what exactly they learned. In describing the LP they attended, participants used terms such as educational, interesting, and helpful. Across all interviews, participants began with descriptions about what they learned in relation to eating a healthy diet. The most common dietary recommendations that were named included increasing fruits and vegetables, decreasing fat and salt and increasing daily water intake.

They would tell us and show us. Participants were exact in describing how they learned the information while in the LP. The information they received was very specific and practical. For example, they were given instructions for exactly which 
milk to buy. This was recounted as factual information provided through two primary formats: lecture and visual power point slides. In describing the program one participant responded:

Look, the program was like this. They would explain to us point by point and we would be noting. For example, they would tell us these have more sodium, these have less, or sugar, these have less. I am sick of [high blood] pressure. I maintain it really high, so then they would say, "For the people that are sick of high blood pressure, for people who are sick of high cholesterol, or people that are diabetic." So, they would tell us like that. Explaining themselves well, very nice, I really liked it. (P8M)

Another participant stated:

For me it was very pretty and very interesting because well, I met more people and they told me a lot how, what you should eat, what you can do, and the most important is that they help you be more motivated to do it. To be able to live healthier. (P9F)

Program materials were described as being highly visual and interactive which accommodated low literacy proficiency. Power point slides showed pictures in which participants could actually make visual comparisons of food labels, blood pressure readings or physical activities. One teaching method described by participants was thumbs up for healthy foods or biologic measurements such as blood pressure and thumbs down for less healthy options or measurements. One participant explained an exercise used with the aid of PowerPoint slides:

One of the exercises that was done twice was, thumbs up, thumbs down [visual 
slides]. Thumbs up was if it was good for us and thumbs down was if it was bad for us. Then they would put two images and they would tell us, for example the regular Coca-Cola has so many grams of sugar and the diet CocaCola has so much sugar. So then, they would say what's good for me? So, you would have to know if it's A or B. (P11F)

Another participant related seeing pictorial representations on slides were helpful: If it wasn't for the healthy living program, I would probably weigh 250 to 260 pounds because every day I would weigh more and more. After I went to the program, and they would put it on the screen and everything, watching how people that eat fats and those that don't eat fat, and looking at people that exercise and that don't exercise, comparing one thing with another, well you take conscious. (P1F)

Specific strategies. Participants described being taught specific strategies to assist with dietary and physical activity behaviors. An example of this was being taught to make small incremental changes. For example, when asked how they were able to make dietary changes, one participant commented:

To lower the quantity of food little by little. For example, in Guatemala we eat tortillas. If we ate three tortillas start eating two instead. Lower it little by little and go from there to not eating it. If we eat a lot of rice, eat less rice. And, try to diminish the quantity of carbohydrates. (P9F)

This same participant described being told to monitor her food intake:

They would give us a piece of paper and you had to write down what you ate from Monday through Wednesday and the good thing is they said not to lie. 
So, then you start noticing that you have to write the truth and I liked that they did that because you start to realize the quantity that you're eating and everything that you're eating daily. I liked the program to be honest. The truth is that I liked it. I liked it a lot. (P9F)

Another strategy mentioned by multiple participants was that of decreasing carbohydrate intake and eating carbohydrates with other foods. Participants referred to this as "balance." For example, one participant noted:

You cannot call it a diet, do you understand? Because they never tell you to stop eating but to learn to balance out the food. I liked it a lot and if they were to do it again and they accept me, I would like to do it again. (P11F)

Another participant commented:

I think it has been of great blessing because we have educated ourselves about portions also and to balance the food. If we eat carbohydrates we also have to add vegetables-the salads and try not to drink sweetened drinks but to drink more water. (P17F)

Participants were also taught ways to increase physical activity, such as integrating easy to perform activities throughout the day. Participants described learning to park their cars further, walk up and down the stairs while talking on the phone and to use the stairs instead of elevators or escalators. Participants described being taught that physical activity did not mean needing to go to a gym. One participant described being taught to use household items to perform strength training:

When you are at the house watching TV you could grab a bottle of water like this and do this [flexing arms] or with soap like Tide. You bend down and you 
do like this [stretching arms out to sides]. There are many ways to do exercise without spending money, she said. You can do it at your house. (P9F) This discussion took place among participants commenting about being physically active:

(P13F): We have also learned that for us to exercise you don't need specifically to go to the gym. It is good for those who can, but for those who can't, can do it at our house while we watch TV, while we do laundry, we can go up and down the stairs.

(P12F): The same thing. I'm with (P13F), that we learn to park farther...take time to do the exercises at home even if it's 30 minutes or 20 minutes whatever but to take out time.

(P13F): I say the same thing, you guys that this program is so complete. You brought the exercise. They helped us a lot. In every session, we had diverse exercises.

(P14F): We learned to educate our bodies, move our bodies, to exercise, we can exercise another way at home without having to go to the gym (P13F): We did it (physical activity) in a very dynamic way because we got it playing around. We sweated and had fun with other people.

They helped us. Participants described the community health workers who facilitated the LP using words such as helpful, motivating and encouraging. In addition, although many participants revealed personal goals for attending the LP were to lose weight, they described program facilitators encouraging them to be healthy rather than focusing on weight loss. This was described by one participant who 
described an encounter with the CHW who facilitated the program:

She spoke with me and she advised me to give me motivation and told me to put a lot on my part. That even if something would come across my mind to eat or not to go exercise, always think primarily of my health. And that motivated me a lot. (P11F)

Participants described being instructed about what they needed to do to be healthy and relayed the belief that they could not have done so without the help of facilitators. Program facilitators were described as motivators for change. This was not only in teaching the material, but also in providing direct one-on-one support to program participants. Motivation provided by program facilitators was described as merely being present, speaking with participants and presenting the material. As an example, when asked about whether someone helped them make diet and physical activity changes, participants interactively described support from program facilitators:

(P10F): For me it was [two program facilitators] because they would give us advice and would motivate us a lot continue. And also [a third facilitator]....she would talk to you and she would tell you, "Do this, this is good." She would give you advice.

(P8M): Like a support.

(P11F): And she would encourage you to come next Wednesday. They say, "Come next Wednesday."

(P8M): Yes, she [third facilitator] would always say, "welcome" they would say and on Tuesdays at 4 o'clock they would leave us a message.

\section{Research Question Two: What, how and to what extent were participants able to}


change dietary and physical activity behaviors during the eight-week program and sustain those behaviors after the program?

This research question corresponded with questions two and three of the moderator's guide. In interviews, participants were asked specifically what kind of changes they were able to make, without making a distinction between dietary and physical activity behaviors initially. Participants eagerly listed the dietary changes they were able to make; however, as interviews proceeded it was noted that most participants reported making dietary and physical activity changes simultaneously. Participants also described methods they enlisted to make changes.

Rapid adoption of new dietary and physical activity behaviors. Participants described being able to implement new dietary and physical activity behaviors very quickly. When one of the groups was asked to make a list of the things they were able to change during the program, one participant exclaimed, "Oh my God, a lot of food. A lot of food!" There was significant focus on the sodium content in foods with many participants describing how they reduced their sodium intake simply by learning how to read food labels. The addition of fruits and vegetables was noted frequently. One participant stated, "I started eating fruits, I started eating vegetables. Do you understand? I started walking, doing exercise. I walk 25 to 35 minutes daily. Every day" (P1F).

Once aware, participants described little resistance to adopting the new behaviors. For example, one participant stated:

There are things that I was unaware of. I'm telling you, the second class that we were here I was already making changes. Like, one was sodium, I didn't 
know that sodium was salt... So, now I'm looking at the label and making sure that it doesn't go over the limit of sodium. The day they told us in the second class was the same date that I changed all my food. (P2F)

Another participant commented on his ability to maintain new dietary changes immediately following the program:

Well, for me, in the week that we haven't seen each other, I have continued with the same: cooking without so much salt, and I'm reading with more desire the books that you gave us. I'm learning more from those books, reading what cereals you can consume more because in the book it's more specified and you can see it more detailed instead of you coming to this class. So, every night I grabbed the book and I've read it. (P8M)

Most participants described eating smaller portions, reducing sodium intake and drinking more water which was done by flavoring with fruit. Many participants discussed eating more salads without describing the contents of salads. Participants stated that the program they attended did not reflect a "diet;" rather, the focus was on decreasing portions a little at a time. Multiple participants, however, also related eliminating certain foods entirely from their diet. For example, they eliminated bread, sugar-sweetened beverages and fast-foods.

Linking health behaviors to health outcomes. Participants were able to make connections between dietary and physical activity behaviors and certain health outcomes such as hypertension, high cholesterol and weight. One participant described being hopeful that new physical activity behaviors and weight loss during the program would have an impact on liver enzymes that were tested every six months. In the 
period immediately following the LP, participants described noticing changes and linking them to a behavior, which created a favorable condition to sustain those behaviors. For example, while replying to a question about changes that were difficult to make, one participant described in lay terms how blood pressure is affected by excessive sodium intake by stating:

[In English] For me, it was the salt. Yes, because if you get a handful and you put on the food and you take the pressure, it's going to be high. Now if you put less or a little, your pressure is going to be regular and your heart is going to be pumping more blood so it gives you more movement. (P3M)

This participant later stated:

Eating the way that we eat now, we feel like a brand new person, like a brand new life to us and it's like a gift you gave us...We saw people with problems, with cholesterol, with heart problems and now when you see them they don't have anything. (P3M)

Another participant described the realization that not only did diet and physical activity lead to weight loss, but the behaviors also have an effect on blood pressure and cholesterol:

I was able to lower my cholesterol, my arterial blood pressure normalized, I learned that you have to combine exercise with food...because it's not about losing weight it's also to maintain the arterial blood pressure lower and the cholesterol and how to do exercise to lose weight. (P7M)

Participants described a prior belief that diet and physical activity alone did not have the potential to improve health outcomes. This was described by a participant 
who first told of not receiving medication for his hypertension and high cholesterol, but progressively linked the implementation of new health behaviors to favorable health outcomes: "When they told me that I was sick, they didn't give me any medication. The only thing I had to do was exercise, exercise, exercise" (P8M). And then:

What it is, is the food. So first, I had cholesterol high and they didn't give me medicine they just told me that the diet, the food and all that and they didn't give me anything. So, I started to check the books and all that and the chats really helped me. I like that a lot, I liked it and like P $9 \mathrm{~F}$, if there was another program, I would participate. (P8M)

Later in the interview, he describes his decision to incorporate physical activity: I would see a man walk every day. He would walk and I would tell my other friend, 'This guy, he's really dumb, he should rest.' And now, I told my friend let's go for a walk. First, we would walk and then we would run. Yes, it was hard, but I was able to do it and now I run one mile, two miles and I don't feel it. (P8M)

Lastly, the man reports, "I have lost 14 pounds. My cholesterol was really high. I was at 290 almost and now I have it at 170.”

Self-regulating through cognitive processes. Several participants described instances when they stopped to think about what they were eating. "Control," "selfcontrol" or "self-will" was used multiple times across interviews when participants described how they were able to make or sustain changes. In the previous section, participants described being instructed to write things down as a beneficial strategy for 
monitoring food intake; however, participants also described instances when they regulated their behavior through self-talk, processing what they were doing prior to actually doing it:

I had a goal before starting the healthy living class. I had the goal to lose weight, to do exercise, all that. But since the program, I am more motivated. Now every time I go to eat something that is not good for my health, I think, "Wait a minute, I have to get better." (P14F)

And another participant noted:

There is always obstacles because there are temptations when you're used to your entire life to eat something and in a moment you say, 'No, no I can't eat that because that is harmful to me,' but you try little by little for your benefit to do it. (P1F)

\section{Research Question Three: What facilitators and barriers are described by participants in changing dietary and physical activity behaviors and the ability to maintain those behaviors?}

As a whole, study participants were more likely to discuss facilitators of eating behaviors or physical activity rather than barriers. Questions four, five and eight of the moderator's guide addressed this research question.

Facilitators of healthy dietary and physical activity changes. Participants identified two primary facilitators for making dietary and physical activity changes: (1) experiencing the physical and emotional benefits and (2) support from family and friends.

Experiencing the physical and emotional benefits. Participants described 
experiencing physical and emotional well-being that served as facilitators. Observing change in one's self was described as a facilitator to changing and maintaining newly adopted health behaviors. These changes were both physical changes such as weight loss and increased energy levels as well as a change in affect. Weight loss and energy were attributed to both physical activity and healthy eating and served as both facilitator and motivator for maintaining those changes. For example, the participant from the individual interview described her increased tolerance for physical activity and attributed it to her weight loss:

I would get too tired doing Zumba. And that's what I would do. As time went by I got used to more exercise, sweating, and then I would walk around the park with my friends that were also doing the course. Every day, one hour....Then I started to feel like I could tie my shoe and I was losing weight. And you start getting excited and I continue to do my routine daily. (P1F) When this same participant was asked what has helped her to maintain healthy eating she responded, "How I feel. I feel very good thanks to the program...Before I used to eat a lot and I started to eat a little. Now I feel better. Now I cannot eat a lot because I feel good"(P1F).

A focus group one participant also maintained new health behaviors based on how he felt physically:

Before I came here I got scared because of all the things that I have, cholesterol, high blood pressure, I was like a walking heart attack person....now I don't have none of that. So, I feel good in and out. You know because cooking the proper way, we're eating less, drinking a lot of water, a lot 
of fruit you know so it's like a pick me up. I mean before I used to be sluggish and now I'm not sluggish. I am alert. I can do a lot of things-things I couldn't do before. We feel lighter, you know? (P3M)

Following up on this comment, another participant from the same interview commented:

For me, now I feel more lively, like with more energy. I don't know...I feel like even though I've always done exercise, I feel calm like, I don't know. It was a change like I have more encouragement to do more things. (P5F)

Support from family and friends. Social support from other group members, friends, partners and CHWs who facilitated the LP was described as a facilitator to eating well and increasing physical activity. Participants described being supported by other participants in the LP. This was in the form of direct moral support and was described indirectly. For example, one participant who attended the LP with a coworker who was also in the focus group described being chided about what she was eating:

There were times that my friend would look at me with a plate of food and she would ask me, "You're going to eat that?" And that would be kept in my conscious, "Oh my God, but I'm eating this." Seriously, because we work together. Then, yes, I can say that she is helping in that sense to control myself because sometimes you wanted to eat more than enough. (P9F)

Social support between friends who completed the LP together was described in terms of eating healthy and being physically active by a participant:

My friends helped me a lot. We get together and go walking at the park and 
when we come from walking, we eat something healthy. And they help me like I help them and we talk to each other, "We are not going to drink this because this has so much sugar" or "We are not going to drink that because it has so much carbohydrates." The one that called me right now. You see how we check up on each other? (P1F)

A female participant from group two described how her husband, who did not attend the LP, supported her at home:

My husband, he has helped me a lot....now we eat so differently. He also eats the same thing and he does exercise. He put a machine, like a bicycle, so we can have it at home. He started doing exercises with me and he helps me a lot. In response to this, a female participant sitting across the table probed, "You mean he doesn't make you lasagna anymore?" Her response, "No, he makes some but I take it to work for my co-workers" (P9F/P11F).

During the focus group one interview, there was considerable discussion on the topic of social support. The moderator asked a female participant who had been very quiet if there was someone who helped her implement lifestyle changes. She replied, "I live alone with my child so what helped me was the group. The team here that was with us, they helped me a lot and the group, they helped me a lot" (P10F).

Barriers to making dietary and physical activity changes. When participants were asked what type of barriers they encountered in making dietary and physical activity changes during and immediately following the program, participants described: (1) physical sensations, (2) social and emotional aspects of daily life and (3) unawareness of healthy food options. 
Physical sensations. When asked what gets in the way of eating well or being physically active, participants described physical sensations that posed challenges such as hunger and fatigue. One participant described hunger and the sense that something was missing when decreasing portion sizes:

What made it difficult was the fact that my stomach was used to getting more food.... What was difficult was the first days to get my stomach to get used to eating less food. So it was then that I was hungry, but you feel a way kind of like something is missing, a little bit more. I don't know if you understand me, but to see a pretty plate full and then to change, what can I tell you? To change the rice for the salad and little quantity of rice that's what you mostly eat, what you like the most that made it difficult for me. So, the quantity because the stomach is used to eating a lot. (P9F)

Another participant commented on hunger, "For me, it was [difficult] to eat less because before I would eat more... At first you kind of stay hungry but later its best to make an educated self. But that [hunger] was a little hard" (P6F). In regards to being physically active, two participants discussed how fatigue could potentially pose a barrier by stating:

(P11F): Because of tiredness.

(P9F): Because when you come from working, all tired, what you want to do is lie down on the couch and watch TV. After you come to your house, you know, take care of the kids and do house chores and after that go do exercise. That is really hard because that requires a lot of self-will.

(P11F): A lot. To do exercise has been the hardest thing. The hardest decision 
I've made in my life, to start to do Zumba. Yes, I get very tired at work and then I come and do exercise.

Social and emotional aspects of daily life. Many participants found social situations where food was abundant as challenging. Participants described how they overcame these situations. When asked to describe a day that was difficult one participant responded, "every day," while another stated:

Yes, my love, every Sunday...because it's a day that you share with the family. For example, me at church, I have a lot of friends that invite me to eat every Sunday. Sometimes I have three invitations and I would like to go to all three of them but I have to choose one. (P11F)

When probed to describe more about being in these situations and how they were handled, the participant commented:

I first thought of my health. And when it was time to eat, I wouldn't eat whatever. I always tried to eat salad with grilled chicken breast. Nothing fried, all grilled. I always took care of myself. (P11F)

Making dietary changes that involved reducing the intake of foods associated with cultural heritage was difficult for participants. One participant with origins from the Dominican Republic described how it was difficult to give up certain foods:

The hardest one to leave, the fat, the fried food, that's what I like the most. That was like a sacrifice that I did. Like when you have something that you like and you have to leave it...it was the fried food, French fries, McDonald's. It was eat fried food like all Dominicans, salami, fried green plantain. You know, all the food that I always said I couldn't stop eating and eat what I'm 
eating now. (P1F)

Another female participant originally from the Dominican Republic stated:

Some of the traditions that we do have in our culture is that we eat a lot of rice.

It's like rice, rice, rice.... We eat a lot of rice and it's hard for us to reduce the quantity of rice now that it is a carbohydrate and all. (P15F)

A third participant from the Dominican Republic followed up with:

In our culture we eat a heavy breakfast. In the morning you can see somebody eating mangu, they could be eating yucca, or something heavy in grease. Our foods are not healthy. Also, for lunch we have a heavy food. And, also dinner is a heavy plate... Yes, yucca, eggs, salami, grease. Culturally, we are badly informed about nutrition. Thank God and thanks to the clinic we have learned a lot about it. (P13F)

Lastly, another participant noted, "It was hard not to eat fat, because we are Mexicans and Mexicans like fried food" (P7M).

Emotional stress was described as a barrier to eating. When faced with a particularly stressful day, participants reported they were more likely to eat less fruits and vegetable or foods that were not good for them. This was particularly true for several participants in focus group three who worked at the church where the LP was held. One of these participants described "dedicating more time" to themselves and went on to say:

Sometimes with stress, the life that we have, so accelerated, we tend to stop taking care of ourselves. And, we don't eat on time. So, to think a little bit more of ourselves and to be a little bit more careful to eat at the right time. 
(P16M)

Another participant in this focus group interview associated stress and distressing emotions to eating behaviors:

It [the program] has been very important, a great blessing that it has created a consciousness of the things we can do to take care of ourselves. Not just adapting because, oh, it's the age, it's the metabolism, I can't deal with this. Just give up to stress. Because stress sometimes makes us eat more than what we should. (P13F)

Lastly, participants simply described everyday hassles as getting in the way of being physically active:

It's not a prevention, [obstacle], it is that sometimes you have an appointment. For example, we have an hour let's say at six [to exercise], but sometimes you have something very important like go with the son to an appointment or your own appointment. For example, I work at home and sometimes I have a lot of work and I can't do it. (P6F)

Yet, when asked to describe how they would improve the LP, most participants suggested more than eight sessions. One participant voiced a willingness to attend stating, "Because one you're in the program, you will be modifying your appointments" (P4F).

Unawareness of healthy food options. Across interviews, participants voiced a desire to continue new behaviors after program completion. There was a residual unawareness of healthy food options, however, which posed a potential barrier to sustaining dietary behavior changes. For example, these participants made these 
comments after already being specifically asked about barriers:

(P2F): Can I say something? There is food out there that we are unaware of, what's good for us.....I know there's a lot of food out there that we could cook that's good for our bodies but I'm unaware of that. I don't know what kind of foods they are.

(P4F): Yes, for example, for breakfast, I always think, "What other thing is out there that I can make that's healthier?" And, I don't know. I try to make something different every day.

Another participant provided further support for this unawareness while she was making suggestions for future programs:

Yes, it was good, but if you would have added some examples, it would be better to learn things, like sometimes there are things that you just don't know how to cook. Then it would be good that they tell us how to cook it, meaning an example. Because there are some vegetables that we are like, "What's that, how do you cook that?" (P6F)

Persistence. This last category emerged from the manner in which participants reported barriers and could be interpreted as an actual facilitator of behavior changes. When participants described barriers, most were quick to note that they did not give up and were persistent in their attempts to eat well or be physically active. They often described how they overcame barriers or related their disappointment when hectic schedules stood in the way of being physically active. Physical activity in particular was described as being the most difficult to change, yet participants rarely gave examples of being unable to perform the behavior. 
When they spoke of situations in which they experienced setbacks or felt discouraged they also spoke of starting over rather than abandoning efforts. For example, this participant described a setback he experienced while attending a social event:

I confess that I did lose the diet because there was desserts, pork shoulder, everything. I said to myself only a little but I had no control. Everybody was eating. It was the fourth week [of the program], but well, I started all over again. What I did was I went home and did my shake of celery and peppers. (P8M)

A second participant acknowledged that while his wife and he were not always able to eat the way they knew they should, they should keep trying "to put it in practice:" [In English] I can say we're starting to keep trying because we learn we got to continue to do it, to make the goal, you know?...Many times we leave the house early and we have a day very agitated and very difficult by doing a lot of stuff. It doesn't give us time to sit down to drink something...it is not something we want to do, we have been used to this but we are getting better and we continue to get better. I can say that. We are doing better. (P16M)

Participants described their own motivation as being the driving force in changing lifestyle behaviors and persevering. One female participant from focus group two poignantly stated:

Nothing can be achieved if you don't put your part because one, one can have the disposition, well, there is the want and the does. And, if you don't do it, then, you can't do it. (P11F) When this same participant was later probed about 
how she dealt with a difficult social event she stated, "O love, at first I would give up but now I don't. I try to eat by portions" (P11F).

\section{Research Question Four: What was the impact of the lifestyle intervention program and individual changes on the lives of participants?}

The aim of this question was to explore ways in which the LP impacted the daily lives of participants. This research question was addressed with question six on the moderator's guide; however, much of this research question was answered throughout interviews as participants described their experience during and immediately following the LP.

I like how I am now and I don't want to go back. One way in which the LP had an impact on participants related to being satisfied with themselves after making dietary and physical activity changes. One said;

After the program, I feel healthier, I exercise daily, and I eat a little like you're supposed to eat. I eat a lot of salads because before I did not eat salads. Now, you go to my fridge and it's filled with broccoli, cauliflower, lettuce, cabbage, carrots, you know, things like that. And before, I didn't do that. I didn't even look at it....Yes, I maintain all the time. I maintain my rhythm of life that same way. I have not started to eat fat, no, it does not provoke me now. Pork meat, I have not eaten it anymore. Only white meat, fish and I feel good. (P1F) Another participant stated, "I like to be healthy. You know, back then I did not know about healthy and now healthy and eating the proper way is the best way it is" (P3M). This was perhaps most poignantly expressed by a participant who said very little and often needed to be encouraged to join the discussion. When the moderator was 
inquiring about barriers to maintaining behavior changes, she replied simply, "I feel better this way and I don't want to change for anything" (P4F).

I'm conscious of what I need to do. Many participants of the healthy living program reported an increase in consciousness or a new awareness which had an impact on them during the program or in the short period following the program. Once participants had an awareness of a healthy diet or ways in which they could be physically active, they voiced the inability to ignore these facts. For example, one woman reported:

I was talking to a friend about this program because you eat, and I'm sorry to say this you eat like a pork [pig]. It's only grab and eat and eat, you are not seeing how much salt, sodium, sugar you are giving yourself...Now, when I go buy something I check. I read how much it has of sodium, how much of it has sugar and it has helped me a lot. (P9F)

The participant from the individual interview made this revealing statement:

I have changed so much, that it does not call my attention, the meat, the pork skin, pork meat, things like that with fat, none of that calls my attention. None of that gives me a desire. I got used to eating healthy. That food doesn't call me anymore. McDonald's, I have not eaten that anymore, or Burger King or any of that. It does not call my attention. (P1F)

This increased awareness did not always lead to positive feelings in relation to eating well. When asked to describe a day that was difficult in relation to eating well or being physically active, two participants related:

(P15F): For me it was yesterday, because I was very busy....I was not able to 
have breakfast. I mean, I have breakfast really late. I had dinner very late. So, I didn't really feel, like this morning I didn't want to come, because I knew that even though the effect is one day, it really affected me because I didn't eat right and it's because I got out of the daily routine.

(P13F): Well, I could say the same thing, too. Last night we left here late too and we had a full packed day. Then we got home and I ate things that I understand are not healthy. So, there is now a consciousness and many times, now we feel bad when we fall off what we normally eat healthy.

I want to keep going/continue forward. Participants described a significant educational gain while being left wanting more. Many participants relayed that they would either repeat the program over again or believed there should be additional sessions. This was described as having momentum with the desire to "continue moving forward." This was particularly true in the case of physical activity. The majority of participants indicated that more sessions of the LP should include organized physical activity. Participants spoke of an organized Zumba class that was starting on a weekly basis at the free health clinic with enthusiasm and in a manner that suggested it removed an element of decision making. Attending an organized dance class would satisfy their goal of adding physical activity.

Participants wanted to continue as well as have the opportunity to make up sessions they may have missed, as stated by a participant:

I had been trying to lose weight for a very long time and I was able to do it here in a month. I lost 15 pounds and that has me very motivated and I want to continue forward. I want to continue to participate in future programs if they 
give us the opportunity to attend the sessions that we didn't attend that would be better. (P6F)Another participant specifically commented, "The program motivates you to continue moving forward." (P9F)

A third participant from focus group two voiced her desire to learn more about nutrition stating:

I would like to learn what vitamins are in vegetables and fruits. I would like that to be explained because sometimes you eat but you don't know what vitamins it has. I know that the oranges, the lemon and the mandarins have vitamin $\mathrm{C}$ but the vegetables. (P9F)

Lastly, one participant simply stated, "We learned a lot...a lot was mentioned but we don't know if there is more." (P5M)

Individual changes in lifestyle behavior has affect on others. Participants described how newly acquired knowledge and the adoption of lifestyle behaviors had an impact on other family members as well as friends. One participant stated:

I feel very well...I already knew a little bit but I have learned so much more regarding how to cook and feed myself, how to feed my son and I have a little bit more consciousness. Now with my child he says, "Well, you can't eat that." If he asks for more food, [I say] "no, that's enough, you already ate." I am trying to take better care of him and get better. And I feel very happy, yes. $(\mathrm{P} 13 \mathrm{~F})$

Another participant in this group described how the LP has had an impact on how she cares for her husband who has T2D:

I also learned more because my husband is diabetic...to help him how to eat, 
how to feed himself, the portions, the food. I live controlling him because he likes to eat a lot of fatty food so now he says, "Now you are going to put me in control [of T2D]" and thank God, he has the T2D under control. (P14F)

The participant from the individual interview described how implementing dietary changes has had an impact on her entire family:

You know a lot of people go to my house. I have a lot of friends and family and grandchildren. I have 12 grandchildren and 4 great-grandchildren and they go [to my house] because I used to make a lot of food like pork shoulders, brown beans, a lot of food, you know, food like that. To not cook like that anymore a lot of people have to adapt to eating salads and to eat stew and to eat baked chicken without the skin. It's harder for them because there's no fat. (P1F)

\section{Additional Findings from Interviews}

In this section, additional findings are presented. These findings did not align directly with the research questions, yet were deemed valuable because they provided additional clarity in regards to whether additional components are needed in lifestyle interventions for high-risk Hispanic populations. Additional questions that were asked were (1) Some people have asked for the LP to continue. If you could create the LP, how would they be? What would you suggest? How would you like to receive the information? And, (2) If you did not attend all 8 sessions of the LP, can you tell us why? Other findings presented here were said with sufficient frequency, specificity or intensity they earned their way into the findings and were considered relevant to this research. Three additional findings were categorized as: (1) participant 
recommendations for future programs, (2) persuasion enhances program participation and (3) give testimony.

Participant recommendations for future programs. One of the last focus group questions was, "If you could create a program, what would it be like?" Interestingly, though the majority of participants had praise for the program, when asked this question, they had multiple suggestions for improving the program. Recommendations fell into three sub-categories: dose/duration and content, structure and teaching methods/learning preferences.

Dose/Duration and Content. The majority of participants felt the program was not long enough in duration, with several participants stating they would attend the same program over again as a means to prolong the program. For example, one participant stated, "Unfortunately, time is not enough and there is a lot you need to learn in this little time. I find that it's a little time" (P7M). Another participant added, "It was eight weeks long, I think we need more. Because it looks like those eight weeks was crunched up of topic after another topic just to complete in two hours" (P2F).

Several participants suggested that, if the program was longer, then more educational content could be added. Participants asked for additional content such as learning about vitamins and minerals, more examples of vegetables and how to cook them and recipes for healthy meals.

Participants overwhelmingly requested more opportunities for physical activity, especially Zumba, a form of dance lead by program facilitators. Zumba was described as "dynamic" and "fun." The topic of Zumba generated lively discussion in focus 
group two who wanted access to Zumba outside of the program in addition to within the program:

(P6F): I would like more Zumba.

(P4F): You need to include in the program.

(P6F): I think that if they would've given us in the program the Zumba...

(Moderator): More Zumba?

(P6F): Yes, because we only did one class and if it would've been combined we would've lost more weight.

(P5M): Yes, combine it, one hour of class and one hour of Zumba.

(P4F): Meaning, yes combine it.

(P3M): I can't wait until they open the Zumba class because there are a lot of people that I know...I saw them on Saturday and I said, Wow! At the Zumba class.

(P2F): There would be a lot of people.

Participants were also open to other types of physical activity to which they had not previously been exposed. For example, when probed further about how they would change the LP, several participants from focus group two responded together:

(P11F): In those sessions you could really add more than two sessions of Zumba. Because we have two, out of the eight they are two Zumba classes. So, you could put more.

(Moderator): Anything else, or may be something that you didn't do in the program?

(P10F): Maybe like yoga. 
(P9F): Never in my life have I done yoga. I don't even know what it is.

(P10F): A class of yoga that would be good. We could learn a little bit more. (P8M): But not just Zumba, another exercise. Do you understand?

(P10F and P9F together): Another type of exercise.

(P9F): Yes, because I know that some men don't like to dance.

When asked why they did not attend all 8 sessions of the program, three reasons were identified. Participants stated they were ill and could not attend or that they had previous engagements. Participants also revealed that they were allowed to join the LP after it began. These participants joined the LP at the second session. Participants did not attribute missing the class due to transportation or child care.

Structure. Participants commented that that at times, program content overlapped from week to week and felt that if there was less overlap there would have been more time for Zumba. For example, a participant noted:

(P6F): Well for me, I don't know about you guys, everyone has their own opinions, right? For example, if one day you have a topic try to finish the topic in that day. The next week, have another topic. Meaning don't repeat the same topics. No, because then you lose time in repeating yourself. Like have a topic one day and finish it that day.

(P4F): Like have a schedule.

(P6F): Meaning I would like that but maybe not everybody.

(P2F): No, that's good.

(P4F): Me too.

(P6F): We would learn more... because we are following along but it gets 
tiring.

(P4F): And it loses its importance.

Participants also noted that they were looking forward to a celebration that was planned at the end of the program but did not come to fruition. This was voiced with disappointment and spoke to their desire to celebrate as a group:

(P7M): It would also be good to have a topic per day and would be more organized. One thing that we would like, well that I would like is that the graduation would be how they said that it would be. Like everybody would bring a different plate.

(P2F): Yes.

(P7M): Like we felt that was missing, to coexist. The last one.

Additional teaching methods/learning preferences. In terms of receiving the material, participants requested additional methods to enhance their learning. Some examples included being given homework and materials for note taking. Two participants had this discussion which eloquently demonstrated different learning styles and their preferences:

(Moderator): There are many ways to learn the material and to be taught. Is there another way you would like to learn the material?

(P11F): Like for them to give us homework. Because for example, I am the type of person, we all have different capacities to learn things, some people are visual, so reading, others are audio, and others are writing. I learn faster, for example, I learn faster and I retain more if I write. If I read and write. (Moderator): Okay. 
(P11F): So if they would leave us homework, for example, let's say 3 to 5 questions of what we learned, respond to them and bring them to the next class.

(P9F): Yes, it was well done how they did it, but like (P11F) said, we don't all have the same capacity of capturing everything in the mind. So, it would be good that they would let us write. For example, they could explain it to us and we could write it so we could practice later. Because at least me, all the things about the (blood) pressure in the moment I knew it, but if you ask me now, I don't know everything.

When further probed if a pamphlet would be helpful the one of these participants responded:

No, not that I take home, more that I write it so I can understand it better because they give us a book. They gave it to us and it explains everything. But as you write it, you will understand more. (P11F)

One participant indicated that written materials were beneficial but were not a replacement for in-person instruction and contact with program facilitators: "If you give me the paper I could read in Spanish but if the person is in front (of you) it's even better because they can motivate you" (P1F).

Persuasion is an effective recruitment strategy. Multiple participants referred to being convinced, invited or persuaded to attend the LP and spoke of a program that essentially came to them. For example, a participant described:

I remember the moment that they invited me to come and I used to say no, no, no. I used to think to myself I do my exercises at home, I don't need it. But at 
the end they convinced me to come and I don't regret it (P6F).

While one married couple stated:

(P3M): Yes, she [the program facilitator] was the one that saw us sitting down and said, 'You guys have to go to a class about healthy eating.' (P2F): We said yes.

Still another participant stated, "To me this has helped me a lot. Well, since I got sick and they invited me to this class...If there was another program I would participate" (P4F). The manner in which this was described suggested that they were surprised by being invited to attend the program. This could be as a result of a genuine unawareness of the program, but could also reflect the novelty of someone seeking them out and taking an interest in their health. For those without access or very limited access to healthcare, this would perhaps be unexpected and could also be considered from the perspective that participants were unaware that programs such as the LP existed. When the participant from the individual interview was asked whether she would attend more sessions, she described asking program facilitators if she could attend another program, she stated, "but they didn't call me," further indicating this sense of needing to be asked personally to attend.

Give testimony. The LP that participants attended was eight weeks in length. Many participants were eager to attend either additional sessions or the program in entirety again. In each interview, participants voiced the need for others to receive the information that they had received. As an example, the participant of the individual interview stated:

I have changed so much that it does not call my attention. The meat, pork skin, 
pork meat, things like that with fat, none of that calls my attention....The thing is that I have lost weight. My life has changed so much and that helps other people to continue to do what I did. I would like that. I would like to give my testimony of what I've done.(P1F)

This participant went on to say:

I would like there to be a lot of people [at the program], especially to be able to share with all the people in one session and motivate them so that the people can do the same thing that I did. Do you understand? (P1F)

Another participant stated:

Yes, it [the LP] was very educational. Everyone should be aware of it. I mean, I know that there are a lot of people that are unaware of it and we should move on and tell others about the program because it is educational. We are aware of it, we lost pounds, we lost weight, you know? And, it does work. (P2F) In this chapter, the findings were presented in relation to how participants of a lifestyle program intended for a high-risk Hispanic population describe that program. Participants described the LP they attended as a novel learning experience that provided practical and detailed information. Participants described the program in the following manner: (1) We learned what we didn't know, (2) We learned new information and (3) They helped us. Participants described the ability to rapidly adopt multiple changes simultaneously by linking behaviors to health outcomes and selfregulating through cognitive processes. Participants identified two facilitators to making behavior changes: (1) physical and emotional effects and (2) supportive family and friends. The concept of persistence emerged as a category when participants 
described making dietary and physical activity behaviors in general and in relation to overcoming barriers. The barriers to dietary and physical activity behavior change were described as biophysical sensations and social and emotional aspects of daily life. A third barrier, unawareness of healthy food options was identified specifically in relation to the ability to sustain dietary behaviors. Lastly, participants described the impact of the program they attended in the following ways: (1) I like how I am now/I don't want to go back, (2) I'm aware of what I need to do, (3) I want to continue forward/keep going and (4) individual changes affect others. Additional findings included participant recommendations for future programs. These were related to (1) dose/duration and content, (2) structure and (3) teaching methods/learning preferences. Participants also voiced their desire to share what they learned with others.Despite not being tightly aligned with the evidence-based model, these findings indicate that participants of the lifestyle program benefited from components that were consistent with that model. 


\section{CHAPTER 5}

\section{DISCUSSION AND CONCLUSION}

This chapter will discuss the results of the study in relation to the research questions posed. The limitations of the study will be presented followed by the implications for nursing research, education and practice. Lastly, a summary of the study will be presented.

\section{Research question one: How do participants describe in their own words the}

\section{lifestyle program they attended?}

Participants described their experience in the LP as beautiful, excellent, magnificent, informative and, helpful. The overarching category or theme in relation to this question was articulated simply as, we learned. Several important findings in relation to this research question will be discussed which include knowledge acquisition, in-person support, and dose and duration.

Participants had the perception that they were already eating a healthy diet and to a less extent, being physically active. Upon attending the lifestyle program, they realized that in fact, there was information in which they were unaware. Participants described a general lack of awareness regarding what it means to eat healthy as described by one participant who stated, "We didn't know nothing about this." Similar findings were reported in the literature in relation to eating well and awareness of T2D risk (Kieffer et al., 2004; Rosal et al., 2011). The lack of awareness regarding dietary and physical activity practices may be attributed to several factors. Participants described unawareness in terms of the information simply not getting to them. Belonging to a community in which public health initiatives such as diabetes 
prevention programs have not reached them may be a factor. This could be due to the availability of programs in the preferred language. As was the case in the literature, a paucity of translational ILIs were conducted with high risk Hispanic populations suggesting that a scarcity of community programs also exists. Across interviews, however, there was no mention or reference to the inability to access healthcare or disease prevention or health promotion programs. This was despite the fact that all participants in this study lacked health insurance.

Another factor may be due to health care practices. It was noted in the literature that Hispanic men and women were more likely to seek health care in acute circumstances (Ai et al., 2012; MacNoughton, 2008). This may contribute to the lack of awareness regarding preventive practices. Even when insured, minority populations do not receive the same quality of healthcare (IOM, 2003). It is possible that this population has not received the caliber of lifestyle counseling and patient education as more affluent populations in the primary care setting.

The idea that participants were unaware of healthy lifestyle practices because of inaccessibility or lack of quality preventive services was supported by their surprise at being offered the LP. Participants were invited or convinced to attend by CHWs, spouses and friends who attended the LP. In-person verbal persuasion either by a trusted community member, family member or friend was a noted recruitment strategy in the literature (Santoyo-Olsson et al., 2011; Parikh et al., 2010; Horowitz et al., 2009).Lastly, surprise at being offered the LP may have been due to an unawareness of risk of T2D. Perception of risk was influential in the decision to participate in translational ILI for high risk Hispanic populations (Santoyo-Olsson et al., 2011). 
Participants identified the provision of practical and factual knowledge as an essential component of the LP. This is consistent with essential criteria of ILI identified in the literature and evident in translational ILI in community settings and with high risk Hispanic populations (Knowler et al., 2002; Whittemore, 2011; Ali et al.,2012;Horowtiz et al., 2011; Ruggerio et al., 2011; Merriam et al., 2009). Going "point by point" suggested that the information was given concisely and simply. This was found to be an effective educational strategy in the literature as well (Mau et al., 2010; Ockene et al., 2012)

An essential component of ILI for the prevention of T2D was in-person support from a facilitator (Knowler et al., 2002; Venditti \& Kramer, 2012: Ali et al., 2012). The findings of this study support the need for this component. Participants felt that having in-person instruction increased their motivation. There was disagreement in the literature in regards to the level of expertise one should possess to deliver ILI for the prevention of T2D (Venditti \& Kramer, 2102; Ali et al., 2012). Studies conducted with underserved or low income populations most often used CHWs to facilitate ILI (Kanaya et al., 2012, Mau et al, 2010; Ruggiero et al., 2011;Seidel et al., 2008) and have been found to be as effective as those utilizing professional facilitators (Ali et al., 2011). The CHWs in this study were described as knowledgeable experts.

In this study, participants partially attributed their ability to change to CHW program facilitators voicing doubt in initiating changes on their own. CHWs may have provided the encouragement and feedback required increasing participant confidence or self-efficacy. Feedback as a strategy was an important strategy in the evidencebased IL (Knowler et al., 2002). Although linking self-efficacy to support from CHWs 
was not noted in the diabetes prevention literature, $\mathrm{CHWs}$ were identified as trusted members of the community who share similar experiences and understand cultural and contextual factors that impact the adoption of healthy behaviors (Ockene et al., 2012; Horowitz et al., 2011; McClosky, 2009). Participants may have viewed CHWs as role models which can also increase one's confidence in their ability to perform the behavior (Bandura, 2004).

Behavioral strategies were an integral component to ILI for the prevention of T2D. In this study, the most prominent behavioral strategy was implementing small easy changes. This strategy has been reported in translational ILI with high risk Hispanic populations (Horowitz et al., 2011; Delgadillo et al., 2010). Small achievable goals were considered more important than study outcomes in one study (Delgadillo et al., 2010). This is consistent with Bandura's (1977) Self-efficacy Theory in which it is proposed that mastery of a behavior strengthens efficacy expectations or the expectation that one can successfully execute the behavior required to produce the outcomes of the behavior (p.193). This was supported in this study. Participants described being motivated by their accomplishments voicing the desire to maintain new behaviors perhaps because increased confidence in their ability to do so.

Research question two: What, how, and to what extent were participants able to change dietary and physical activity behaviors during the eight week program and sustain those behaviors following the program?

Discussion points in relation to this research question include rapid adoption of multiple behaviors and physical activity. 
Rapid adoption was a prominent finding in this study. Participants described changing dietary behaviors, in particular, almost immediately upon receiving the information. This was not identified in the literature. This may be related to several factors. The novelty of the information may have influenced behavior. The concept of empowerment is a process in which individuals gain mastery and improve quality of life (Minkler, Wallerstein \& Wilson, 2002, p. 289). Participants in this study may have felt empowered by the information they were receiving and felt an increased sense of control over their health. The program may have increased their perception of risk of T2D which has been associated with the decision to make behavior changes (Rosal et al, 2011).

Adoption may have also been influenced by previous independent weight loss attempts that were unsuccessful. Kolb's Experiential Learning Theory (2005) proposes that adult learners change when they have experienced a challenge and can apply what is learned to that experience. This may further explain the ease with which participants were able to make changes and lose weight in the LP. Lastly, as previously noted, the strategy of making small, easy changes was beneficial. This may have overshadowed behaviors that participants found more difficult. Comments such as "doing the exercise that was the hardest decision I've ever made" or referring to a full plate as "pretty" suggest that not all behaviors were adopted with the same ease and speed.

It is of relevance that newly adopted behaviors drop off or decrease over time (Diabetes Prevention Program Research Group, 2009). A maintenance phase of up to one year was the most effective means of preventing this (Knowler et al., 2002). Translational ILI in high risk Hispanic community settings reported positive dietary 
behavior changes but often lacked a maintenance phase (Ruggiero et al., 2011; Ockene et al., 2012, Horowitz et al., 2011; Kanaya et al., 2012). As was the case with those studies and with this one, it cannot be determined whether or not participants will maintain new behaviors in which they initially adopted with ease. It may be that over time, dietary and physical activity behaviors will taper off as evidenced by studies that included a maintenance phase (Katula et al, 2013).

The literature recommended 150 minutes of physical activity per week. This was a goal in the evidence-based ILI for the prevention of T2D. Translational ILI with high risk Hispanic populations inconsistently applied this goal (Ruggerio et al., 2011; Horowitz et al., 2011, Ockene et al., 2012; Kanaya et al., 2012). Translational ILI with high risk Hispanic populations that set this goal did not achieve it (Ruggiero et al., 2011; Horowitz et al., 2010). The LP in this study did not have explicit physical activity goals. It was notable however that many participants reported making physical activity changes. Although this was self-reported, this should be considered in relation to the literature which consistently reports a sedentary lifestyle or lack of leisure time physical activity in the Hispanic population (Larsen et al., 2014; Neighbors et al., 2008; Marquez \& McAuley, 2006).

Despite reporting independent physical activity, the majority of participants requested additional group exercise opportunities. There was considerable excitement regarding regular Zumba classes being offered at the free clinic. This may suggest that performing and sustaining new physical activity behaviors hinges on providing organized activities. This was supported in the literature. Translational ILIs conducted with a low income Latino population that did not provide organized physical activity 
found no meaningful improvement in physical activity at one year (Ockene et al., 2012). Studies that provided physical activity for Hispanic populations most often offered dance which was referred to as culturally appropriate (Horowitz et al., 2011). In this study, participants enjoyed Zumba and requested additional classes however participants were also willing to try other forms of physical activity. Men in this study also requested physical activity other than dance which was consistent with literature (Marquez \& McCauley, 2006). These data suggest that preferences for physical activity should not be assumed and tailoring may be beneficial.

Participants described changing dietary and physical activity behaviors by linking behaviors to health outcomes. This was consistent with Horowitz et al. (2004) who found that low-income African American and Latino participants did not fully appreciate the impact of diet on hypertension. In this study, there was prior disbelief or doubt that diet or physical activity was potent enough to improve or eliminate health problems. This is a particularly salient finding given the beneficial effects these behaviors have on insulin sensitivity and blood glycemia.

The evidence-based ILI supports the use of behavioral strategies such as selfregulation which includes concepts such as self-monitoring, goal-setting and problem solving. Self-monitoring was identified in this study primarily through cognitive processes such as pausing to consider their dietary options and using self-talk. Selfmonitoring was also identified based on feedback received from influential others such as other group members. This is consistent with Social Cognitive Theory (2004) which proposes individuals weigh the expected benefits and costs related to performing a behavior and that behavior is partially influenced by the reactions it generates in 
others. Individuals regulate their behavior by directing their attention to the behavior, reflecting and self-evaluating (Bandura, 2004). This study supports self-regulation as a potential mediator of dietary and physical activity behaviors in high risk Hispanic populations.

\section{Research question three: What were the facilitators and barriers described}

\section{by participants in changing physical activity and dietary behaviors and the}

\section{ability to maintain those behaviors after the program?}

In this study, participants were more likely to talk about facilitators rather than barriers to dietary and physical activity behaviors. The literature revealed fatigue, time, motivation and access as barriers to physical activity in Hispanic populations (Larsen et al. 2014; Rosal et al.; 2004; Rosal et al., 2008) .This was supported only minimally in this study. Even with probing, participants were insistent that was little that would get in the way of maintaining new behaviors. Although participants identified fatigue and hectic schedules as barriers to physical activity, the majority of participants who identified these barriers were quick to report overcoming them. Participants in this study did not relate that they were unable to be physically active due to the physical environment.

Physical and emotional effects experienced as a result of making dietary and physical activity behaviors facilitated continued performance. Parikh et al. (2010) reported that participants felt they benefited from new dietary behaviors in postintervention focus groups but this was not related to physical or emotional effects. The physical and emotional effects of physical activity was not noted in the diabetes prevention literature reviewed. In this study, physical effects included increased 
energy and ability to perform daily activities of life. Emotional effects were described as feeling "calm." This may reflect the stress reducing benefits of physical activity. This study found that social support from family and friends was an important facilitator of dietary and physical activity behaviors. This was consistent with the literature. In terms of dietary behaviors, support from family members was an important facilitator among Latina women (Ramirez, Chalela, Gallion \& Velez, 2007). Support from family and friends were also positively correlated with physical activity (Larsen et al., 2014; Marquez \& McCauley, 2006).In this study, physical activity was most often described as being performed with others such as spouses or friends. The group format of the LP was a facilitator with participants commenting that it was the group that helped them in general. This is consistent with a translational ILI with a high risk Hispanic population which reported that fellow group members were instrumental to reaching individual goals (Parikh et al., 2010).

A noted barrier to dietary behaviors was experiencing biophysical sensations such as fatigue and hunger. Fatigue was a common barrier to physical activity in the literature (Rosal et al., 2011; Marquez \& McCauley, 2006). Horowitz et al., 2011 found that being raised to clean their plate was a barrier to healthy eating in a low income Hispanic population. In this study, participants described decreasing portion sizes and resisting the urge to fill their plate. This may have attributed to hunger. Participants stated that at first, this was difficult suggesting that in time they became accustomed to eating less. In this study, eating poorly was related to emotional stress which was reported in the literature (Dallman, 2010). 
Preferences for cultural foods that were known to be high in fat and carbohydrates were identified as a barrier to eating well. When participants described foods as part of their cultural experience, they used words such as unhealthy, heavy, grease, fat and fried. Participants expressed an interest in unfamiliar foods evidenced by one participant asking, "What other thing is out there that I can make?" Teaching how to make food preferences healthier may have more of an impact on maintaining healthy eating behaviors, particularly because participants described an emotional attachment to some of their favorite foods. A healthier version of these foods has the potential for providing that sense of familiarity without removing it entirely. This has been reported in translational ILI with high risk Hispanic populations (Ockene et al., 2012) and was supported in this study by requests for a Hispanic cookbook with healthy recipes.

Women in this study were equally eager to be physically active commenting, "We take time to do the exercises at home even if it's 30 minutes or 20 minutes whatever but to take out time," and "We did it in a very dynamic way because we get it playing around. We sweated and had fun with other people.'This is in contrast to findings in which the construct of marianismo was a barrier to physical activity in Latin American women (D'Alonzo, 2012). In that study, the needs of the family came before those of the women and acculturative stress and socioeconomic factors interfered with their ability to make time for physical activity. Acculturation was also related to behaviors related to risk of T2D (O'Brien et al., 2014). It is possible that the women in the current study have been in the U.S. longer or did not experience these stressors to the same extent. It should also be noted that translation ILI for the prevention of T2D have historically been over-represented by women. These ILIs also report poor outcomes in 
relation to physical activity (Seidel et al., 2008; Ockene et al., 2012; Ruggiero et al., 2011).

An unawareness of healthy food options was a barrier to sustaining dietary behaviors in this study. This was described particularly in relation to sustaining dietary behavior changes. Participants indicated that at the end of the LP, they still were unsure about healthy food options, such as vegetables and how to cook them. This is consistent with barriers to healthy eating identified in high risk Hispanic populations (Rosal et al., 2011; Ramirez et al., 2007). In contrast to other focus group studies, participants did not owe this to inaccessibility of foods. Barriers identified in the literature related to healthy eating in high risk Hispanic populations included taste, access and cost (Horowitz et al., 2011).

Persistence was identified as theme in this study. Participants described setbacks or challenges which they were able to overcome by beginning again. This was consistent with a study conducted by Russell et al. (2013) with an underserved population who attended a healthy lifestyle program These authors attributed persistence to intrinsic motivation and Ryan and Deci’s (2010) Self-determination Theory. Participants in the current study used words such as motivation and self-will. Rosal et al. (2008) found that high risk Hispanic participants were more likely to refer to a lack of motivation or willpower as a barrier to dietary and physical activity, but also related that the same concepts could facilitate behavior change. In this study, motivation and will-power were identified primarily as facilitators. Participants felt that behavior changes were up to them and credited personal motivation or self-will for the changes. The concept of persistence was not identified with high risk Hispanic 
populations. It should be noted however that increasing self-efficacy was a goal in translational ILI although it was rarely measured (Horowitz et al., 2011; Kanaya et al., 2012). Self-efficacy is not just the belief in ability to perform the behavior; it is also the ability to persevere which may also theoretically explain what was identified as persistence in this study.

\section{Research question four: What was the impact of the lifestyle intervention program on the lives of participants and individual behavior changes?}

Participants identified several ways in which the lifestyle intervention program they attended had an impact on their lives. The program increased their overall awareness which was described as consciousness. Once they had the information, they described not being able to ignore what they learned. This could potentially have an impact on being able to maintain newly acquired behaviors. Participants described liking the way they were after the program and not wanting to go back. This was not identified in the diabetes prevention literature but may be related to factors presented previously. Participants may have experienced a feeling of having control over their health behavior that was not previously experienced.

Individual behavior changes had an impact on the family members and friends of those who participated in the program. This is consistent with the literature which supports inclusion of family members and family oriented approaches (Ockene et al., 2012). The literature also supports the role of matriarch in the Hispanic population and its influence on the family (D'Alonzo, 2012). In this study, male participants were more likely to relate that new physical activity behaviors had an impact on their friends supporting findings in the literature (Marquez \& McCauley, 2006). 


\section{Discussion of additional findings}

A minimum dose and duration was considered essential for effective ILI for the prevention of T2D (Ali et al., 2012; Venditti \& Kramer, 2012).A minimum of 12 sessions was recommended followed by a maintenance period of up to one year of contact. Translational ILI with high risk Hispanic populations rarely followed these recommendations. The LP in this study consisted of 8 sessions with a three month follow-up without contact. This was shorter in dose/duration than ILI with high risk Hispanic populations. It was not within the scope of this research to examine LP outcomes in relation to dose and duration. It is important to note however that many participants felt the program was insufficient in length which supports the literature. Participant requests for more sessions or prolonged contact was not noted in the literature and may reflect several factors related to this study. Participants may still doubt their ability to continue on their own. Ongoing support was an important predictor of success at one year (Knowler et al., 2002). Participants voiced their desire to co-exist which speaks to the support they received from being part of a group. Peer support has also been identified as an important factor in the literature, particularly with Hispanic populations (Ockene et al., 2012). Participants in this study may have

found peer support especially helpful, especially in those participants who reported living alone.

Low health literacy has been linked to poor health outcomes and partially explains racial disparities in health outcomes (Berkman, 2011). Although there was considerable variation in the curricula used with Hispanic populations, a common adaptation of translational ILI was to make educational materials appropriate for low 
literacy proficiency (Ockene et al., 2012; Kanaya et al., 2012; Ruggiero et al., 2011; Horowitz et al., 2011). This was the case in this study. The use of materials designed to improve health literacy was described positively in this study. There was however a notable range of participant preferences for receiving the information in the future. These included requests for more written materials to read and for note taking. Participants wanted less repetition to accommodate for additional content perhaps revealing the capacity to synthesize and take in additional information. This is in contrast to studies conducted with a Hispanic population with T2D who reported that reviewing the material at each session was beneficial (Feathers et al., 2007). The implication from this finding is that low health literacy may not be an accurate indicator of the extent individuals can take in information.

Persuasion as a recruitment strategy was supported in this study and is consistent with the literature. As previously discussed, in-person verbal persuasion either by a trusted community member, family member or friend was a noted recruitment strategy in the literature (Santoyo-Olsson et al., 2011; Parikh et al., 2010; Horowitz et al.,2009).

A relevant finding in this study was the desire to share what they learned or give testimony. Similar but somewhat less powerful findings in the literature included reports of participating in ILI to help the community (Parikh et al., 2010). One factor that may have prompted this desire is related to wanting to celebrate success as was noted previously when participants missed having an end of LP celebration. Storytelling as an intervention has been used in diabetes self-management education 
programs (Williams et al., 2014) and may be a fruitful avenue to pursue with the population under study.

\section{Theoretical Implications}

In the past decade, significant progress has been made in expanding diabetes prevention within the U.S. This expansion, however, has not been universally effective or accessible. Despite translational efforts, a lack of clarity remains as to which ILI components have the most impact on clinical outcomes in high-risk populations historically absent from the research. Theoretically, studies have been heavily influenced by a behavioral perspective and components of both the evidence-based and translational ILI have reflected this perspective. A concern of this researcher is that the persistent focus on behavior detracts from considering other factors whether at the micro or macro level.

This study found that a high risk Hispanic population attributed their ability to adopt lifestyle changes to individual, interpersonal and programmatic factors. At the individual and interpersonal level findings were consistent with SCT, particularly the construct of self-efficacy. Participant accounts were consistent with increasing levels of confidence or self-efficacy as the result of receiving support and encouragement, mastering behaviors and perseverance in performing new behaviors. Programmatic factors included role modeling of CHWs and peer support, also consistent with SCT.

This research was theoretically influenced by the social ecological and participatory perspectives with the rationale that: (1) individuals are more than their behavior; environmental factors have an influence on lifestyle behaviors and, (2) individuals and communities are experts in matters that relate to their own health. An 
ecological perspective proposes multiple influences on health behavior that range from the intrapersonal, interpersonal, community and public policy (McLeroy et al., 1988). This study identified two primary levels of influence on individual behavior which included intrapersonal factors such as knowledge and perception, and interpersonal factors such as support from family and friends. Community-based interventions are proposed to be successful only if they target all levels of influence. The LP under study remains situated within the intrapersonal and interpersonal levels of intervening as were the majority of translational ILI in the literature.

The findings of this study support further studies using a participative approach for several reasons. Participants were vocal about how they would create a LP. Recommendations, such as requesting more sessions and more physical activity were not entirely consistent with the literature. This suggests that the high risk Hispanic population in this study would benefit from being partners in intervention development. There was a desire for other members of the community to have access to the LP and to spread the word to the community which may further suggest willingness for community involvement.

An important finding of this study was that participants described the program in terms of its novelty. There was a lack of awareness regarding the information that was presented to them. This was described as the information simply not getting to them. This could be considered from a critical social theoretical perspective which proposes that historical and structural processes may be preventing them from receiving the information. As the largest segment of the U.S. health care system, nursing will increasingly be called upon to take on new roles in both patient-care and leadership 
positions. This will require novel approaches to disease prevention using different theoretical perspectives. Martins and Burbank (2011) believe that nurses will not be able to fill these roles unless they intervene at both the upstream and downstream levels. Upstream refers to structural and societal influences of health and downstream refers to individual level which can be effective but do not solve the larger problem (Martins \&Burbank, 2011). Their theory of Critical Interactionism (CI) combines symbolic interactionism and critical social theory, historically considered diverging perspectives. An example of adopting a CI theoretical perspective in relation to diabetes prevention would be developing lifestyle interventions for high risk populations while also considering the structural factors such as neighborhoods without supermarkets, or lack of access to disease prevention programs.CI offers another angle in which to view the diabetes disparity.

\section{Limitations}

There are several limitations of this study. The recruitment method used could have introduced bias. Initial plans for recruitment such as the use of flyers and then telephone calls were not fruitful. Recruitment was then conducted by inviting participants during the course of the LP they attended. Focus groups two and three took place immediately following the last session of the LP. Participants could have felt obligated to take part in the interviews although the researcher stressed the voluntary nature of the interviews. Focus groups were comprised of participants who were familiar with one another after having attended the program together. There are strengths and weaknesses to this. There was likely a degree of comfort and familiarity which decreased inhibitions and increased interaction. It is common in groups for 
members to develop roles. This can lead to members speaking for one another or implicit designation of a spokesperson who dominates the discussion (Krueger \& Casey, 2000; Morgan, 1988). These roles may have had time to develop while attending the LP. Each focus group included at least one married couple which could have potentially had an impact on what was shared by spouses. In addition, it is possible that members of groups felt the need to conform or relate experiences in a socially desirable manner. These issues were dealt with by attempting to draw in all members of the group in discussion and probe for differences as well similarities. This however does not completely eliminate these potential forms of bias. It is possible that each finding does not represent the perceptions and experiences of each participant. Refraining from answering a question can also speak volumes. Although every attempt was made to illicit responses from all participants, it is possible that some participants did not participate in answering every question fully.

This was a descriptive exploratory design with a homogenous purposive sample. Sample size was small however focus group interviews were conducted until responses became redundant, or reached saturation. Although generalizations cannot be made, rich description of the sample and setting was provided so as to allow the reader to make decisions for themselves whether these findings would transfer to other populations or settings. There was the potential for research participants to want to please the bi-lingual moderator with whom they were familiar. It was noted that participants were initially hesitant to be critical of the program yet by the end of interviews, they were more comfortable providing constructive feedback and indicating what was helpful and what was not. In addition, those who consented to be 
in this study may have been eager to share their experiences which could have influenced their responses. Although participants reported making multiple changes these were self-reported findings which may not reflect actual performance of the behavior.

The language barrier was a limitation. Although every attempt was made to reconcile translation and contextual nuances of what was said, it is possible that participant's words held a different meaning to them than what was understood by the researcher. This student researcher asked for clarification from the bi-lingual moderator and research assistants as needed during interviews who in turn would ask for clarification from participants. These attempts however began to interfere with the flow of conversation between participants and the moderator. In those situations, lively discussion and accurate detail were chosen over the understanding of the researcher in that moment. Debriefing that followed between moderator, research assistants and the researcher provided an opportunity for further clarification. Participants represented several Latin American countries and territories. Translation and transcription was conducted by a native Colombian Spanish-speaker. Though the transcriptionist was experienced in communicating with natives of each of these countries, it is possible that some words or phrases were misinterpreted.

It should be noted that, although moderation by $\mathrm{CHW}$ research assistants worked well, the language barrier for the researcher was much more difficult to overcome than anticipated. As a nurse, the most fulfilling aspect this researcher's career has been communicating with patients. Participants spoke primarily in Spanish; however, as interviews progressed, participants would sometimes speak directly to the student 
researcher hesitantly in English. This was done in a self-conscious manner, which the student researcher recognized from her own inadequacy in attempting to speak Spanish. In this respect, common ground was shared. An unanticipated benefit of not speaking the language was that this researcher was able to pay very close attention to non-verbal content such as facial expressions between participants and the degree participants appeared to deliberate over questions asked. This would not have been possible had the researcher been focused on the next question or a specific probe. The language barrier and reliance on research assistants also required a relinquishment of control. This researcher needed to rely significantly on research assistants and clinic staff which was extremely challenging. Initially uncomfortable, this reliance was subsequently valued for fostering both the respect and personal humility so vital in conducting research with populations affected by health disparities (Wallerstein \& Duran, 2006).

Research questions two and three, which related to participants being able to sustain dietary and physical activity behaviors following the completion of the LP, were not fully answered. This was due to challenges in recruiting participants of past programs. Despite increased efforts which included phone calls from not only the bilingual nursing students but also the bi-lingual CHW's, recruitment remained difficult. This led to recruiting participants during the LP which resulted in completing interviews the week immediately following the completion of the LP or at the last session of the LP. One participant, originally scheduled to be part of a focus group, was interviewed 12 weeks after completing the LP. 


\section{Implications for Nursing Research, Education and Practice}

Research. This study supports the need for further research with populations at the highest risk for developing T2D. There were multiple positive findings in this study however questions remain in relation to whether or not participants were able to sustain behavior changes and continue to persist in the face of challenges. In terms of recruitment, this study found that a high risk Hispanic population responded favorably to being persuaded or personally invited to attend a lifestyle intervention program. This has implications for nursing research with populations affected by health disparities.

Participative approaches such as CBPR are increasingly being used to conduct research with underserved populations. Several aspects of this study support using participative approaches in nursing research. The CHWs who assisted with this study were instrumental in providing feedback, building trust and understanding the experiences of the population under study. Involving CHWs in all phases of the research process could result in greater understanding. Participants of this study were vocal about how they would change the existing LP. They wanted the program to reach others in their community and felt strongly about sharing their personal stories with others. This has implications for the recruitment of future CHWs which would impact the sustainability of programs. It also provides rationale for exploring alternative components for interventions with high risk populations such as storytelling.

Education. Cultural competency has become a priority in nursing education and current recommendations include threading the concept of cultural competency 
throughout the baccalaureate nursing curriculum (Calvillo, Clarke, Ballantyne, Pacquaio, Purnell \& Villarruel, 2009). Cultural competency has been defined as the "knowledge, skills and attitudes necessary to provide care to diverse populations" (Calvillio et al, 2009, p. 2). It has been argued that teaching cultural competency reinforces the idea of othering and merely obscures the actual causes of health disparities (Drevdahl, Canales, \& Dorcy, 2008). In addition, cultural competency implies an end point versus cultural humility which is a lifelong endeavor (Tervalon \& Murrary-Garcia, 1998). The findings of this study suggest that populations need be approached without making assumptions and that culture is not a static characteristic. A major component of the translational ILI for high risk Hispanic populations was adapting for culture which included providing cultural foods, materials in Spanish and culturally relevant forms of physical activity. The individual preferences of participants in this study suggest however, that nursing education first consider individuals as just that-individuals.

Practice. This study has several implications for nursing practice and intervening with high risk populations. In their article, Using Community-Based Participatory Research to Address Health Disparities, Wallerstein \& Duran (2006) were asked by a community member, "What are you getting by working with us anyway?" upon completing a two year participatory study. This thought provoking example demonstrates how our efforts may be perceived and provides rationale for critical selfreflection in conducting research with high risk populations affected by health disparities. 
Building sustainability and seeking funding sources is another implication for nursing practice. Should the empirical findings of the LP under study prove effective, sustainability will continue to be a priority. The free clinic that offers the LP program must constantly seek funding sources to maintain programs for high risk population they serve. The nurse of the future will need to play a key role in building sustainability into lifestyle interventions aimed at reaching high risk populations in low resource settings. This includes recognizing and utilizing the expertise of community members which may be an essential in reducing disparities in health such as $\mathrm{T} 2 \mathrm{D}$ in the future.

\section{Summary}

This study explored the components of a lifestyle intervention program designed for a high risk Hispanic population and identified the facilitators and barriers to making dietary and physical activity behavior changes during and immediately following the program. The study also explored the impact of the lifestyle intervention program and lifestyle behavior change on the lives of participants with the aim of gaining an understanding from the perspectives of a high risk Hispanic population. Focus groups were viewed as a non-threatening means of data collection with the less dominant role of the researcher potentially decreasing the perception of a power differential. The emphasis on participants in focus group interviews may also empower disenfranchised populations allowing them to freely engage in discussion and reveal factors not previously considered (Halcomb et al., 2007; Krueger, 1994). The research questions were as follows: 
1. How do participants describe in their own words, the lifestyle intervention program they attended?

2. What, how and to what extent were participants able to change physical activity and dietary behaviors during the eight week program and sustain those behaviors after the program?

3. What were the facilitators and barriers described by participants in changing physical activity and dietary behaviors and the ability to maintain those behaviors after the program?

4. What was the impact of the lifestyle intervention program and individual behavior changes on the lives of participants?

This study found that a lifestyle intervention program designed for a high risk Hispanic population possessed some but not all of the components considered essential in ILI for the prevention of T2D. The LP utilized a designated curriculum, focused on dietary and physical activity and provided in-person support. These were described as important components that facilitated change in participants of this study. The program did not align with the evidence-based ILI in that it did not have specific weight loss and physical activity goals and was of significantly shorter dose and duration. Despite these omissions, the majority of participants reported adopting dietary and physical activity behaviors and subsequent weight loss. Although it was not within the scope of this study to determine the percentage of weight lost, it remains of importance in this population at significant risk for T2D.

These findings are consistent with translational ILI conducted in community settings with high risk Hispanic populations. Dose and duration was the most frequent 
modification made in those studies and similar to the LP in this study, was necessary in terms of resources and cost. The remaining issue this study was unable to resolve was that of determining how well participants were able to maintain behaviors, a noted limitation of translational ILI with high risk populations and an essential aspect of ILI. In this respect, interventions lack the intensity recommended. Intensity however has a direct impact on sustainability (Kanaya et al., 2012, Horowitz et al., 2011).

In conclusion, the LP in this study was described as a novel learning experience that provided practical information using and concise information in a supportive environment. The program was a catalyst for change. Participants voiced the desire to both maintain changes and have access to more program sessions with opportunities for physical activity. Future research is needed to further pursue these findings, explore tailoring of interventions and building sustainability for programs aimed at reducing T2D in high risk Hispanic populations. 


\section{Appendix A}

DATE: $\quad 09 / 03 / 2013$

T0: $\quad$ University of Rhode Island Institutional Review Board

FROM: $\quad$ Annie De Groot MD, Director, Medical Services, ClínicaEsperanza-Hope Clinic (CEHC)

RE: $\quad$ IRB Reference \# HU 1314-018

To the University of Rhode Island Institutional Review Board,

I am writing to affirm my support in the research proposed by Dr. Alison Tovar entitled, "Understanding the barriers and facilitators of lifestyle intervention programs for preventing diabetes in high risk Hispanic adults (496945-1)." This important project will add to our knowledge regarding the needs and preferences of our uninsured Hispanic population of RI who are disproportionately affected by diabetes. Evidence is mounting regarding the beneficial effects lifestyle intervention programs can have on the delay or progression of type 2 diabetes. Significantly less, however, is known about which designs are effective in the uninsured population. I believe that exploring program components perceived as necessary to our population will enhance the current design of the Vida Sana intervention and impact future programs at CEHC as well.

I look forward to learning about the findings of Dr. Tovar's much needed research.

Sincerely,

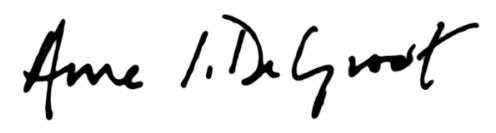

Anne De Groot, MD 
Appendix B

Focus Group Staff Screening Tool

(Please complete for each person that inquires about attending a lifestyle program focus group)

First Name:

Age:

(circle) Male Female

Ethnicity:

Date of program: Number of sessions attended?

Original reason for referral to the

program

Does the patient have diabetes? Yes

No

Preferred language to read or speak (circle) Spanish English

Plans to move out of state within next 6 months?

$$
\text { Yes_ No }
$$

Is patient willing to talk about their experience in the lifestyle program in a group setting?

$$
\text { Yes }
$$

Primary phone\# Secondary phone\#

Is there an email

address?

Are text messages okay?

Yes_ No

Is patient eligible?

Yes

No

Notes:

\section{Appendix C}

Focus Group Moderator Guide and Focus Group Questions 
Overall Structure of Focus Group

I. Welcome with explanation \& completion of paperwork (20 minutes)

II. Ground rules, confidentiality \& audiotapes

(10 minutes)

III. Dialogue and focus group questions

IV. Summary and clarification of comments

Total time allotted

(30 minutes)

2 hours (above times are estimates)

I. Welcome

a. Purpose of the focus group

i. To have participants describe their experiences while attending the lifestyle program

ii. To inform future lifestyle programs

b. Informed Consent

i. Participation is completely voluntary

ii. Participants are free to withdraw or leave at any time

c. Demographic questionnaire

i. Strictly confidential

ii. First names only

[Research personnel will roam the room when paperwork is being completed and will assist any individuals having difficulty due to language or literacy barriers.]

II. Ground Rules, Confidentiality and Audiotapes

a. Respect all opinions

b. Contributions are voluntary.

c. Confidentiality. Respect the private nature of what is heard and do not discuss outside the meeting in any way that might identify the people here.

d. Talking one at a time. Please try not to talk over each other.

e. Audiotapes are kept private and safe.

f. When the tapes are transcribed, participants will be identified by a code.

g. Anonymous quotations may go into reports or publications.

III. Dialogue and Focus Group Questions

Thank you for participating in this focus group. We appreciate your willingness to take time to participate. A focus group is a group discussion. We want you to know that each of your opinions and perspectives are important to us. There is no right or wrong answers. We only ask that you be as open and honest with us as possible. You have been chosen to participate in this focus group because you have participated in the lifestyle program.

My role is to be your guide by asking questions and keeping us on time; but this is really your time to talk. You will notice that we are taping this group in order to accurately report all ideas. Your name will not be associated with anything you say.

Are there any questions before we get started?

We are going to start with some introductions. Please tell us your first name and describe one tradition in your culture that is special to you.

Now that we are getting to know each other, let's go on to the questions we have for you today. 
1. We'd like to start by talking about your experiences with the program. Can you please describe the program that you attended? Was there anything not mentioned? Did anyone have something different happen in their program?

2. What changes were you were able to make while you were in the program? Let's make a list. (We will use a LARGE PIECE OF PAPER OR EASEL for this). What kind of changes in your diet? What kind of changes in exercise? How did you make these changes? Looking at the list we made, which of the changes did you find hard? What about easy? (Use red and green markers to identify)

3. Can you describe any strategies or tips that you learned that helped you make changes in your diet or activity level? What about strategies that you came up with on your own?

4. Was there someone or something in particular that helped you with making changes? How did they help you? Is there anyone who helped you that we didn't mention?

5. Is there anything that got in the way of making changes during the program? Thinking back, can you remember a particularly stressful or bad day that you had? Can you describe that day? What did you do? What about something that prevented you after the program?

6. We'd like to know how you are doing since the program ended. Can you tell us about what your life is like now compared to before the program?

7. Some people have asked for the program to continue. If you could create the program, what would they be like? What would you suggest? How would you like to receive the information? Where would you choose to have the program?

8. If you did not attend all 8 of the program sessions, what were some of the reasons why?

IV. Summary and Clarification of Comments

The moderator will ask if there is anything that was not mentioned that participants would like to add. The moderator will summarize the interview with participants, the assistant moderator and the research assistant taking field notes to provide an opportunity to further clarify any comments. Participants will be asked if they concur with the summary. Participants will be thanked for their participation. 


\section{Appendix D}

\section{CONSENT FORM FOR RESEARCH}

You have been invited to participate in the University of Rhode Island research project described below. The researcher will explain the project to you in detail. You should feel free to ask questions. If you have additional questions regarding the study, please contact the principal investigator, Dr. Alison Tovar, by email at alison_tovar@mail.uri.edu or by phone at 401-874-9855 or Lisa DiMaria, the student investigator at ladimaria@mail.uri.edu. To participate you must have attended the Vida Sana program, be low income and over the age of 18.

\section{Description of the project}

This research project explores the descriptions and experiences of people who have attended the healthy living lifestyle program and the factors that were helpful or were difficult for participants in making dietary and activity changes related to weight loss. The project will also explore the different parts of the Vida Sana program and make comparisons to other well known programs. The results will help inform future diabetes prevention programs.

\section{What will be done}

If you choose to participate, you will be asked to take part in a discussion group, called a focus group, with other participants. You have already provided information via the telephone or in person including: your age, gender, language preference and ethnicity. We used this information to schedule you for the discussion group. Your total involvement should be no longer than 2 hours.

For the discussion, all persons will be identified by first names only. These groups will be digitally recorded and recordings will be transcribed. For the discussion you will be asked to describe your experiences while attending the healthy living program and share your ideas about what was most helpful and what made change difficult. There is no right or wrong answers; we just want to hear everyone's descriptions and thoughts.

\section{Risks}

The risk to participate is minimal. We ask that group members do not share names or what is said in the focus group, but cannot guarantee complete confidentiality. We will identify you by your first name only. All names will be removed before transcripts are analyzed.

\section{Benefits of the study}

Though you may not benefit directly from this study, the results will provide valuable information on how to design effective diabetes prevention programs for Hispanics. This will help inform future programs and interventions. You will receive a supermarket gift card to thank you for being in the study.

\section{Confidentiality}

All information that you provide will be kept confidential and your privacy will be protected to the maximum extent allowable by law. For the discussion group, you will only be identified by your first name. All identifying information, including names, will be deleted from the transcript before data analysis. Transcripts as well as the original tapes will be stored in locked offices at The University of Rhode Island for up to five years (as required by law) and 
then destroyed. Data will be reported in summary format, and no names will be used. Selected quotes from the discussion groups may be reported but without names.

\section{Decision to quit at any time}

Your participation is voluntary. You may refuse to answer any questions that are part of the discussion group. You may stop participating at any point. Whatever you decide will in no way affect the care you receive at Clinica Esperanza. If you wish to quit, simply inform the Program Manager, Ingrid Castillo or Rosa Roman, Navegante, at Clinica Esperanza of your decision at 401-347-9093. Please also inform Alison Tovar by email at alison_tovar@mail.uri.edu.

\section{Questions}

If you have any questions or concerns, please contact the principal investigator, Dr. Alison Tovar, by email at alison_tovar@mail.uri.edu or by phone at 401-874-9855. If you have concerns regarding your rights as a research participant, please contact the Office of the Vice President for Research and Economic Development at 401-874-4328. This study has been approved by the Institutional Review Board (IRB) of the University of Rhode Island.

Thank you for your time and interest in this study.

I have read the consent form to participate in this research study, and my questions have been answered. By signing below, I agree to take part in the discussion group. All information will remain confidential. I understand that I can choose not to participate at any time. Please sign both consent forms, keeping one for you.

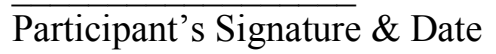

$\overline{\text { Participant's Printed Name }}$

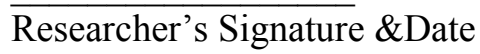

$\overline{\text { Researcher's Printed Name }}$

I understand that the discussion group will be tape recorded and tapes will be transcribed, but names and identifying information will be removed from the transcripts before data analysis.

Participant's Signature \& Date

Participant's Printed Name
Researcher's Signature \& Date

Researcher's Printed Name 
Appendix E

Vida Sana Focus Group Demographic Questionnaire

First Name:

Study ID\#

Age:

Country born in (circle one):

Colombia

Dominican Republic

El Salvador

Guatemala

Mexico

Puerto Rico

United States

Other (please provide):

If you were born in the U.S., which country were your parents born in (circle one)?

Colombia

Dominican Republic

El Salvador

Guatemala

Mexico

Puerto Rico

United States

Other (please provide):

What language do you prefer to read and speak? (check one)

Spanish

English

Other

How many adults live with you? (circle one)

1

2

3

4 or more 
How many children live with you? (circle one)

1

2

3

4 or more

Employment status:

Unemployed

Part time employment

Full time employment

Please check what best describes your educational experience:

Less than high school

High school diploma or equivalent

Some college

College degree

Month attended Vida Sana Program:

Location of Vida Sana Program:

Clinica Esperanza

Open Table of Christ

Gloria Dei

Nickerson House

Of the 8 Vida Sana sessions, how many did you attend (circle one)?

$\begin{array}{llllllll}1 & 2 & 3 & 4 & 5 & 6 & 7 & 8\end{array}$

If it has been 3 months since you attended the Vida Sana program, were you able to attend the 3 month follow up?

Yes

No

Did you attend more than one 8 week Vida Sana program?

Yes

No 


\section{BIBLIOGRAPHY}

Abraído-Lanza, A. F., Armbrister, A. N., Flórez, K. R., \& Aguirre, A. N. (2006).

Toward a theory-driven model of acculturation in public health research. American Journal of Public Health, 96(8), 1342-1346.

Ackermann, R. T., Finch, E. A., Brizendine, E., Zhou, H., \& Marrero, D. G. (2008).

Translating the diabetes prevention program into the community: The DEPLOY pilot study. American Journal of Preventive Medicine, 35(4), 357-363. doi:

10.1016/j.amepre.2008.06.035

Ackermann, R. T., Finch, E. A., Caffrey, H. M., Lipscomb, E. R., Hays, L. M., \& Saha, C. (2011). Long-term effects of a community-based lifestyle intervention to prevent type 2 diabetes: The DEPLOY extension pilot study. Chronic Illness, 7(4), 279-290. doi:10.1177/1742395311407532

Agardh, E., Allebeck, P., Hallqvist, J., Moradi, T., \& Sidorchuk, A. (2011). Type 2 diabetes incidence and socio-economic position: A systematic review and metaanalysis. International Journal of Epidemiology, 40(3), 804-

818.doi:10.1093/ije/dyr029

Ai, A. L., Appel, H. B., Huang, B., \& Lee, K. (2012). Overall health and healthcare utilization among Latino American women in the United States. Journal of Women's Health, 21(8), 878-885.

Ali, M. K., Bullard, K. M., Saaddine, J. B., Cowie, C. C., Imperatore, G., \& Gregg, E. W. (2013). Achievement of goals in US diabetes care, 1999-2010. New England Journal of Medicine, 368(17), 1613-1624.

Ali, M. K., Echouffo-Tcheugui, J., \& Williamson, D. F. (2012). How effective were 
lifestyle interventions in real-world settings that were modeled on the diabetes prevention program? Health Affairs (Project Hope), 31(1), 67-75. doi:10.1377/hlthaff.2011.1009

American Diabetes Association. (2011). Diabetes statistics. Retrieved from http://www.diabetes.org/diabetes-basics/diabetes-statistics/?loc=DropDownDBstats

American Diabetes Association. (2013). Economic costs of diabetes in the U.S. in 2012. Diabetes Care, 36(4), 1033-1046. doi:10.2337/dc12-2625

Amundson, H. A., Butcher, M. K., Gohdes, D., Hall, T. O., Harwell, T. S., Helgerson, S. D., ... Montana Cardiovascular Disease and Diabetes Prevention Program Workgroup. (2009). Translating the diabetes prevention program into practice in the general community: Findings from the Montana cardiovascular disease and diabetes prevention program. The Diabetes Educator, 35(2), 209-10, 213-4, 21620 passim. doi:10.1177/0145721709333269

Bandura, A. (1977). Self-efficacy: Toward a unifying theory of behavioral change. Psychological Review, 84(2), 191.

Bandura, A. (1986). Social foundations of thought and action: A social cognitive theory. Upper Saddle River, NJ: Prentice-Hall.

Bandura, A. (1991). Social cognitive theory of self-regulation. Organizational Behavior and Human Decision Processes, 50(2), 248-287.

Bandura, A. (2002). Social cognitive theory in cultural context. Applied Psychology, 51(2), 269-290.

Bandura, A. (2004). Health promotion by social cognitive means. Health Education \& 
Behavior : The Official Publication of the Society for Public Health Education, 31(2), 143-164. doi:10.1177/1090198104263660

Bandura, A. (2006). Toward a psychology of human agency. Perspectives on Psychological Science, 1(2), 164-180.

Baranowski, T., Perry, C. L., \& Parcel, G. S. (2002). How individuals, environments, and health behavior interact. In K. Glanz, B.K. Rimer, \& F.M. Lewis (Eds.), Health Behavior and Health Education: Theory, Research, and Practice (3rd ed.; pp. 165-184).San Francisco: Jossey-Bass.

Barrera, M., Jr, Castro, F. G., Strycker, L. A., \& Toobert, D. J. (2013). Cultural adaptations of behavioral health interventions: A progress report. Journal of Consulting and Clinical Psychology, 81(2), 196-205. doi:10.1037/a0027085

Beck, A. T. (1964). Thinking and depression: II. Theory and therapy. Archives of General Psychiatry, 10(6), 561-571.

Berkman, N. D., Sheridan, S. L., Donahue, K. E., Halpern, D. J., \& Crotty, K. (2011). Low health literacy and health outcomes: An updated systematic review. Annals of Internal Medicine, 155(2), 97-107.

Bernal, G., Jiménez-Chafey, M. I., \& Domenech Rodríguez, M. M. (2009). Cultural adaptation of treatments: A resource for considering culture in evidence-based practice. Professional Psychology: Research and Practice, 40(4), 361.

Blackwell, D. L., Lucas, J. W., \& Clarke, T. C. (2014). Summary health statistics for U.S. adults: National health interview survey, 2012. Vital and Health Statistics. Series 10(260), 1-161.

Boltri, J. M., Davis-Smith, M., Okosun, I. S., Seale, J. P., \& Foster, B. (2011). 
Translation of the national institutes of health diabetes prevention program in African American churches. Journal of the National Medical Association, 103(3), 194-202.

Boltri, J. M., Davis-Smith, Y. M., Seale, J. P., Shellenberger, S., Okosun, I. S., \& Cornelius, M. E. (2008). Diabetes prevention in a faith-based setting: Results of translational research. Journal of Public Health Management and Practice, 14(1), 29-32. doi:10.1097/01.PHH.0000303410.66485.91

Boltri, J. M., Davis-Smith, Y. M., Zayas, L. E., Shellenberger, S., Seale, J. P., Blalock, T. W., \& Mbadinuju, A. (2006). Developing a church-based diabetes prevention program with African Americans: Focus group findings. The Diabetes Educator, 32(6), 901-909. doi:32/6/901

Bradbury, H., \& Reason, P. (2003). Issues and choice points for improving the quality of action research. In Minkler, M. \& N. Wallerstein (Eds.), Community Based Participatory Research for Health. (pp. 201-220). San Francisco: Jossey-Bass.

Bridle, C., Riemsma, R. P., Pattenden, J., Sowden, A. J., Mather, L., Watt, I. S., \& Walker, A. (2005). Systematic review of the effectiveness of health behavior interventions based on the transtheoretical model. Psychology \& Health, 20(3), 283-301.

Brown, C. E., Wickline, M. A., Ecoff, L., \& Glaser, D. (2009). Nursing practice, knowledge, attitudes and perceived barriers to evidence-based practice at an academic medical center. Journal of Advanced Nursing, 65(2), 371-381.

Bruehl, H., Sweat, V., Hassenstab, J., Polyakov, V., \& Convit, A. (2010). Cognitive impairment in nondiabetic middle-aged and older adults is associated with insulin 
resistance. Journal of Clinical \& Experimental Neuropsychology, 32(5), 487-493. doi:10.1080/13803390903224928

Bruehl, H., Wolf, O. T., Sweat, V., Tirsi, A., Richardson, S., \& Convit, A. (2009). Modifiers of cognitive function and brain structure in middle-aged and elderly individuals with type 2 diabetes mellitus. Brain Research, 1280, 186-194.

Buckley, J., Yekta, S., Joseph, V., Johnson, H., Oliverio, S., \& De Groot, A. S. (2015). Vida Sana: A lifestyle intervention for uninsured, predominantly Spanish-speaking immigrants improves metabolic syndrome indicators. Journal of Community Health, 40, 116-123. doi:DOI 10.1007/s10900-014-9905-z

Burke, L. E., Wang, J., \& Sevick, M. A. (2011). Self-monitoring in weight loss: A systematic review of the literature. Journal of the American Dietetic Association, 111(1), 92-102.

Butryn, M. L., Phelan, S., Hill, J. O., \& Wing, R. R. (2007). Consistent selfmonitoring of weight: A key component of successful weight loss maintenance. Obesity, 15(12), 3091-3096.

Caballero, A. E. (2005). Diabetes in the Hispanic or Latino population: Genes, environment, culture, and more. Current Diabetes Reports, 5(3), 217-225.

Calvillo, E., Clark, L., Ballantyne, J. E., Pacquiao, D., Purnell, L. D., \& Villarruel, A. M. (2009). Cultural competency in baccalaureate nursing education. Journal of Transcultural Nursing : Official Journal of the Transcultural Nursing Society / Transcultural Nursing Society, 20(2), 137-145. doi:10.1177/1043659608330354

Castro, F. G., Barrera, M., Jr., \& Holleran Steiker, L. K. (2010). Issues and challenges in the design of culturally adapted evidence-based interventions. Annual Review of 
Clinical Psychology, 6, 213-239.

Centers for Disease Control and Prevention. (2011). Health disparities and inequalities report--United States. ( No. 60).

Centers for Disease Control and Prevention. (May 15, 2014). National diabetes prevention program. Retrieved from http://www.cdc.gov/diabetes/prevention/index.htm

Centers for Disease Control and Prevention. (January 1, 2015). Diabetes prevention recognition program. Retrieved from http//www.cdc.gov/diabetes/prevention/recognition/standards/htm.

Chaufan, C., \& Weitz, R. (2009). The elephant in the room: The invisibility of poverty in research on type 2 diabetes. Humanity \& Society, 33, 74-98.

Clemans-Cope, L., Kenney, G. M., Buettgens, M., Carroll, C., \& Blavin, F. (2012). The Affordable Care Act's coverage expansions will reduce differences in uninsurance rates by race and ethnicity. Health Affairs, 31(5), 920-930. doi:10.1377/hlthaff.2011.1086

Cohen, D. J., Crabtree, B. F., Etz, R. S., Balasubramanian, B. A., Donahue, K. E., Leviton, L. C., . . Green, L. W. (2008). Fidelity versus flexibility: Translating evidence-based research into practice. American Journal of Preventive Medicine, 35(5), S381-S389.

Cohen, R. A., \& Martinez, M. E. (2012). Health insurance coverage: Early release of estimates from the national health interview survey, January-March 2012. Atlanta, GA: Centers for Disease Control and Prevention.

Community Health Innovations of Rhode Island. (2015) http://chi-ri.org. Accessed 
October 25, 2015.

Craft, S. (2007). Insulin resistance and Alzheimer's disease pathogenesis: Potential mechanisms and implications for treatment. Current Alzheimer Research, 4(2), $147-152$.

Crane, P. K., Walker, R., Hubbard, R. A., Li, G., Nathan, D. M., Zheng, H., . . Larson, E. B. (2013). Glucose levels and risk of dementia. The New England Journal of Medicine, 369(6), 540-548. doi:10.1056/NEJMoa1215740

Dall, T. M., Zhang, Y., Chen, Y. J., Quick, W. W., Yang, W. G., \& Fogli, J. (2010). The economic burden of diabetes. Health Affairs, 29(2), 297-303. doi:10.1377/hlthaff.2009.0155

Dallman, M. F. (2010). Stress-induced obesity and the emotional nervous system. Trends in Endocrinology \& Metabolism, 21(3), 159-165.

D'Alonzo, K. T. (2012). The influence of marianismo beliefs on physical activity of immigrant Latinas. Journal of Transcultural Nursing : Official Journal of the Transcultural Nursing Society / Transcultural Nursing Society, 23(2), 124-133. doi: $10.1177 / 1043659611433872$

Davis-Smith, Y. M., Boltri, J. M., Seale, J. P., Shellenberger, S., Blalock, T., \& Tobin, B. (2007). Implementing a diabetes prevention program in a rural AfricanAmerican church. Journal of the National Medical Association, 99(4), 440-446.

Delgadillo, A. T., Grossman, M., Santoyo-Olsson, J., Gallegos-Jackson, E., Kanaya, A. M., \& Stewart, A. L. (2010). Description of an academic community partnership lifestyle program for lower income minority adults at risk for diabetes. The Diabetes Educator, 36(4), 640-650. doi:10.1177/0145721710374368 
Diabetes Prevention Program (DPP) Research Group. (2002). The diabetes prevention program (DPP): Description of lifestyle intervention. Diabetes Care, 25(12), 21652171.

Diabetes Prevention Program Research Group. (2003). Within-trial cost-effectiveness of lifestyle intervention or metformin for the primary prevention of type 2 diabetes. Diabetes Care, 26(9), 2518-2523.

Diabetes Prevention Program Research Group, Knowler, W. C., Fowler, S. E., Hamman, R. F., Christophi, C. A., Hoffman, H. J., . . Nathan, D. M. (2009). 10year follow-up of diabetes incidence and weight loss in the diabetes prevention program outcomes study. Lancet, 374(9702), 1677-1686. doi:10.1016/S01406736(09)61457-4

Dickens, L., \& Watkins, K. (1999). Action research: Rethinking Lewin. Management Learning, 30(2), 127-140.

Drevdahl, D. J., Canales, M. K., \& Dorcy, K. S. (2008). Of goldfish tanks and moonlight tricks: Can cultural competency ameliorate health disparities? ANS.Advances in Nursing Science, 31(1), 13-27. doi:10.1097/01.ANS.0000311526.27823.05

D'Zurilla, T. J., \& Goldfried, M. R. (1971). Problem solving and behavior modification. Journal of Abnormal Psychology, 78(1), 107.

Ellis, A. (1980). Rational-emotive therapy and cognitive behavior therapy: Similarities and differences. Cognitive Therapy and Research, 4(4), 325-340.

Eriksson, J., Lindström, J., Valle, T., Aunola, S., Hämäläinen, H., Ilanne-Parikka, P., . .. Tuomilehto, J. (1999). Prevention of type II diabetes in subjects with impaired 
glucose tolerance: The diabetes prevention study (DPS) in Finland. Diabetologia, 42(7), 793- 801. doi:10.1007/s001250051229

Fain, J. A. (2013). Reading, understanding, and applying nursing research (3rd ed.). Philadelphia: FA Davis.

Feathers, J. T., Kieffer, E. C., Palmisano, G., Anderson, M., Janz, N., Spencer, M. S., . . . James, S. A. (2007). The development, implementation, and process evaluation of the REACH detroit partnership's diabetes lifestyle intervention The Diabetes Educator, 33(3), 509-520. doi:10.1177/0145721707301371

Ford, E. S., Giles, W. H., \& Dietz, W. H. (2002). Prevalence of the metabolic syndrome among US adults: Findings from the third national health and nutrition examination survey. Jama, 287(3), 356-359.

Frey, J. H., \& Fontana, A. (1991). The group interview in social research. The Social Science Journal, 28(2), 175-187. doi:10.1016/0362-3319(91)90003-M

Frohlich, K. L., \& Potvin, L. (2008). Transcending the known in public health practice: The inequality paradox: The population approach and vulnerable populations. American Journal of Public Health, 98(2), 216-221. doi:10.2105/AJPH.2007.114777

Gabir, M. M., Hanson, R. L., Dabelea, D., Imperatore, G., Roumain, J., Bennett, P. H., \& Knowler, W. C. (2000). The 1997 American Diabetes Association and 1999 World Health Organization criteria for hyperglycemia in the diagnosis and prediction of diabetes. Diabetes Care, 23(8), 1108-1112. doi:10.2337/diacare.23.8.1108

Gavin, J., Alberti, K., Davidson, M., DeFronzo, R., Drash, A., Gabbe, S., . . . Keen, H. 
(1997). Report of the expert committee on the diagnosis and classification of diabetes mellitus. Diabetes Care, 20(7), 1183-1197.

Geller, S., Taylor, B. M., \& Scott, H. D. (2004). Free clinics helping to patch the safety net. Journal of Health Care for the Poor and Underserved, 15(1), 42-51.

Glasgow, R. E. (2003). Translating research to practice: Lessons learned, areas for improvement, and future directions. Diabetes Care, 26(8), 2451-2456.

Glasgow, R. E., Lichtenstein, E., \& Marcus, A. C. (2003). Why don't we see more translation of health promotion research to practice? rethinking the efficacy-toeffectiveness transition. American Journal of Public Health, 93(8), 1261-1267.

Green, L. W., \& Glasgow, R. E. (2006). Evaluating the relevance, generalization, and applicability of research issues in external validation and translation methodology. Evaluation \& the Health Professions, 29(1), 126-153.

Grundy, S. M. (2012). Pre-diabetes, metabolic syndrome, and cardiovascular risk. Journal of the American College of Cardiology, 59(7), 635-643.

Grzywacz, J. G., \& Fuqua, J. (2000). The social ecology of health: Leverage points and linkages. Behavioral Medicine, 26(3), 101. Retrieved from http://search.ebscohost.com/login.aspx?direct $=$ true $\& d b=a 9 h \& A N=4070325 \&$ site $=$ ehost-live

Halcomb, E. J., Gholizadeh, L., DiGiacomo, M., Phillips, J., \& Davidson, P. M. (2007). Literature review: Considerations in undertaking focus group research with culturally and linguistically diverse groups. Journal of Clinical Nursing, 16(6), 1000-1011. doi:10.1111/j.1365-2702.2006.01760.x

Hamman, R. F., Wing, R. R., Edelstein, S. L., Lachin, J. M., Bray, G. A., Delahanty, 
L., . . Wylie-Rosett, J. (2006). Effect of weight loss with lifestyle intervention on risk of diabetes. Diabetes Care, 29(9), 2102-2107. doi:10.2337/dc06-0560

Hernan, W. H., Brandle, M., Zhang, P., Williamson, D. F., Matulik, M. J., Ratner, R. E., . . . Diabetes Prevention Program Research Group. (2003). Costs associated with the primary prevention of type 2 diabetes mellitus in the diabetes prevention program. Diabetes Care, 26(1), 36-47.

Hettema, J., Steele, J., \& Miller, W. R. (2005). Motivational interviewing. Annual Review of Clinical Psychology, 1, 91-111.

Hispanic Community Health Study/Study of Latinos Data Book: A Report to the Communities. (2013). ( No. 13-7951). Bethesda, MD: U.S. Department of Health and Human Services.

Horowitz, C. R., Eckhardt, S., Talavera, S., Goytia, C., \& Lorig, K. (2011). Effectively translating diabetes prevention: A successful model in a historically underserved community. Translational Behavioral Medicine, 1(3), 443-452.

Horowitz, C. R., Brenner, B. L., Lachapelle, S., Amara, D. A., \& Arniella, G. (2009). Effective recruitment of minority populations through community-led strategies American Journal of Preventive Medicine, 37(6 Suppl 1), S195-200. doi:10.1016/j.amepre.2009.08.006

Horowitz, C. R., Tuzzio, L., Rojas, M., Monteith, S. A., \& Sisk, J. E. (2004). How do urban African Americans and Latinos view the influence of diet on hypertension? Journal of Health Care for the Poor and Underserved, 15(4), 631-644. doi:S1548686904406317

Huang, E. S., Basu, A., O'Grady, M., \& Capretta, J. C. (2009). Projecting the future 
diabetes population size and related costs for the U.S.. Diabetes Care, 32(12), 2225-2229. doi:10.2337/dc09-0459

Huer, M. B., \& Saenz, T. I. (2003). Challenges and strategies for conducting survey and focus group research with culturally diverse groups. American Journal of Speech-Language Pathology, 12(2), 209-220.

Institute of Medicine. (2003). Unequal treatment: Confronting racial and ethnic disparities in health care. Washington, DC: National Academies Press.

Institute of Medicine. (2009). Initial national priorities for comparative effectiveness research. Washington, DC: National Academies Press.

Institute of Medicine. (2011). The future of nursing: Leading change, advancing health. Washington, DC: National Academies Press.

Inzucchi, S. E., Bergenstal, R. M., Buse, J. B., Diamant, M., Ferrannini, E., Nauck, M., . . Matthews, D. R. (2012). Management of hyperglycemia in type 2 diabetes: A patient-centered approach: Position statement of the American Diabetes Association (ADA) and the European Association for the Study of Diabetes (EASD). Diabetes Care, 35(6), 1364-1379. doi:10.2337/dc12-0413

Israel, B., Eng, E., Schulz, A., \& Parker, E. (2005). Introduction to methods in CBPR for health. In Israel, B.A., E. Eng, A. Schulz, E.A. Parker, \& D. Satcher (Eds.), Methods in Community-Based Participatory Research for Health, (pp. 3-26). San Francisco: Jossey-Bass.

Jarrett, R., Keen, H., Fuller, J., \& McCartney, M. (1979). Worsening to diabetes in men with impaired glucose tolerance (“borderline diabetes”). Diabetologia, 16(1), 25-30. 
Kanaya, A. M., Santoyo-Olsson, J., Gregorich, S., Grossman, M., Moore, T., \& Stewart, A. L. (2012). The live well, be well study: A community-based, translational lifestyle program to lower diabetes risk factors in ethnic minority and lower-socioeconomic status adults. American Journal of Public Health, 102(8), 1551-1558. doi:10.2105/AJPH.2011.300456

Katula, J. A., Vitolins, M. Z., Rosenberger, E. L., Blackwell, C., Espeland, M. A., Lawlor, M. S., . . Goff, D. C. (2010). Healthy living partnerships to prevent diabetes (HELP PD): Design and methods. Contemporary Clinical Trials, 31(1), $71-81$.

Katula, J. A., Vitolins, M. Z., Morgan, T. M., Lawlor, M. S., Blackwell, C. S., Isom, S. P., . . Goff, D. C., Jr.. (2013). The healthy living partnerships to prevent diabetes study: 2-year outcomes of a randomized controlled trial. American Journal of Preventive Medicine, 44(4 Suppl 4), S324-32. doi:10.1016/j.amepre.2012.12.015

Katula, J. A., Vitolins, M. Z., Rosenberger, E. L., Blackwell, C. S., Morgan, T. M., Lawlor, M. S., \& Goff, D. C.,Jr. (2011). One-year results of a community-based translation of the diabetes prevention program: Healthy-living partnerships to prevent diabetes (HELP PD) project. Diabetes Care, 34(7), 1451-1457. doi:10.2337/dc10-2115

Kieffer, E. C., Willis, S. K., Odoms-Young, A. M., Guzman, J. R., Allen, A. J., Two Feathers, J., \& Loveluck, J. (2004). Reducing disparities in diabetes among African-American and Latino residents of Detroit: The essential role of community planning focus groups. Ethnicity \& Disease, 14(3 Suppl 1), S27-37. 
Kim, H. S. (2010). The nature of theoretical thinking in nursing (3rd ed.). New York, NY: Springer Publishing Company.

Kim, H. S. (2000). An integrative framework for conceptualizing clients: A proposal for a nursing perspective in the new century. Nursing Science Quarterly, 13(1), 3740. doi:10.1177/08943180022107258

Kitabchi, A. E., Temprosa, M., Knowler, W. C., Kahn, S. E., Fowler, S. E., Haffner, S. M., . . Diabetes Prevention Program Research Group. (2005). Role of insulin secretion and sensitivity in the evolution of type 2 diabetes in the diabetes prevention program: Effects of lifestyle intervention and metformin. Diabetes, 54(8), 2404-2414.

Kitzinger, J. (2008). The methodology of focus groups: The importance of interaction between research participants. Sociology of Health \& Illness, 16, 103-121.

Knowler, W. C., Barrett-Connor, E., Fowler, S. E., Hamman, R. F., Lachin, J. M., Walker, E. A., . . . Diabetes Prevention Program Research Group. (2002). Reduction in the incidence of type 2 diabetes with lifestyle intervention or metformin. The New England Journal of Medicine, 346(6), 393-403. doi:10.1056/NEJMoa012512

Kolb, A. Y., \& Kolb, D. A. (2005). Learning styles and learning spaces: Enhancing experiential learning in higher education. Academy of Management Learning \& Education, 4(2), 193-212.

Kramer, M. K., Kriska, A. M., Venditti, E. M., Miller, R. G., Brooks, M. M., Burke, L. E., .. Orchard, T. J. (2009). Translating the diabetes prevention program: A comprehensive model for prevention training and program delivery. American 
Journal of Preventive Medicine, 37(6), 505-511.

Kramer, M. K., Kriska, A. M., Venditti, E. M., Semler, L. N., Miller, R. G., McDonald, T., ... Orchard, T. J. (2010). A novel approach to diabetes prevention: Evaluation of the group lifestyle balance program delivered via DVD. Diabetes Research and Clinical Practice, 90(3), e60-3. doi:10.1016/j.diabres.2010.08.013

Kramer, M. K., McWilliams, J. R., Chen, H. Y., \& Siminerio, L. M. (2011). A community-based diabetes prevention program: Evaluation of the group lifestyle balance program delivered by diabetes educators. The Diabetes Educator, 37(5), 659-668. doi:10.1177/0145721711411930

Kreuter, M. W., Lukwago, S. N., Bucholtz, R. D., Clark, E. M., \& Sanders-Thompson, V. (2003). Achieving cultural appropriateness in health promotion programs: Targeted and tailored approaches. Health Education \& Behavior : The Official Publication of the Society for Public Health Education, 30(2), 133-146.

Krieger, N. (2001). Theories for social epidemiology in the 21st century: An ecosocial perspective. International Journal of Epidemiology, 30(4), 668-677.

Krueger, R. A. (1994). Focus groups: A practical guide for applied research (2nd ed.). Thousand Oaks, CA: Sage Publications.

Krueger, R. A. (1997). Analyzing and reporting focus group results. London: Sage publications.

Krueger, R. A., \& Casey, M. A. (2000). Focus groups: A practical guide for applied research (3rd ed.). Thousand Oaks, CA: Sage Publications.

Kumanyika, S. (2008). Ethnic minorities and weight control research priorities: Where are we now and where do we need to be? Preventive Medicine, 47(6), 583-586. 
Landreneau, K. J., \& Ward-Smith, P. (2007). Perceptions of adult patients on hemodialysis concerning choice among renal replacement therapies. Nephrology Nursing Journal, 34(5), 513.

Larsen, B. A., Noble, M. L., Murray, K. E., \& Marcus, B. H. (2014). Physical activity in Latino men and women facilitators, barriers, and interventions. American Journal of Lifestyle Medicine, 9(1), 4-30. doi: 10.1177/1559827614521758

Lawlor, M. S., Blackwell, C. S., Isom, S. P., Katula, J. A., Vitolins, M. Z., Morgan, T. M., \& Goff, D. C., Jr.. (2013). Cost of a group translation of the diabetes prevention program: Healthy living partnerships to prevent diabetes. American Journal of Preventive Medicine, 44(4 Suppl 4), S381-9. doi:10.1016/j.amepre.2012.12.016

Li, R., Zhang, P., Barker, L. E., Chowdhury, F. M., \& Zhang, X. (2010). Costeffectiveness of interventions to prevent and control diabetes mellitus: A systematic review. Diabetes Care, 33(8), 1872-1894. doi:10.2337/dc10-0843

Lincoln, Y., S., \& Guba, E., G. (1985). Naturalistic inquiry. Beverly Hills, CA: Sage.

Lincoln, Y. S., \& Guba, E. G. (1986). But is it rigorous? Trustworthiness and authenticity in naturalistic evaluation. New Directions for Program Evaluation, 1986(30), 73-84. doi:10.1002/ev.1427

Lindström, J., Louheranta, A., Mannelin, M., Rastas, M., Salminen, V., Eriksson, J., . . . Tuomilehto, J. (2003). The Finnish diabetes prevention study (DPS): Lifestyle intervention and 3-year results on diet and physical activity. Diabetes Care, 26(12), 3230-3236. doi:10.2337/diacare.26.12.3230

Link, C. L., \& McKinlay, J. B. (2009). Disparities in the prevalence of diabetes: Is it 
race/ethnicity or socioeconomic status? Results from the Boston area community health (BACH) survey. Ethnicity \& Disease, 19(3), 288-292.

Long, S. D., O’Brien, K., Macdonald, K. G., Leggett-Frazier, N., Swanson, M. S., Pories, W. J., \& Caro, J. F. (1994a). Weight loss in severely obese subjects prevents the progression of impaired glucose tolerance to type II diabetes: A longitudinal interventional study. Diabetes Care, 17(5), 372-375. doi:10.2337/diacare.17.5.372

Lorenzo, C., Okoloise, M., Williams, K., Stern, M. P., Haffner, S. M., \& San Antonio Heart Study. (2003). The metabolic syndrome as predictor of type 2 diabetes: The San Antonio heart study. Diabetes Care, 26(11), 3153-3159.

Lorenzo, C., Williams, K., Hunt, K. J., \& Haffner, S. M. (2007). The national cholesterol education program - adult treatment panel III, International Diabetes Federation, and World Health Organization definitions of the metabolic syndrome as predictors of incident cardiovascular disease and diabetes. Diabetes Care, 30(1), 8-13. doi:10.2337/dc06-1414

MacNaughton, N. S. (2008). Health disparities and health-seeking behavior among Latino men: A review of the literature. Journal of Transcultural Nursing, 19(1), 83-91. doi:10.1177/1043659607309144

Makosky Daley, C., James, A. S., Ulrey, E., Joseph, S., Talawyma, A., Choi, W. S., . . . Coe, M. K. (2010). Using focus groups in community-based participatory research: Challenges and resolutions. Qualitative Health Research, 20(5), 697706. doi:10.1177/1049732310361468

Marquez, D. X., \& McAuley, E. (2006). Gender and acculturation influences on 
physical activity in Latino adults. Annals of Behavioral Medicine, 31(2), 138-144.

Martins, D. C., \& Burbank, P. M. (2011). Critical interactionism: An upstreamdownstream approach to health care reform. ANS.Advances in Nursing Science, 34(4), 315-329. doi:10.1097/ANS.0b013e3182356c19

Mattei, J., Demissie, S., Falcon, L. M., Ordovas, J. M., \& Tucker, K. (2010). Allostatic load is associated with chronic conditions in the Boston Puerto Rican health study. Social Science \& Medicine (1982), 70(12), 1988-1996. doi:10.1016/j.socscimed.2010.02.024

Mau, M. K., Keawe'aimoku Kaholokula, J., West, M. R., Leake, A., Efird, J. T., Rose, C., ... Gomes, H. (2010). Translating diabetes prevention into native Hawaiian and Pacific Islander communities: The PILI 'ohana pilot project. Progress in Community Health Partnerships : Research, Education, and Action, 4(1), 7-16. doi:10.1353/cpr.0.0111

McLeroy, K. R., Bibeau, D., Steckler, A., \& Glanz, K. (1988). An ecological perspective on health promotion programs. Health Education \& Behavior, 15(4), 351-377.

McCloskey, J. (2009). Promotores as partners in a community-based diabetes intervention program targeting Hispanics. Family \& Community Health, 32(1), 4857. doi:10.1097/01.FCH.0000342816.87767.e6

Merriam, P. A., Tellez, T. L., Rosal, M. C., Olendzki, B. C., Ma, Y., Pagoto, S. L., \& Ockene, I. S. (2009). Methodology of a diabetes prevention translational research project utilizing a community-academic partnership for implementation in an underserved latino community. BMC Medical Research Methodology, 9, 20. 
doi:10.1186/1471-2288-9-20

Merton, R., K., Fiske, M., \& Kendall, P. L. (1990). The focused interview: A manual of problems and procedures (2nd ed.). New York, NY: The Free Press.

Michie, S., Abraham, C., Whittington, C., \& McAteer, J. (2009). Effective techniques in healthy eating and physical activity interventions: A meta-regression. Health Psychology, 28, 690-701.

Michie, S., Jochelson, K., Markham, W. A., \& Bridle, C. (2009). Low-income groups and behaviour change interventions: A review of intervention content, effectiveness and theoretical frameworks. Journal of Epidemiology and Community Health, 63(8), 610-622.

Mier, N., Ory, M. G., \& Medina, A. A. (2010). Anatomy of culturally sensitive interventions promoting nutrition and exercise in Hispanics: A critical examination of existing literature. Health Promotion Practice, 11(4), 541-554. doi:10.1177/1524839908328991 Miller, W. R., \& Rose, G. S. (2009). Toward a theory of motivational interviewing. American Psychologist, 64(6), 527.

Minkler, M., Wallerstein, N., \& Wilson, N. (2002). Improving health through community organization and community building. In K. Glanz, B. K. Rimer \& F. M. lewis (Eds.), Health behavior and health education: Theory, research and practice (3rd ed., pp. 279-311). San Francisco, CA: Jossey-Bass.

Minkler, M. (2005). Community-based research partnerships: Challenges and opportunities. Journal of Urban Health, 82, ii3-ii12.

Morgan, D. L. (1988). Focus groups as qualitative research. Sage University paper series on qualitative research methods. Newbury Park, CA: Sage Publications. 
Nathan, D. M., Davidson, M. B., DeFronzo, R. A., Heine, R. J., Henry, R. R., Pratley, R., ... American Diabetes Association. (2007). Impaired fasting glucose and impaired glucose tolerance: Implications for care. Diabetes Care, 30(3), 753-759. doi: $10.2337 / \mathrm{dc} 07-9920$

National Institute of Diabetes and Digestive and Kidney Disorders. (2011). National diabetes statistics, 2011 - National diabetes information clearinghouse. Retrieved from http://diabetes.niddk.nih.gov/dm/pubs/statistics/index.aspx\#Diagnosed20 on May 12, 2015.

Neighbors, C. J., Marquez, D. X., \& Marcus, B. H. (2008). Leisure-time physical activity disparities among hispanic subgroups in the united states. American Journal of Public Health, 98(8), 1460-1464. doi:AJPH.2006.096982

Nichols, G. A., Hillier, T. A., \& Brown, J. B. (2007). Progression from newly acquired impaired fasting glusose to type 2 diabetes. Diabetes Care, 30(2), 228-233. doi: $10.2337 / \mathrm{dc} 06-1392$

Norris, S. L., Zhang, X., Avenell, A., Gregg, E., Bowman, B., Schmid, C. H., \& Lau, J. (2005). Long-term effectiveness of weight-loss interventions in adults with prediabetes: A review. American Journal of Preventive Medicine, 28(1), 126-139.

O’Brien, M. J., Perez, A., Alos, V. A., Whitaker, R. C., Ciolino, J. D., Mohr, D. C., \& Ackermann, R. T. (2015). The feasibility, acceptability, and preliminary effectiveness of a promotora-led diabetes prevention program (PL-DPP) in Latinas A pilot study. The Diabetes Educator, , 0145721715586576.

O'Brien, M. J., Shuman, S. J., Barrios, D. M., Alos, V. A., \& Whitaker, R. C. (2014). A qualitative study of acculturation and diabetes risk among urban immigrant 
Latinas: Implications for diabetes prevention efforts. The Diabetes Educator, 40(5), 616-625. doi:10.1177/0145721714535992

Ockene, I. S., Tellez, T. L., Rosal, M. C., Reed, G. W., Mordes, J., Merriam, P. A., . . Ma, Y. (2012). Outcomes of a Latino community-based intervention for the prevention of diabetes: The Lawrence Latino diabetes prevention project. American Journal of Public Health, 102(2), 336-342. doi:10.2105/AJPH.2011.300357

Ogden, C. L., \& Carroll, M. D. (2010). Prevalence of overweight, obesity, and extreme obesity among adults: United States, trends 1960-1962 through 20072008. National Center for Health Statistics, 6, 1-6.

Oliverio, S. (2012). Thumbs up! Living well with diabetes. Boston, MA: Institute for Education on Health and Research.

Oza-Frank, R., \& Cunningham, S. A. (2010). The weight of US residence among immigrants: A systematic review. Obesity Reviews, 11(4), 271-280.

Pan, X. R., Li, G. W., Hu, Y. H., Wang, J. X., Yang, W. Y., An, Z. X., . . Howard, B. V. (1997). Effects of diet and exercise in preventing NIDDM in people with impaired glucose tolerance. The Da Qing IGT and diabetes study. Diabetes Care, 20(4), 537-544.

Parikh, P., Simon, E. P., Fei, K., Looker, H., Goytia, C., \& Horowitz, C. R. (2010). Results of a pilot diabetes prevention intervention in East Harlem, NewYork City: Project HEED. American Journal of Public Health, 100 Suppl 1, S232-9. doi:10.2105/AJPH.2009.170910

Parsons, M. L., \& Cornett, P. A. (2011). Sustaining the pivotal organizational 
outcome: Magnet recognition. Journal of Nursing Management, 19(2), 277-286.

Patton, M. Q. (2002). Qualitative research and evaluation methods (3rd ed.). Thousand Oaks, CA: Sage.

Perez-Escamilla, R. (2011). Acculturation, nutrition, and health disparities in Latinos. The American Journal of Clinical Nutrition, 93(5), 1163S-7S. doi:10.3945/ajen.110.003467

Perez-Escamilla, R., \& Putnik, P. (2007). The role of acculturation in nutrition, lifestyle, and incidence of type 2 diabetes among Latinos. The Journal of Nutrition, 137(4), 860-870.

Perreault, L., Kahn, S. E., Christophi, C. A., Knowler, W. C., Hamman, R. F., \& Diabetes Prevention Program Research Group. (2009). Regression from prediabetes to normal glucose regulation in the diabetes prevention program. Diabetes Care, 32(9), 1583-1588. doi:10.2337/dc09-0523

Peters, M., \& Robinson, V. (1984). The origins and status of action research. The Journal of Applied Behavioral Science, 20(2), 113-124.

Phillips, D. C. (1990). Postpositivistic science: Myths and realities. In E.G. Guba (Ed.), The Paradigm Dialog. (pp. 31-45). Thousand Oakes, CA: Sage.

Piatt, G. A., Seidel, M. C., Chen, H. Y., Powell, R. O., \& Zgibor, J. C. (2012). Twoyear results of translating the diabetes prevention program into an urban, underserved community. The Diabetes Educator, doi:10.1177/0145721712458834

Piatt, G. A., Seidel, M. C., Powell, R. O., \& Zgibor, J. C. (2013). Comparative effectiveness of lifestyle intervention efforts in the community: Results of the rethinking eating and ACTivity (REACT) study. Diabetes Care, 36(2), 202-209. 
doi: $10.2337 / \mathrm{dc} 12-0824$

Polit, D. F., \& Beck, C. T. (2008). Nursing research: Generating and assessing evidence for nursing practice (8th ed.). Philadelphia: Lippincot Williams \& Wilkins.

Prochaska, J., Redding, C., \& Evers, K. (2002). The transtheoretical model and stages of change. In K. Glanz, B.K. Rimer, \& \& F.M. Lewis, FM (Eds.), Health Behavior and Health Education: Theory, Research, and Practice (3rd ed.; pp.99120).San Francisco: Jossey-Bass.

Pronk, N. P., Boucher, J., Jeffery, R. W., Sherwood, N. E., \& Boyle, R. (2004). Reducing the incidence of type 2 diabetes mellitus. Disease Management \& Health Outcomes, 12(4), 249-258.

Ramachandran, A., Snehalatha, C., Mary, S., Mukesh, B., Bhaskar, A., \& Vijay, V. (2006). The Indian diabetes prevention programme shows that lifestyle modification and metformin prevent type 2 diabetes in Asian Indian subjects with impaired glucose tolerance (IDPP-1). Diabetologia, 49(2), 289-297.

Ramirez, A. G., Chalela, P., Gallion, K., \& Velez, L. F. (2007). Energy balance feasibility study for Latinas in Texas: A qualitative assessment. Preventing Chronic Disease, 4(4), A98

Resnicow, K., Braithwaite, R., Dilorio, C., \& Glanz, K. (2002). Applying theory to culturally diverse and unique populations. In K. Glanz, B.K. Rimer, \& \& F.M. Lewis, FM (Eds.),Health Behavior and Health Education: Theory, Research, and Practice (3rd ed.; pp. 485-509).San Francisco: Jossey-Bass.

Resnicow, K., McCarty, F., \& Baranowski, T. (2003). Are precontemplators less 
likely to change their dietary behavior? A prospective analysis. Health Education Research, 18(6), 693-705.

Resnicow, K., Soler, R., Braithwaite, R. L., Ahluwalia, J. S., \& Butler, J. (2000). Cultural sensitivity in substance use prevention. Journal of Community Psychology, 28(3), 271-290.

Rollnick, S., Miller, W. R., \& Butler, C. (2008). Motivational interviewing in health care: Helping patients change behavior. New York: Guilford Press.

Rosal, M. C., Borg, A., Bodenlos, J. S., Tellez, T., \& Ockene, I. S. (2011). Awareness of diabetes risk factors and prevention strategies among a sample of low-income Latinos with no known diagnosis of diabetes. The Diabetes Educator, 37(1), 4755. doi:10.1177/0145721710392247

Rosal, M. C., Benjamin, E. M., Pekow, P. S., Lemon, S. C., \& von Goeler, D. (2008). Opportunities and challenges for diabetes prevention at two community health centers. Diabetes Care, 31(2), 247-254. doi:10.2337/dc07-0746

Rosal, M. C., Goins, K. V., Carbone, E. T., \& Cortes, D. E. (2004). Views and preferences of low-literate Hispanics regarding diabetes education: Results of formative research Health Education \& Behavior : The Official Publication of the Society for Public Health Education, 31(3), 388-405. doi: $10.1177 / 1090198104263360$

Roy, L. (2014). Baccalaureate nursing students' perceptions of simulation and the development of clinical judgement, Widener University School of Nursing. Rubin, H. J., \& Rubin, I. S. (2005). Qualitative interviewing: The art of hearing data (2nd ed.). Thousand Oaks, CA: Sage. 
Ruggiero, L., Castillo, A., Quinn, L., \& Hochwert, M. (2012). Translation of the diabetes prevention program's lifestyle intervention: Role of community health workers. Current Diabetes Reports, 12(2), 127-137.

Ruggiero, L., Oros, S., \& Choi, Y. K. (2011). Community-based translation of the diabetes prevention program's lifestyle intervention in an underserved Latino population. The Diabetes Educator, 37(4), 564-572. doi: $10.1177 / 0145721711411107$

Rycroft-Malone, J., Seers, K., Titchen, A., Harvey, G., Kitson, A., \& McCormack, B. (2004). What counts as evidence in evidence-based practice? Journal of Advanced Nursing, 47(1), 81-90.

Sallis, J. F., Owen, N., \& Fisher, E. B. (2008). Ecological models of health behavior. Health Behavior and Health Education: Theory, Research, and Practice, 4, 465486.

Sandelowski, M. (1995). Qualitative analysis: What it is and how to begin. Research in Nursing \& Health, 18(4), 371-375.

Santoyo-Olsson, J., Cabrera, J., Freyre, R., Grossman, M., Alvarez, N., Mathur, D., . . . Stewart, A. L. (2011). An innovative multiphased strategy to recruit underserved adults into a randomized trial of a community-based diabetes risk reduction program. The Gerontologist, 51 Suppl 1, S82-93. doi:10.1093/geront/gnr026

Sartor, G., Scherstén, B., Carlström, S., Melander, A., Nordén, Å, \& Persson, G. (1980). Ten-year follow-up of subjects with impaired glucose tolerance:

Prevention of diabetes by tolbutamide and diet regulation. Diabetes, 29(1), 41-49. doi:10.2337/diab.29.1.41 
Satterfield, D. W., Volansky, M., Caspersen, C. J., Engelgau, M. M., Bowman, B. A., Gregg, E. W., . . V Vinicor, F. (2003). Community-based lifestyle interventions to prevent type 2 diabetes. Diabetes Care, 26(9), 2643-2652.

Schneider, A. L., Williams, E. K., Brancati, F. L., Blecker, S., Coresh, J., \& Selvin, E. (2013). Diabetes and risk of fracture-related hospitalization: The atherosclerosis risk in communities study. Diabetes Care, 36(5), 1153-1158. doi:10.2337/dc121168

Schwartz, R. M. (1982). Cognitive-behavior modification: A conceptual review. Clinical Psychology Review, 2(3), 267-293.

Seeman, T., Epel, E., Gruenewald, T., Karlamangla, A., \& McEwen, B. S. (2010). Socio-economic differentials in peripheral biology: Cumulative allostatic load. Annals of the New York Academy of Sciences, 1186(1), 223-239.

Seidel, M. C., Powell, R. O., Zgibor, J. C., Siminerio, L. M., \& Piatt, G. A. (2008). Translating the diabetes prevention program into an urban medically underserved community: A nonrandomized prospective intervention study. Diabetes Care, 31(4), 684-689. doi:10.2337/dc07-1869

Seo, D., \& Sa, J. (2008). A meta-analysis of psycho-behavioral obesity interventions among US multiethnic and minority adults. Preventive Medicine, 47(6), 573-582.

Shadish, W. R. (1993). Critical multiplism: A research strategy and its attendant tactics. New Directions for Program Evaluation, 1993(60), 13- 57. doi:10.1002/ev.1660

Shaw, J. E., Sicree, R. A., \& Zimmet, P. Z. (2010). Global estimates of the prevalence of diabetes for 2010 and 2030. Diabetes Research and Clinical Practice, 87(1), 4- 
14.

Skinner, B. F. (1963). Operant behavior. American Psychologist, 18(8), 503.

Skinner, B. F. (1977). Why I am not a cognitive psychologist. Behaviorism, , 5(2) 110.

Stokols, D. (1992). Establishing and maintaining healthy environments: Toward a social ecology of health promotion. American Psychologist, 47(1), 6.

Stokols, D. (1996). Translating social ecological theory into guidelines for community health promotion. American Journal of Health Promotion, 10(4), 282-298.

Tervalon, M., \& Murray-Garcia, J. (1998). Cultural humility versus cultural competence: A critical distinction in defining physician training outcomes in multicultural education. Journal of Health Care for the Poor and Underserved, $9(2), 117-125$.

Tesch, R. (1990). Qualitative research: Analysis types and software tools. New York, NY: The Falmer Press.

Torgerson, J. S., Hauptman, J., Boldrin, M. N., \& Sjostrom, L. (2004). XENical in the prevention of diabetes in obese subjects (XENDOS) study: A randomized study of orlistat as an adjunct to lifestyle changes for the prevention of type 2 diabetes in obese patients. Diabetes Care, 27(1), 155-161.

Tuomilehto, J., Lindström, J., Eriksson, J. G., Valle, T. T., Hämäläinen, H., IlanneParikka, P., .. . Uusitupa, M. (2001). Prevention of type 2 diabetes mellitus by changes in lifestyle among subjects with impaired glucose tolerance. New England Journal of Medicine, 344(18), 1343-1350. doi:10.1056/NEJM200105033441801

U.S. Department of Health and Human Services, Agency for Healthcare Research and 
Quality (AHRQ). (2013). National healthcare disparities report. (No. 14-0006).

Rockville,MD: AHRQ.

US Census Bureau. (2010). The next four decades the older population in the United States: 2010 to 2050 .Retrieved from http://www.census.gov.revproxy.brown.edu/prod/2010pubs/p25-1138.pdf

Vadheim, L. M., Brewer, K. A., Kassner, D. R., Vanderwood, K. K., Hall, T. O., Butcher, M. K., ... Harwell, T. S. (2010). Effectiveness of a lifestyle intervention program among persons at high risk for cardiovascular disease and diabetes in a rural community. The Journal of Rural Health, 26(3), 266-272.

Venditti, E. M., \& Kramer, M. K. (2012). Necessary components for lifestyle modification interventions to reduce diabetes risk. Current Diabetes Reports, 12(2), 138-146. doi:10.1007/s11892-012-0256-9

Venditti, E. M., \& Kramer, M. K. (2013). Diabetes prevention program community outreach. American Journal of Preventive Medicine, 44(4), S339- S345. doi:10.1016/j.amepre.2012.12.014

Vincent, D., Clark, L., Zimmer, L. M., \& Sanchez, J. (2006). Using focus groups to develop a culturally competent diabetes self-management program for Mexican Americans. The Diabetes Educator, 32(1), 89-97. doi:32/1/89

Wallerstein, N., \& Duran, B. (2003). The conceptual, historical, and practice roots of community based participatory research and related participatory traditions.In Israel, B.A., E. Eng, A. Schulz, E.A. Parker, \& D. Satcher (Eds.), Methods in Community-Based Participatory Research for Health, (pp. 27-52). San Francisco: Jossey-Bass. 
Wallerstein, N. B., \& Duran, B. (2006). Using community-based participatory research to address health disparities. Health Promotion Practice, 7(3), 312-323. doi:1524839906289376

Watson, J. B. (1913). Psychology as the behaviorist views it. Psychological Review, $20(2), 158$.

Whittemore, R. (2011). A systematic review of the translational research on the diabetes prevention program. Translational Behavioral Medicine, 1, 480-491. doi:10.1007/s13142-011-0062-y

Whittemore, R., Chase, S. K., \& Mandle, C. L. (2001). Validity in qualitative research. Qualitative Health Research, 11(4), 522-537.

Whittemore, R., Rosenberg, A., \& Jeon, S. (2013). A diabetes prevention program provided by home care nurses to residents of public housing communities. Journal of Health Disparities Research \& Practice, 6(1), 14-29. Retrieved from http://search.ebscohost.com/login.aspx?direct=true \&db=a9h\&AN=91578669\&site $=$ ehost-live \&scope $=$ site

Williams, I. C., Utz, S. W., Hinton, I., Yan, G., Jones, R., \& Reid, K. (2014). Enhancing diabetes self-care among rural african americans with diabetes: Results of a two-year culturally tailored intervention. The Diabetes Educator, 40(2), 231239. doi:10.1177/0145721713520570

Wing, R. R., \& Phelan, S. (2005). Long-term weight loss maintenance. The American Journal of Clinical Nutrition, 82(1 Suppl), 222S-225S.

World Health Organization. (1985). Diabetes mellitus: Report of a WHO study group. ( No. 727). Geneva: World Health Organization 
Yamaoka, K., \& Tango, T. (2005). Efficacy of lifestyle education to prevent type 2 diabetes: A meta-analysis of randomized controlled trials. Diabetes Care, 28(11), $2780-2786$.

Yoon, U., Kwok, L. L., \& Magkidis, A. (2013). Efficacy of lifestyle interventions in reducing diabetes incidence in patients with impaired glucose tolerance: A systematic review of randomized controlled trials. Metabolism, 62(2), 303-314. doi:http://dx.doi.org/10.1016/j.metabol.2012.07.009

Zhang, Q., Wang, Y., \& Huang, E. S. (2009). Changes in racial/ethnic disparities in the prevalence of type 2 diabetes by obesity level among US adults. Ethnicity \& Health, 14(5), 439-457. doi:10.1080/13557850802699155 